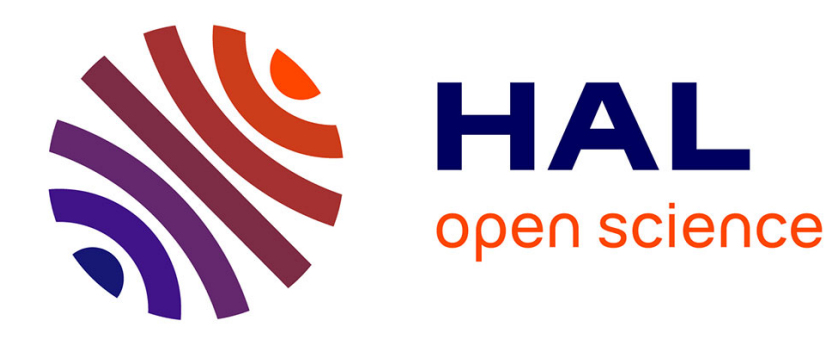

\title{
Simulating expansions without expansions
}

R. Di Cosmo, D. Kesner

\section{To cite this version:}

R. Di Cosmo, D. Kesner. Simulating expansions without expansions. RR-1911, INRIA. 1993. inria00074762

\section{HAL Id: inria-00074762 \\ https://hal.inria.fr/inria-00074762}

Submitted on 24 May 2006

HAL is a multi-disciplinary open access archive for the deposit and dissemination of scientific research documents, whether they are published or not. The documents may come from teaching and research institutions in France or abroad, or from public or private research centers.
L'archive ouverte pluridisciplinaire HAL, est destinée au dépôt et à la diffusion de documents scientifiques de niveau recherche, publiés ou non, émanant des établissements d'enseignement et de recherche français ou étrangers, des laboratoires publics ou privés. 


\title{
Simulating expansions without expansions
}

\author{
Roberto Di COSMO \\ Delia KESNER
}

\section{$N^{\circ} 1911$}

Mai 1993

PROGRAMME 2

Calcul symbolique, programmation et génie logiciel

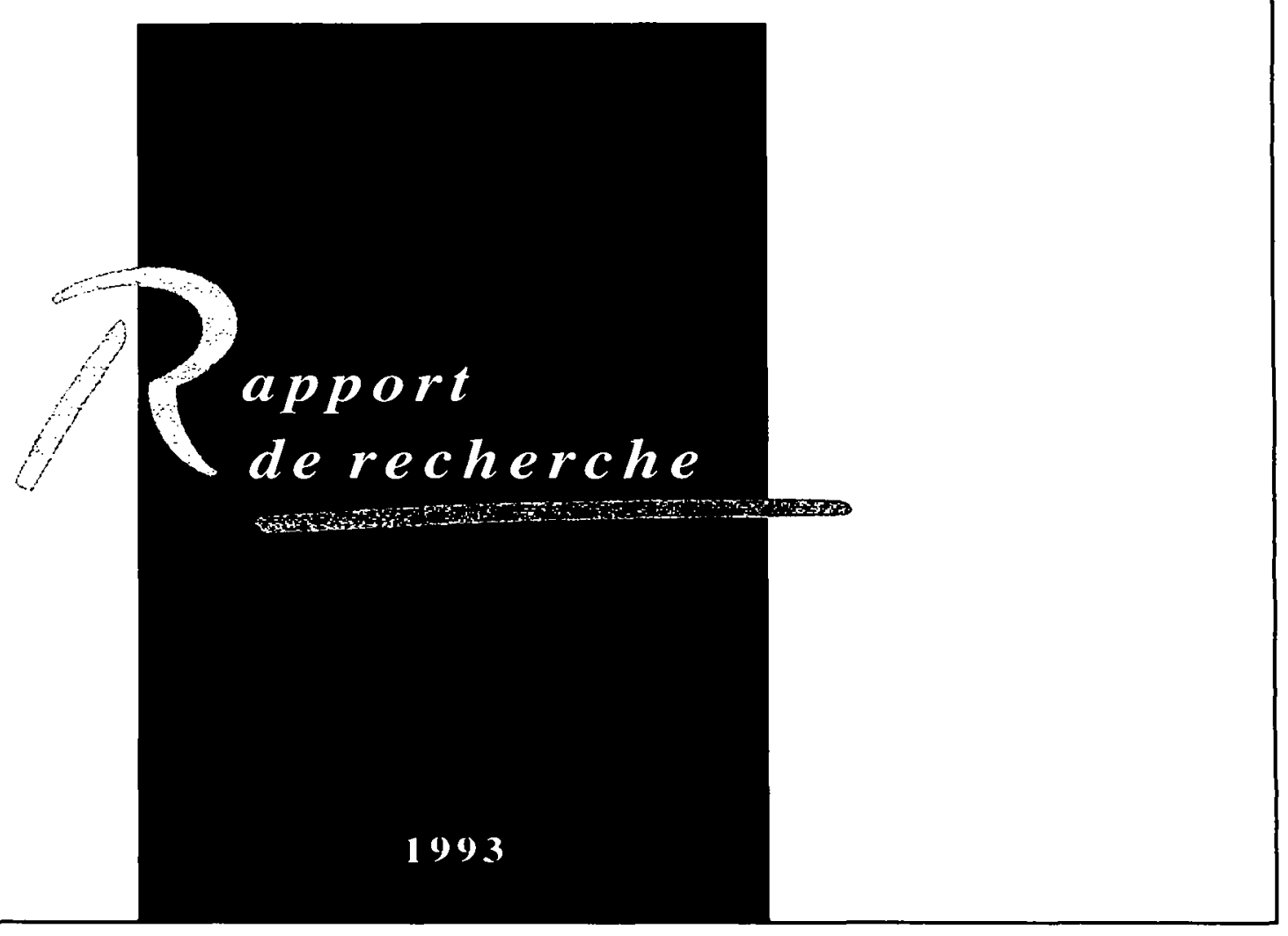




\title{
Simulating expansions without expansions
}

\section{A propos de la simulation des expansions sans expansions}

\author{
Roberto Di Cosmo* Delia Kesner ${ }^{\S}$
}

\begin{abstract}
We add extensional equalities for the functional and product types to the typed $\lambda$-calculus with not only products and terminal object, but also sums and bounded recursion (a version of recursion that does not allow recursive calls of infinite length). We provide a confluent and strongly normalizing (thus decidable) rewriting system for the calculus, that stays confluent when allowing unbounded recursion. For that, we turn the extensional equalities into expansion rules, and not into contractions as is done traditionally. We first prove the calculus to be weakly confluent, which is a more complex and interesting task than for the usual $\lambda$-calculus. Then we provide an effective mechanism to simulate expansions without expansion rules, so that the strong normalization of the calculus can be derived from that of the underlying, traditional, non extensional system. These results give us the confluence of the full calculus, but we also show how to deduce confluence directly form our simulation technique, without the weak confluence property.
\end{abstract}

\section{Résumé}

On ajoute des égalités extensionnelles pour les types flèche et produit au $\lambda$-calcul typé avec produits, sommes, objet terminal et récursivité limitée (une forme de récursivité qui ne permet pas des appels récursifs de longueur infinie). On fournit un système de réécriture confluent et fortement normalisable (donc décidable), qui reste confluent quand l'on permet la récursivité illimitée. Pour cela, on transforme les égalités extensionnelles en règles d'expansion, interprétation différente de la traditionnelle qui les oriente comme des contractions. On prouve d'abord la confluence faible du calcul, ce qui est plus difficile et intéressant que pour le $\lambda$-calcul usuel. Ensuite, on donne une méthode effective pour simuler les expansions sans les règles d'expansions, de telle façon que la normalisation forte du calcul soit réduite à celle du système non extensionnel traditionnel sousjacent. Ces résultats ainsi obtenus nous permettent de dériver la confluence du calcul entier, mais on montre aussi comment déduire directement la propriété de confluence à partir de la technique de simulation, sans avoir recours à la confluence faible.

-DMI-LIENS (CNRS URA 1347) Ecole Normale Supérieure - 45, Rue d'Ulm - 75230 Paris France

E-mail:dicosmoedmi.ens.fr

SINRIA Rocquencourt - Domaine de Voluceau, BP 105 - 78153 Le Chesnay Cedex, France and CNRS and LRI - Bât 490, Université de Pariś-Sud - 91405 Orsay Cedex, France

E-mail:kesneremargaux. inxia.fr 


\section{Contents}

1 Introduction $\quad 2$

2 Survey 2

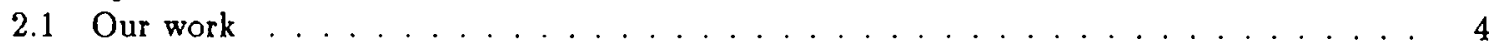

3 The Calculus $\quad \mathbf{4}$

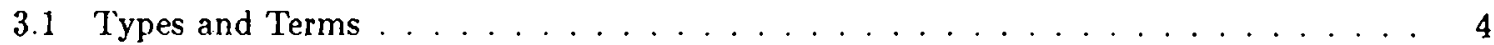

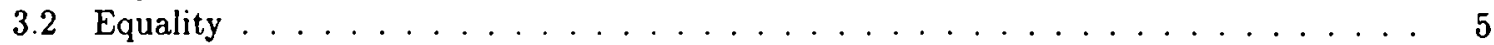

3.3 The confluent rewriting system . . . . . . . . . . . . . . . . . . 6

3.4 Influential Positions . . . . . . . . . . . . . . . . . . . . . . . 7

3.5 Adequacy of expansions for extensional equalities . . . . . . . . . . . . . 8

3.6 Basic Properties of the Calculus . . . . . . . . . . . . . . . . . . . . . . 9

4 Weak Confluence $\quad 11$

4.1 Some difficulties . . . . . . . . . . . . . . . . . . . . . . . 11

4.2 Solving Critical Pairs . . . . . . . . . . . . . . . . . . . . . . . . . . . 11

4.3 From Solved Critical Pairs to Full Weak Confluence . . . . . . . . . . . . . . . 25

5 Strong Normalization $\quad \mathbf{2 8}$

5.1 Simulating Expansions without Expansions . . . . . . . . . . . . . . . 28

5.2 Strong Normalization of the Full Calculus . . . . . . . . . . . . . . . . . . . 43

6 Strong Normalization via stability $\quad 44$

6.1 Stability ............................... . . . . . . . . . . . . . . . . . . . . . .

6.2 Properties of stability . . . . . . . . . . . . . . . . . . . . . . 45

6.3 Products, sums and basic recursion . . . . . . . . . . . . . . . . . 46

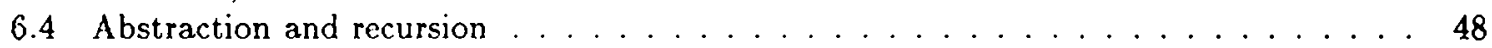

7 Strong Normalization via reducibility $\quad 50$

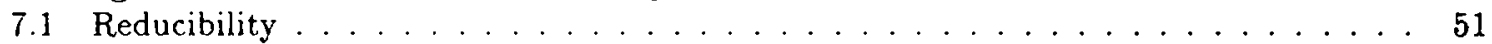

7.2 Properties of reducibility . . . . . . . . . . . . . . . . . . 51

7.2 .1 Atomic types . . . . . . . . . . . . . . . . . . 51

7.2 .2 Product types. . . . . . . . . . . . . . . . . . 52

7.2 .3 Arrow types . . . . . . . . . . . . . . . . . 52

7.2 .4 Sum types .......................... 52

7.3 Reducibility theorem . . . . . . . . . . . . . . . . . . . . 53

8 Confluence of the Full Calculus $\quad 56$

9 Adding weak extensionality for the sum type $\quad 57$

$\begin{array}{lr}10 \text { Conclusion and Future Work } & 58\end{array}$ 


\section{Introduction}

Over the past years there has been a growing interest in the properties of $\lambda$-calculus extended with various different type constructors, in particular pairs and sums, used to represent common data types. For these type constructors it is customary to provide a set of equalities that are then turned into computation rules: this is the case, for example, of the elimination rules for pairs:

$$
\left(\pi_{1}\right) \pi_{1}(\langle M, N\rangle)=M\left(\pi_{2}\right) \pi_{2}(\langle M, N\rangle)=N
$$

'They tell us how to operationally compute with objects of these types: if we have a pair $\langle M, N\rangle$, then we can decompose it to access its first or second component.

There is anyway something else that one likes to do with $\lambda$-calculus, besides using $\lambda$-terms as programs to be computed: one would like to reason about programs, to prove that they enjoy certain properties. Here is where extensional equalities come into play. In the case of functions, for example, since the only operational way to use a function is to apply it to an argument, we do not really want to consider a term $M$ of function type different from the term $\lambda x . M x$ where $x$ does not occur free in $M$ : both terms, when applied to an argument $N$, give the same result $M N$. Similarly for pairs, the only operational way to use a pair is by projecting out the first or the second component, so we do not want to consider a term $M$ of product type different from the term $\left\langle\pi_{1}(M), \pi_{2}(M)\right\rangle$ : the result of accessing any of these two terms via a first or second projection is the same term $\pi_{1}(M)$ or $\pi_{2}(M)$.

These facts can be incorporated in the calculus in the form of equalities, that one can read in at least two different ways:

- an operational way: these equalities just state possible optimizations of a program. Since a term $\left\langle\pi_{1}(M), \pi_{2}(M)\right\rangle$ is more complex then $M$, but behaves the same way, it is convenient to replace all its occurrences by $M$, as this transformation will yield an equivalent, but more efficient and smaller program. Similarly, we will replace every occurrence of $\lambda x . M x$ by $M$.

- a theoretical way: these equalities state a relation between a program and its type. They just tell us that whenever a term $M$ has a functional type, then it must really be a function, built by $\lambda$-abstraction, so we ought to replace it by $\lambda x \cdot M x$ if it is not already a function. Similarly, a term $M$ of product type has to be really a pair, built via the pair constructor, or otherwise it must be replaced by $\left\langle\pi_{1}(M), \pi_{2}(M)\right\rangle$.

As we will briefly see in the Survey, a lot of research activity has focused on the operational reading of these equalities in the tradition of $\lambda$-calculus, while only a little on the theoretical one. In this paper we will show how this last reading of the equalities provides a confluent and strongly normalizing reduction system for the simply typed $\lambda$-calculus with pairs, sums, unit type (or terminal object) and a bounded recursion operator. We also show that the same reduction system stays confluent when allowing unbounded recursion, while of course loosing the strong normalization property.

\section{Survey}

Due to the deep connections between $\lambda$-calculus, proof theory and category theory, works on extensional equalities have appeared with different motivations in all these fields.

By far, the best known extensional equality is the $\eta$ axiom that we informally introduced above, written in the $\lambda$-calculus formalism as

$$
\text { ( } \eta) \quad \lambda x . M x=M \quad \text { provided } x \text { is not free in } M
$$

This axiom, also known as extcnsionality, has traditionally been turned into a reduction, carrying the same name, by orienting the equality from left to right, interpreting operationally equality as a contraction. Such an interpretation is well behaved as it preserves confluence [CF58].

In the early 70's, the attention was focusing on products and the extensional rule for pairs, called surjective pairing, which is the analog for product types of the usual $\eta$ extensional rule.

$$
(S P) \quad\left\langle\pi_{1}(M), \pi_{2}(M)\right\rangle=M
$$


With the previous experience of the $\eta$ rule, it is easy to understand how, at that time, most of the people thought that the right way to turn such an equality into a rewrite rule was also from left to right, as a contraction. But in 1980, J.W. Klop discovered [Klo80] that, if added to the usual confluent rewrite rules for pure $\lambda$-calculus, this interpretation of $S P$ breaks confluence ${ }^{1}$.

Anyway, this first negative result was shortly after mitigated in [Pot81] for the simply typed $\lambda$ calculus with $\eta$ and SP contractions, by providing a first proof of confluence and strong normalization, later on simplified in different ways (see [Tro86] or [GLT90], for example). From then on, the contraction rule for SP was not considered harmful in a typed framework, until the seminal work by Lambek and Scott [LS86]. There, the decision problem of the equational theory of Cartesian Closed Categories (ccc's) is solved using a particular typed $\lambda$-calculus equipped with not only $\eta$ and SP equalities, but also with a special type $\mathbf{T}$ representing the tcrminal object of the ccc's ${ }^{2}$. This distinguished atomic type comes with a further extensional axiom asserting that there is exactly one term $*$ of type $\mathbf{T}$ :

$$
\text { (Top) } \quad M: \mathbf{T}=*
$$

Now, the type $\mathbf{T}$ has the bad property of destroying confluence, if the extensional equalities $\eta$ and SP are turned into contraction rules: the following are the critical pairs that arise immediately, as first pointed out by Obtulowicz, (see [LS86]):

$$
\begin{aligned}
& \left\langle\pi_{1}(x), \pi_{2}(x)\right\rangle \Rightarrow_{S P} x \quad\left\langle\pi_{1}(x), \pi_{2}(x)\right\rangle \Rightarrow_{s P} x \\
& \Downarrow^{T \text { Top }} \quad \Downarrow^{\text {Top }} \\
& \left\langle *, \pi_{2}(x)\right\rangle \quad\left\langle\pi_{1}(x), *\right\rangle \\
& (\lambda x: \mathbf{T} \cdot M x): \mathbf{T} \rightarrow A \Rightarrow_{\eta} M \quad(\lambda x: A \cdot M x): A \rightarrow \mathbf{T} \Rightarrow_{\eta} M \\
& \Downarrow_{T o p} \\
& (\lambda x: \mathbf{T} . M *): \mathbf{T} \rightarrow A \quad(\lambda x: A . *): A \rightarrow \mathbf{T}
\end{aligned}
$$

It is indeed possible, but not easy, to extend the contractive reduction system in order to recover confluence. A first step towards such a confluent system was taken by Poigné and Voss, who were not inspired by category theory, but by the implementation of algebraic data types [PV87]. In their paper, they study a calculus that includes $\lambda^{1} \beta \eta \pi *$, and notice that to solve the previous critical pairs one needs to add an infinite number of reduction rules (that can be anyway finitely described). Then confluence of such an extended system can be proved by showing weak confluence and strong normalization. Unfortunately, the critical pair for $(\lambda x: A . M x): \mathbf{T} \rightarrow A$ is missing there, and the strong normalization proof is incomplete.

More recently, Curien and the first author got interested in a polymorphic extension of $\lambda^{1} \beta \eta \pi *$, that arose in the study of the theory of object oriented programming and of isomorphisms of types [CDC91]. They give a complete (infinite) set of reduction rules for the calculus, which is proved confluent using just weak confluence, weak normalization and some additional properties.

Meanwhile, in the field of proof theory, Prawitz was suggesting [Pra71] to turn these extensional equalities into expansion rules, rather than contractions. Building on such ideas, but motivated by the study of coherence problems in category theory, Mints gives a first faulty proof that in the typed framework expansion rules, if handled with care, are weakly normalizing and preserve confluence of the typed calculus [Min79] ${ }^{3}$.

This idea of using expansion rules seems to have passed unnoticed for a long time, even if the so called $\eta$-long normal forms were well known and used in the study of higher order unification problems [Hue76]: only in these last years there has been a renewed interest in expansion rules. In recent work [Jay92], still motivated by category theoretic investigation, Jay explores a simply typed

\footnotetext{
${ }^{1}$ See [Bar81], p. 403-409 for a short history and references.

${ }^{2}$ This is the Unit type in languages like ML.

${ }^{3}$ The same ides is present in [Min77].
} 
$\lambda$-calculus with just $\mathbf{T}$ and a natural number type $\mathbf{N}$ as base types, equipped with an induction combinator for terms of type $\mathbf{N}$. He introduced expansion rules for $\eta$ and SP that are exactly the same as the ones originally used by Mints, and in [JG92] this calculus is proved confluent and strongly normalizing. Category theory is also the motivation of Cubric [Cub92], who repaired the bug in the original proof by Mints showing confluence and weak normalization (but not strong normalization). Other recent related works are [Dou93], who provides another proof of confluence and strong normalization, and [Aka93], where an intcresting divide-and-conquer approach is proposed to prove the same properties.

\subsection{Our work}

The present paper is inspired by all the previous works, but especially by [Jay92] and [PV87]. We use expansion rules to provide a confluent rewriting system for the typed $\lambda$-calculus with not only products and terminal object, but also sums and recursion. This result is derived from the confluence of a restricted system where recursion is bounded (recursive calls of infinite length are not allowed), which is proved to be weakly confluent and strongly normalizing.

We show that strong normalization of the full system can be reduced to that of the system without expansion rules, for which the traditional techniques can be used (we give two proofs, one following [GLT90], and the other following [Kri90]). For that purpose, we show that any one step reduction in the calculus with expansions can be simulated by a non-empty reduction sequence in the calculus without expansions. It turns out that this result is powerful enough to prove directly also the confluence property, as shown in section 8 .

Since the reduction with expansion rules is not a congruence, several fundamental properties that hold for the well known typed $\lambda$-calculi have to be reformulated in the expansionary framework in a different way as we will see in Section 4. For this reason we believe that the system with expansion rules deserves to be studied much more carefully, so we will undertake the task of proving directly wcak confluence: this will lead us to uncover many of the essential features of this reduction.

We introduce now the calculus and its reduction system in section 3 , then we investigate the key properties of the new reduction system: weak confluence (section 4) and strong normalization (section 5). In section 8 we derive the confluence property in two different ways and finally in the conclusion we discuss some further applications of our proof techniques.

An extended abstract of this work can be found in [DCK93].

\section{The Calculus}

It. is now time to introduce the calculus we will deal with in this paper. There are two versions, one with bounded recursion, and the other with unbounded recursion, that differ just in the term formation rule and in the equality rule for recursive terms. We will now introduce the calculus with bounded recursion and then describe how the unbounded version can be obtained from it.

\subsection{Types and Terms}

The set of types of our calculus contains a distinguished type constant $\mathbf{T}^{4}$, a denumerable set of atomic or base types, and is closed w.r.t. formation of function, product and sum, i.e. if $A$ and $B$ are types, then also $A \rightarrow B, A \times B$ and $A+B$ are types.

For each type $\Lambda$, we fix a denumerable set of variables of that type. We will use $x, y, z, \ldots$ to range over variables, and for a term $M$ we write $M: A$ to mean that $M$ is a term of type A.

The term formation rules of the calculus can then be presented as follows.

\footnotetext{
This stands for the terminal object in cce's or for the Unit type in languages like ML.
} 


$$
\begin{aligned}
& \overline{\Gamma \vdash *: \mathbf{T}} \\
& x_{1}: A_{1}, \ldots, x_{n}: A_{n} \vdash x_{i}: A_{i} \\
& 1 \leq i \leq n \text { and the } x_{i} \text { 's are pairwise distinct } \\
& \frac{\Gamma, x: A \vdash M: B}{\Gamma \vdash \lambda x: A . M: A \rightarrow B} \\
& \frac{\Gamma \vdash M: A \rightarrow B \quad \Gamma \vdash N: A}{\Gamma \vdash(M N): B} \\
& \frac{\Gamma \vdash M: A \quad \Gamma \vdash N: B}{\Gamma \vdash(M, N): A \times B} \\
& \Gamma \vdash M: B_{1} \times B_{2} \\
& \overline{\Gamma \vdash \pi_{i}(M): B_{i}} i=1,2 \\
& \Gamma \vdash M: B_{i} \\
& \Gamma \vdash i n_{B_{1}+B_{2}}^{i}(M): B_{1}+B_{2} \\
& i=1,2 \\
& \frac{\Gamma \vdash P: A_{1}+A_{2} \quad \Gamma \vdash M_{i}: A_{i} \rightarrow D}{\Gamma \vdash \operatorname{Case}\left(P, M_{1}, M_{2}\right): D} \\
& \Gamma, x: A \vdash M: A \\
& \Gamma \vdash(\operatorname{rec} x: A . M)^{i}: A \\
& i \geq 0
\end{aligned}
$$

We may omit types of variables in $\lambda$-abstractions when they are clear from the context writing $\lambda y . M$ instead of $\lambda y: C . M$.

Notation 3.1 (Free variables, substitutions) The set of free variables of a term $M$ will be noted $F V(M)$. It can be defined inductively as follows:

$$
\begin{array}{ll}
F V(*) & =\emptyset \\
F V(x) & =\{x\} \\
F V\left(O_{A}\right) & =F V(M) \\
F V(M N) & =F V(M)+F V(N) \\
F V(\langle M, N)) & =F V(M)+F V(N) \\
F V(\lambda x: A . M) & =F V(M)-\{x\} \\
F V\left((\operatorname{rec} x: A . M)^{i}\right) & =F V(M)-\{x\} \\
F V\left(\text { in }_{C}^{1}(M)\right) & =F V(M) \\
F V\left(i n_{C}^{2}(M)\right) & =F V(M) \\
F V\left(\pi_{1}(M)\right) & =F V(M) \\
F V\left(\pi_{2}(M)\right) & =F V(M) \\
F V(\operatorname{Casc}(P, M, N)) & =F V(P)+F V(M)+F V(N)
\end{array}
$$

We write $\left[N_{1}, \ldots, N_{n} / x_{1}, \ldots, x_{n}\right]$ (often abbreviated $[\bar{N} / \bar{x}]$ ) for the typed substitution mapping each variable $x_{i}: A_{i}$ to a term $N_{i}: A_{i}$. We write $M[\bar{N} / \bar{x}]$ for the term $M$ where each variable $x_{i}$ free in $M$ is replaced by $N_{i}$.

\subsection{Equality}

Besides the usual identification of terms up to $\alpha$ conversion (i.e. renaming of bound variables), our calculus is equipped with the following equalities between terms. 


$\begin{array}{lll}(\beta) & (\lambda x: A . M) N & =M[N / x] \\ \left(\pi_{1}\right) & \pi_{1}\left(\left\langle M_{1}, M_{2}\right\rangle\right) & =M_{1} \\ \left(\pi_{2}\right) & \pi_{2}\left(\left\langle M_{1}, M_{2}\right)\right) & =M_{2} \\ (\rho) & \text { Case }\left(i i_{C}^{1}(R), M_{1}, M_{2}\right) & =M_{1} R \\ & \text { Case }\left(i_{C}^{2}(R), M_{1}, M_{2}\right) & =M_{2} R \\ (\text { rec }) & (\text { rec } y: C . M)^{i+1} & =M\left[(\operatorname{rec} y: C \cdot M)^{i} / y\right] \\ (\eta) & \lambda x: A . M x & =M \text { if }\left\{\begin{array}{c}x \notin F V(M) \\ M: A \rightarrow B\end{array}\right. \\ (\delta) & \left\langle\pi_{1}(M), \pi_{2}(M)\right\rangle & =M \text { if } M: A \times B \\ (\text { Top }) & M & =* \text { if } M: \mathbf{T}\end{array}$

The index $i$ that is attached to each rec tcrm is a bound on the depth of the recursive calls that can originate from it. With such a bound, it is possible to insure the strong normalization of the associated reduction system.

The unbounded system is obtained from the bounded one by simply erasing all the bound indexes from the formation and equality rules (and the associated reduction rules). As we will show later, the bounded system can simulate any finite reduction of the unbounded system, and this fact will make it easy to extend the confluence result for the bounded system to the unbounded one. For simplicity, we will explicitly note the bound index only when necessary, dropping it whenever the properties we discuss hold in both systems.

\subsection{The confluent rewriting system}

The non extensional equality rules and the rule for $\mathbf{T}$ can be turned into a confluent rewriting system by orienting them from left to right, as follows

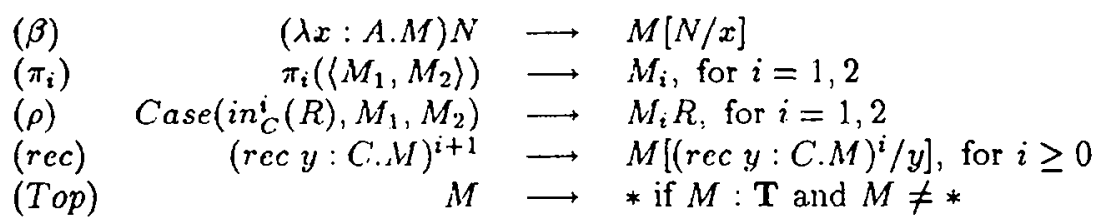

But when we want to turn the extensional equalities for functions and pairs into expansions, as explained very clearly by Jay, we must be carcful to avoid the following reduction loops:

$$
\begin{array}{llcll}
\lambda x . M & \sim & \lambda y \cdot(\lambda x \cdot M) y & \sim \lambda y \cdot M[y / x]={ }_{\alpha} \lambda x \cdot M \\
\langle M, N\rangle & \sim & \left.\sim \pi_{1}(\langle M, N\rangle), \pi_{2}(\langle M, N\rangle)\right\rangle & \sim\langle M, N\rangle \\
M N & \sim & \sim(\lambda x \cdot M x) N & \sim M N \\
\pi_{i}(P) & \sim & \pi_{i}\left(\left\langle\pi_{1}(P), \pi_{2}(P)\right\rangle\right) & \sim \pi_{i}(P)
\end{array}
$$

To break the first two loops we must disallow expansions of terms that are already $\lambda$-abstractions or pairs:

$$
\begin{aligned}
& \text { ( }) \quad M \longrightarrow \lambda x: A . M x \text { if }\left\{\begin{array}{l}
x \notin F V(M) \\
M: A \rightarrow B \text { and } M \text { is not a } \lambda \text {-abstraction }
\end{array}\right. \\
& \text { (ס) } M \longrightarrow\left\langle\pi_{1}(M), \pi_{2}(M)\right\} \text { if }\{M: A \times B \text { and } M \text { is not a pair }
\end{aligned}
$$

But this is not enough: to break the last two loops we must also forbid the $\eta$ expansion of a term in a context where this term is applied to an argument, and $\delta$ expansion of a term when such a term is the argument of a projection. This means that we cannot define the one-step reduction relation $\Longrightarrow$ on terms as the least congruence on terms containing the above reductions $\longrightarrow$, as is done usually. Instead, one defines formally $M \Longrightarrow M^{\prime}$ starting from $\longrightarrow$ by induction on the structure of the term. The definition is the same as a congruence closure but for the two last cases.

We will write $M^{\gamma_{1} \ldots, \gamma_{n}} M^{\prime}$ if $M \stackrel{\gamma_{1}}{\longrightarrow} M^{\prime}$, for some $i$ and $\stackrel{-r}{\longrightarrow}$ stands for a $\longrightarrow$ step that is not a $\gamma$ step. The one-step reduction relation between terms, denoted $\Rightarrow$ is defined as follows: 
Definition 3.2 (One-step reduction)

- If $M \longrightarrow M^{\prime}$, then $M \Longrightarrow M^{\prime}$

- If $M \Longrightarrow M^{\prime}$, then $(\operatorname{rec} x: A . M)^{i} \Longrightarrow\left(\operatorname{rec} x: A . M^{\prime}\right)^{i}$

$$
\begin{array}{lll}
\text { Case }(M, N, O) \Longrightarrow C a s e\left(M^{\prime}, N, O\right) & i n_{C}^{1}(M) \Longrightarrow \operatorname{in}_{C}^{1}\left(M^{\prime}\right) & \langle M, N\rangle \Longrightarrow\left\langle M^{\prime}, N\right\rangle \\
\operatorname{Case}(N, M, O) \Longrightarrow \operatorname{Case}(N, M, O) & i n_{C}^{2}(M) \Longrightarrow i n_{C}^{2}\left(M^{\prime}\right) & \langle N, M\rangle \Longrightarrow\left\langle N, M^{\prime}\right\rangle \\
\operatorname{Case}(N, O, M) \Longrightarrow \operatorname{Case}\left(N, O, M^{\prime}\right) & \lambda x: A . M \Longrightarrow \lambda x: A \cdot M^{\prime} & N M \Longrightarrow N M^{\prime}
\end{array}
$$

- If $M \Longrightarrow M^{\prime}$ but $M \stackrel{\rightarrow}{\longrightarrow} M^{\prime}$, then $M N \Longrightarrow M^{\prime} N$

- If $M \Longrightarrow M^{\prime}$ but $M \stackrel{-6}{\longrightarrow} M^{\prime}$, then $\pi_{i}(M) \Longrightarrow \pi_{i}\left(M^{\prime}\right)$ for $i=1,2$

Notation 3.3 The transitive and the reflexive transitive closure of $\Longrightarrow$ are noted $\Longrightarrow+$ and $\Longrightarrow$ * respectively. Similarly we define $\stackrel{\infty}{\Longrightarrow}, \stackrel{\infty}{\Longrightarrow}+$ and $\stackrel{\infty}{\Longrightarrow}$ for the unbounded system.

We will use some standard notions from the theory of rewriting system, such as redex, normal form, confluence, weak confluence, strong normalization, etc, without explicitly redefining them here.

\subsection{Influential Positions}

It is also useful to define a notion of influential positions of a term: informally, a position in a term is influential if it prevents an expansion rule from being applied at the root of the subterm found at that position. For example, $M$ occurs at an influential position in the term $M N$, as $\eta$ expansion is forbidden on $M$, no matters if it is a $\lambda$-abstraction or not. Obviously, a position in a term can be influential for $\eta$ or for $\delta$, but not for both.

Formally, we define the set $\mathcal{P}(M)$ of positions of a term $M$ and we distinguish two subsets of it: the set of influential positions for $\eta$, denoted $\mathcal{I} \mathcal{P}_{\eta}(M)$, that prevent the $\eta$ expansion rule at a subterm appearing at position $u \in \mathcal{I} \mathcal{P}_{\eta}(M)$ and the set of influential positions for $\delta$, denoted $\mathcal{I P}_{\delta}(M)$, that prevent the $\delta$ expansion rule at a subterm appearing at position $u \in \mathcal{I} \mathcal{P}_{\delta}(M)$. The concatenation of the positions $u$ and $v$ is denoted $u . v$ and the concatenation of the position $u$ with the set of positions $\mathcal{S}$ is defined as $\{u . v \mid v \in \mathcal{S}\}$. When $\mathcal{S}=\emptyset, u . \mathcal{S}=\emptyset$. Formally:

$$
\begin{aligned}
& \mathcal{P}(*) \quad=\{\epsilon\} \\
& \mathcal{P}(x) \quad=\{\epsilon\} \\
& \mathcal{P}\left(M_{1} M_{2}\right) \quad=\{\epsilon\}+1 \cdot \mathcal{P}\left(M_{1}\right)+2 \cdot \mathcal{P}\left(M_{2}\right) \\
& \mathcal{P}\left(\left\langle M_{1}, M_{2}\right\rangle\right) \quad=\{\epsilon\}+1 \mathcal{P}\left(M_{1}\right)+2 \cdot \mathcal{P}\left(M_{2}\right) \\
& \mathcal{P}(\lambda x: A . M) \quad=\{\epsilon\}+\{1\}+2 . \mathcal{P}(M) \\
& \mathcal{P}\left(\pi_{1}(M)\right) \quad=\{\epsilon\}+1 . \mathcal{P}(M) \\
& \mathcal{P}\left(\pi_{2}(M)\right)=\{\epsilon\}+1 \mathcal{P}(M) \\
& \mathcal{P}(\operatorname{Case}(P, M, N))=\{\epsilon\}+1 \mathcal{P}(P)+2 . \mathcal{P}(M)+3 \cdot \mathcal{P}(N) \\
& \mathcal{P}\left((\operatorname{rec} y: A . M)^{i}\right)=\{\epsilon\}+\{1\}+2 \cdot \mathcal{P}(M) \\
& \mathcal{P}\left(\text { in }_{C}^{1}(M)\right) \quad=\{\epsilon\}+1 \mathcal{P}(M) \\
& \mathcal{P}\left(i n_{C}^{2}(M)\right) \quad=\{\epsilon\}+1 \mathcal{P}(M) \\
& \mathcal{I P}_{\eta}(*) \quad=\emptyset \\
& \operatorname{IP}_{\eta}(x) \quad=\emptyset \\
& \operatorname{IP}_{\eta}\left(M_{1} M_{2}\right)=\{1\}+1 \cdot \mathcal{I P}_{\eta}\left(M_{1}\right)+2 . \mathcal{I P}_{\eta}\left(M_{2}\right) \\
& \mathcal{I P}_{\eta}\left(\left\langle M_{1}, M_{2}\right\rangle\right) \quad=1 . \mathcal{I} \mathcal{P}_{\eta}\left(M_{1}\right)+2 . \mathcal{I} \mathcal{P}_{\eta}\left(M_{2}\right) \\
& \mathcal{I P}_{\eta}(\lambda x: A . M) \quad=\{\epsilon\}+2 . \mathcal{I} \mathcal{P}_{\eta}(M) \\
& \mathcal{I P}_{\eta}\left(\pi_{1}(M)\right) \quad=1 . \mathcal{I} \mathcal{P}_{\eta}(M) \\
& \operatorname{IP}_{\eta}\left(\pi_{2}(M)\right) \quad=1 . \mathcal{I} \mathcal{P}_{\eta}(M) \\
& \operatorname{IP}_{\eta}(\operatorname{Case}(P, M, N))=1 . \mathcal{I P}_{\eta}(P)+2 \cdot \mathcal{I P}_{\eta}(M)+3 \cdot \mathcal{I P}_{\eta}(N) \\
& \mathcal{I P}_{\eta}\left((\text { rec } y: A . M)^{i}\right)=2 . \mathcal{I P}_{\eta}(M) \\
& \mathcal{I P}_{\eta}\left(\operatorname{in}_{C}^{1}(M)\right) \quad=1 . \mathcal{I P} \mathcal{P}_{\eta}(M) \\
& \mathcal{I P}_{\eta}\left(\operatorname{in}_{C}^{2}(M)\right) \quad=1 . \mathcal{I} \mathcal{P}_{\eta}(M)
\end{aligned}
$$




$$
\begin{aligned}
& \operatorname{IP}_{\delta}(*) \quad=0 \\
& \operatorname{IP}_{\delta}(x) \quad=\emptyset \\
& \mathcal{I P}_{\delta}\left(M_{1} M_{2}\right) \quad=1 . \mathcal{I} \mathcal{P}_{\delta}\left(M_{1}\right)+2 . \mathcal{I} \mathcal{P}_{\delta}\left(M_{2}\right) \\
& \operatorname{IP}_{\delta}\left(\left\langle M_{1}, M_{2}\right\rangle\right)=\{\epsilon\}+1 . \mathcal{I} \mathcal{P}_{\delta}\left(M_{1}\right)+2 . \mathcal{I P}_{\delta}\left(M_{2}\right) \\
& \mathcal{I P}_{\delta}(\lambda x: A . M) \quad=2 \mathcal{I P}_{\delta}(M) \\
& \mathcal{I P}_{\delta}\left(\pi_{1}(M)\right) \quad=\{1\}+1 . \mathcal{I P}_{\delta}(M) \\
& \mathcal{I P}_{\delta}\left(\pi_{2}(M)\right) \quad=\{1\}+1 \cdot \mathcal{I P}_{\delta}(M) \\
& \mathcal{I P}_{\delta}(\operatorname{Case}(P, M, N))=1 . \mathcal{I} \mathcal{P}_{\delta}(P)+2 \cdot \mathcal{I P}_{\delta}(M)+3 \cdot \operatorname{IP}_{\delta}(N) \\
& \operatorname{IP}_{\delta}\left((\operatorname{rec} y: A \cdot M)^{i}\right)=2 . \mathcal{I P}_{\delta}(M) \\
& \operatorname{IP}_{\delta}\left(\operatorname{in}_{C}^{1}(M)\right) \quad=1 . \mathcal{I P}_{\delta}(M) \\
& \mathcal{I P}_{\delta}\left(\text { in }_{C}^{2}(M)\right) \quad=1 . \mathcal{I P}_{\delta}(M)
\end{aligned}
$$

For example:

$$
\begin{aligned}
& \mathcal{P}\left(\left\langle x y,\left\langle\pi_{1}(x), z x\right\rangle\right\rangle\right)=\{\epsilon, 1,2,1.1,1.2,2.1,2.2,2.1 .1,2.2 .1,2.2 .2\} \\
& \mathcal{I} \mathcal{P}_{\eta}\left(\left\langle x y,\left\langle\pi_{1}(x), z x\right\rangle\right\rangle\right)=\{1.1,2.2 .1\} \\
& \mathcal{I} \mathcal{P}_{\delta}\left(\left\langle x y,\left\langle\pi_{1}(x), z x\right\rangle\right\rangle\right)=\{2.1 .1\}
\end{aligned}
$$

In general, we will say that $u$ is an influential position of a term $M$ if $u \in \mathcal{I P}_{\eta}(M)$ or $u \in \mathcal{I P}_{\delta}(M)$

\subsection{Adequacy of expansions for extensional equalities}

First of all, it is necessary to show that the limitations imposed on the reduction system do not make us loose any valid equality. We will show that the reduction system just introduced really generates the equalities we defined for the calculus. This comes from the fact that the limitations imposed on the reductions are introduced exactly to avoid reduction loops.

Theorem 3.4 ( $\Longrightarrow$ generates $E$ ) The equality $E$ and the reflexive, symmetric and transitive closure $R$ of $\Longrightarrow$ are the same relation.

Proof.

The fact that $R$ is included in $E$ is evident, as all the reductions rules are derived from the equality axioms by orienting and restricting them.

What we are left to show is $E \subseteq R$. It is enough to show that whenever $M=N$ comes from a single equality axiom, we can either rewrite $M$ to $N$ or $N$ to $M$ (since $R$ is reflexive, symmetric and transitive, the other cases will follow trivially).

The basic idea of the proof is to associate to each of these equality steps a reduction step in $R$. This is done in the obvious way, except in the cases that would violate one of the restrictions imposed on the expansion rules, which we will solve using exactly the reduction loop that this restriction is supposed to prevent.

Here are the problematic cases and how to deal with them. We use the usual context notation $C[M]$ to indicate a particular occurrence of a subterm $M$ of interest in the term $C[M]$.

- $C[\lambda x . M]={ }_{\eta} C[\lambda y .(\lambda x . M) y]$. We cannot associate an $\eta$ reduction to this equality, as we cannot expand something that is already an abstraction. But we can associate to it a $\beta$ reduction from $C[\lambda y \cdot(\lambda x . M) y]$ to $C[\lambda y \cdot M[y / x]]=C[\lambda x \cdot M]$.

- $\left.C[\langle M, N\rangle]=\delta\left(\pi_{1}(\langle M, N\rangle), \pi_{2}(\langle M, N\rangle)\right\rangle\right]$. We cannot expand something that is already a pair, but we can use the $\pi_{i}$ 's reduction from $\left.\left(\pi_{1}(\langle M, N\rangle), \pi_{2}(\langle M, N\rangle)\right\rangle\right]$ to $C[\langle M, N\rangle]$.

- $C[M N]={ }_{\eta} C[(\lambda x \cdot M x) N]$. Here we cannot expand $M$, which is in an influential position, but again we can use $\beta$ to go from $C[(\lambda x . M x) N]$ to $C[M N]$ (recall that $x \notin \mathrm{FV}(M))$.

- $C\left[\pi_{i}(P)\right]={ }_{\delta} C\left[\pi_{i}\left(\left\langle\pi_{1}(P), \pi_{2}(P)\right\rangle\right)\right]$. We cannot expand $P$, but we can use the $\pi_{i}$ 's to go to $C\left[\pi_{i}(P)\right]$ from $C\left[\pi_{i}\left(\left\langle\pi_{1}(P), \pi_{2}(P)\right\rangle\right)\right]$. 


\subsection{Basic Properties of the Calculus}

The following two lemmas are used to show that this calculus enjoys the subject reduction property, which guarantees that reductions preserve types.

Lemma 3.5 If $\Gamma, x: A \vdash M: C$ and $x \notin F V(M)$, then $\Gamma \vdash M: C$.

Proof. By induction on $M$.

Lemma 3.6 If $\Gamma, x: A \vdash M: C$ and $\Gamma \vdash N: A$, then $\Gamma \vdash M[N / x]: C$.

Proof. We show the property by induction on the structure of $M$

- $M \equiv *$. We have $*[N / x]=*$ and $\Gamma \vdash *: \mathbf{T}$ is an axiom.

- $M \equiv x$. We have $x[N / x]=N$ and the property trivially holds.

- $M \equiv y \not \equiv x$. We have $y[N / x]=y$ and by lemma 3.5 the property holds.

- $M \equiv \lambda y: B . P$. We have $(\lambda y: B . P)[N / x]=\lambda y: B .(P[N / x])$. Since $\Gamma, x: A \vdash \lambda y: B . P: B \rightarrow$ $D$ comes from $\Gamma, x: A, y: B \vdash P: D$ wc have by induction hypothesis $\Gamma, y: B \vdash P[N / x]: D$ and thus $\Gamma \vdash \lambda y: B .(P[N / x]): B \rightarrow D$.

- $M \equiv\left(M_{1} M_{2}\right)$. We have $\left(M_{1} M_{2}\right)[N / x]=\left(M_{1}[N / x]\right)\left(M_{2}[N / x]\right)$. Since $\Gamma, x: A \vdash\left(M_{1} M_{2}\right): C$ comes from $\Gamma, x: A \vdash M_{1}: A \rightarrow C$ and $\Gamma: x: A \vdash M_{2}: A$, by induction hypothesis $\Gamma \vdash$ $M_{1}[N / x]: A \rightarrow C$ and $\mathrm{V} \vdash M_{2}[N / x]: A$ and thus $\Gamma \vdash\left(M_{1}[N / x]\right)\left(M_{2}[N / x]\right): C$.

- $M \equiv\left\langle M_{1}, M_{2}\right\rangle$. We have $\left\{M_{1}, M_{2}\right\rangle[N / x]=\left\langle M_{1}[N / x], M_{2}[N / x]\right\rangle$. Since $\Gamma, x: A \vdash\left\langle M_{1}, M_{2}\right\rangle$ : $C_{1} \times C_{2}$ comes from $\Gamma, x: A \vdash M_{1}: C_{1}$ and $\Gamma, x: A \vdash M_{2}: C_{2}$, by induction hypothesis $\Gamma \vdash M_{1}[N / x]: C_{1}$ and $\Gamma \vdash M_{2}[N / x]: C_{2}$ and thus $\Gamma \vdash\left\langle M_{1}[N / x], M_{2}[N / x]\right\rangle: C_{1} \times C_{2}$.

- $M \equiv i n_{D_{1}+D_{2}}^{i}(P)$, for $i=1,2$. We have $i n_{D_{1}+D_{2}}^{i}(P)[N / x]=i n_{D_{1}+D_{2}}^{i}(P[N / x])$. Since $\Gamma, x$ : A $i n_{D_{1}+D_{2}}^{i}(P): D_{1}+D_{2}$ comes from $\Gamma, x: A \vdash P: D_{i}$, by induction hypothesis $\Gamma \vdash P[N / x]$ : $D_{i}$ and thus $\Gamma \vdash i n_{D_{1}+D_{2}}^{i}(P[N / x]): D_{1}+D_{2}$

- $M \equiv \pi_{i}(P)$, for $i=1,2$. We have $\pi_{i}(P)[N / x]=\pi_{i}(P[N / x])$. Since $\Gamma, x: A \vdash \pi_{i}(P): C_{i}$ comes from $\Gamma, x: A \vdash P: C_{1} \times C_{2}$, by induction hypothesis $\Gamma \vdash P[N / x]: C_{1} \times C_{2}$, and thus $\Gamma \vdash \pi_{i}(P[N / x]): C_{i}$.

- $M \equiv \operatorname{Case}\left(P, M_{1}, M_{2}\right)$. We have $\operatorname{Case}\left(P, M_{1}, M_{2}\right)[N / x]=\operatorname{Case}\left(P[N / x], M_{1}[N / x], M_{2}[N / x]\right)$. $\Gamma, x: A \vdash \operatorname{Case}\left(P, M_{1}, M_{2}\right): C$ comes from $\Gamma, x: A \vdash P: B_{1}+B_{2}, \Gamma, x: A \vdash M_{1}: B_{1} \rightarrow C$ and $\Gamma, x: A \vdash M_{2}: B_{2} \rightarrow C$. By induction hypothesis $\Gamma \vdash P[N / x]: B_{1}+B_{2}, \Gamma \vdash M_{1}[N / x]: B_{1} \rightarrow C$ and $\Gamma \vdash M_{2}[N / x]: B_{2} \rightarrow C$ and thus $\Gamma \vdash \operatorname{Case}\left(P[N / x], M_{1}[N / x], M_{2}[N / x]\right): C$.

- $M \equiv(\operatorname{rec} y: C . P)^{i}$. We have $(\operatorname{rec} y: C . P)^{i}[N / x]=(\operatorname{rec} y: C . P[N / x])^{i}$. Since $\Gamma, x: A \vdash$ $(\text { rec } y: C . P)^{i}: C$ comes from $\Gamma, x: A, y: C \vdash P: C$, by induction hypothesis $\Gamma, y: C \vdash$ $P[N / x]: C$ and thus $\mathrm{\Gamma} \vdash(\operatorname{rec} y: C . P[N / x])^{i}: C$.

Proposition 3.7 (Subject Reduction) If $\mathrm{I} \vdash R: C$ and $R \Longrightarrow R^{\prime}$, then $\Gamma \vdash R^{\prime}: C$

Proof. We proceed by cases. Let's see first the case of one external reduction step:

- $(\lambda x: A . M) N \stackrel{\beta}{\longrightarrow} M[N / x]$. As $\Gamma \vdash(\lambda x: A . M) N: C$ comes from $\Gamma, x: A \vdash M: C$ and $\Gamma \vdash N: A$, then $\Gamma \vdash M[N / x]: C$ holds by lemma 3.6.

- $M \stackrel{\eta}{\longrightarrow} \lambda x: A . M x$. As $\Gamma \vdash M: A \rightarrow D$ and $\Gamma, x: A \vdash x: A$, then $\Gamma, x: A \vdash M x: D$ and $\Gamma \vdash \lambda x: A . M x: A \rightarrow D$.

- $M \stackrel{\text { Top }}{\longrightarrow}$ and $\Gamma \vdash *: \mathbf{T}$ is an axiom.

- $\pi_{i}\left(\left\langle M_{1}, M_{2}\right\rangle\right) \stackrel{\pi_{i}}{\longrightarrow} M_{i}$. We have $\Gamma \vdash \pi_{i}\left(\left\langle M_{1}, M_{2}\right\rangle\right): C_{i}$ if $\Gamma \vdash\left\langle M_{1}, M_{2}\right\rangle: C_{1} \times C_{2}$ and this holds if $\Gamma \vdash M_{i}: C_{i}$.

- $M \stackrel{8}{\longrightarrow}\left\langle\pi_{1}(M), \pi_{2}(M)\right\rangle$. Since $\Gamma \vdash M: C_{1} \times C_{2}$, then $\Gamma \vdash \pi_{1}(M): C_{1}$ and $\Gamma \vdash \pi_{2}(M): C_{2}$ and thus $\Gamma \vdash\left\langle\pi_{1}(M), \pi_{2}(M)\right\rangle: C_{1} \times C_{2}$. 
- $\operatorname{Case}\left(i_{B_{1}+B_{2}}^{i}\left(P, M_{1}, M_{2}\right) \stackrel{\rho}{\longrightarrow} M_{i} P\right.$. Since $\Gamma \vdash \operatorname{Case}\left(i_{B_{1}+B_{2}}^{i}(P), M_{1}, M_{2}\right): C$, then $\Gamma \vdash$ $i n_{B_{1}+B_{2}}^{i}(P): B_{1}+B_{2}, \Gamma \vdash M_{1}: B_{1} \rightarrow C$ and $\Gamma \vdash M_{2}: B_{2} \rightarrow C$. Then we have $\Gamma \vdash P: B_{i}$ and $\Gamma \vdash M_{i}: B_{i} \rightarrow C$ and therefore $\Gamma \vdash M_{i} P: C$.

- $(\operatorname{rec} y: C . M)^{i+1} \stackrel{\text { rec }}{\longrightarrow} M\left[(\operatorname{rec} y: C . M)^{i} / y\right]$. Since $\Gamma \vdash(\text { rec } y: C . M)^{i+1}: C$, then $\Gamma, y: C \vdash$ $M: C$ and $\Gamma \vdash(\operatorname{rec} y: C . M)^{i}: C$. Applying lemma $3.6 \Gamma \vdash M\left[(\operatorname{rec} y: C . M)^{i} / y\right]: C$.

Now, let's see the case of one internal reduction step.

- If $M N \Longrightarrow M^{\prime} N$, where $M \Longrightarrow M^{\prime}$. We have $\Gamma \vdash M N: C$ if $\Gamma \vdash M: A \rightarrow C$ and $\Gamma \vdash N: A$ By induction hypothesis $\Gamma \vdash M^{\prime}: A \rightarrow C$ and thus $\Gamma \vdash M^{\prime} N: C$.

- If $M N \Longrightarrow M N^{\prime}$, where $N \Longrightarrow N^{\prime}$. We have $\Gamma \vdash M N: C$ if $\Gamma \vdash M: A \rightarrow C$ and $\Gamma \vdash N: A$ By induction hypothesis $\Gamma \vdash N^{\prime}: A$ and thus $\Gamma \vdash M N^{\prime}: C$.

- $\lambda x: A . M \Longrightarrow \lambda x: A . M^{\prime}$, where $M \Longrightarrow M^{\prime}$. We have $\Gamma \vdash \lambda x: A . M: A \rightarrow B$ if $\Gamma, x: A \vdash$ $M: B$. By induction hypothesis $\Gamma, x: A \vdash M^{\prime}: B$ and thus $\Gamma \vdash \lambda x: A . M^{\prime}: A \rightarrow B$.

- $\langle M, N\rangle \Longrightarrow\left\langle M^{\prime}, N\right\rangle$ if $M \Longrightarrow M^{\prime}$. We have $\Gamma \vdash\langle M, N\rangle: C_{1} \times C_{2}$ if $\Gamma \vdash M: C_{1}$ and $\Gamma \vdash N: C_{2}$. By induction hypothesis $\Gamma \vdash M^{\prime}: C_{1}$ and thus $\Gamma \vdash\left\langle M^{\prime}, N\right\rangle: C_{1} \times C_{2}$.

- $\langle M, N\rangle \Longrightarrow\left\langle M, N^{\prime}\right\rangle$ if $N \Rightarrow N^{\prime}$. We have $\Gamma \vdash\langle M, N\rangle: C_{1} \times C_{2}$ if $\Gamma \vdash M: C_{1}$ and $\Gamma \vdash N: C_{2}$. By induction hypothesis $\Gamma \vdash N^{\prime}: C_{2}$ and thus $\Gamma \vdash\left\langle M, N^{\prime}\right\rangle: C_{1} \times C_{2}$.

- $\pi_{i}(M) \Longrightarrow \pi_{i}\left(M^{\prime}\right)$, for $i=1,2$ where $M \Longrightarrow M^{\prime}$. We have $\Gamma \vdash \pi_{i}(M): C_{i}$ if $\Gamma \vdash M: C_{1} \times C_{2}$. By induction hypothesis $\Gamma \vdash M^{\prime}: C_{1} \times C_{2}$ and thus $\Gamma \vdash \pi_{i}\left(M^{\prime}\right): C_{i}$.

- $i n_{D_{1}+D_{2}}^{i}(M) \Longrightarrow i n_{D_{1}+D_{2}}^{i}\left(M^{\prime}\right)$, for $i=1,2$ where $M \Longrightarrow M^{\prime}$. We have $\Gamma \vdash i n_{D_{1}+D_{2}}^{i}(M)$ : $D_{1}+D_{2}$ if $\Gamma \vdash M: D_{i}$. By induction hypothesis $\Gamma \vdash M^{\prime}: D_{i}$ and thus $\Gamma \vdash i n_{D_{1}+D_{2}}^{i}\left(M^{\prime}\right)$ : $D_{1}+D_{2}$.

- $\operatorname{Case}(P, M, N) \Longrightarrow \operatorname{Case}\left(P^{\prime}, M, N\right)$, where $P \Rightarrow P^{\prime}$. We have $\Gamma \vdash \operatorname{Case}(P, M, N): C$ if $\Gamma \vdash P: A+B, \Gamma \vdash M: A \rightarrow C$ and $\Gamma \vdash N: B \rightarrow C$. By induction hypothesis $\Gamma \vdash P^{\prime}: A+B$ and thus $\Gamma \vdash \operatorname{Case}\left(P^{\prime}, M, N\right): C$.

- $\operatorname{Case}(P, M, N) \Longrightarrow \operatorname{Case}\left(P, M^{\prime}, N\right)$, where $M \Rightarrow M^{\prime}$. We have $\Gamma \vdash \operatorname{Case}(P, M, N): C$ if $\Gamma \vdash P: A+B, \Gamma \vdash M: A \rightarrow C$ and $\Gamma \vdash N: B \rightarrow C$. By induction hypothesis $\Gamma \vdash M^{\prime}: A \rightarrow C$ and thus $\Gamma \vdash \operatorname{Case}\left(P, M^{\prime}, N\right): C$.

- $\operatorname{Case}(P, M, N) \Longrightarrow \operatorname{Case}\left(P, M, N^{\prime}\right)$, where $N \Longrightarrow N^{\prime}$. We have $\Gamma \vdash \operatorname{Case}(P, M, N): C$ if $\Gamma \vdash P: A+B, \Gamma \vdash M: A \rightarrow C$ and $\Gamma \vdash N: B \rightarrow C$. By induction hypothesis $\Gamma \vdash N^{\prime}: B \rightarrow C$ and thus $\Gamma \vdash \operatorname{Case}\left(P, M, N^{\prime}\right): C$.

- $(\operatorname{rec} x: C . M)^{i} \Longrightarrow\left(\operatorname{rec} x: C . M^{\prime}\right)^{i}$, where $M \Longrightarrow M^{\prime}$. We have $\Gamma \vdash(\operatorname{rec} x: C . M)^{i}: C$ if $\Gamma, x: C \vdash M: C$. By induction hypothesis $\Gamma, x: C \vdash M^{\prime}: C$ and thus $\Gamma \vdash\left(\operatorname{rec} x: C . M^{\prime}\right)^{i}: C$.

Another remarquable property of this calculus can be stated as follows:

Lemma 3.8 If $M$ is in normal form w.r.t. the system without the $\eta, \delta$ and Top rules and $M \stackrel{\eta, \delta, T o p}{\longrightarrow} R$, then $R$ is in normal form w.r.t. the systcm without $\eta, \delta$ and Top.

Proof. Suppose $M$ has no $\beta, \pi_{i}, \rho$ or $r e c$ redexes. Notice first that a $\rho$ redex cannot be created in $R$ as there is no way to introduce an $i n^{i}$ expression using the $\eta, \delta$ and Top rules. The same for rec. We are left with the following two cases:

- Suppose $R$ has a $\beta$ redex. Then $R \equiv C[(\lambda x . P) N]$ and since $M$ contains no $\beta$ redexes, we have necessarily $M \equiv C[S N], P \equiv S x$ and $S \stackrel{\eta}{\longrightarrow} \lambda x . S x$. But this is not possible because $\eta$ expansions are not allowed on terms appearing in influential positions for $\eta$.

- Suppose $R$ has a $\pi_{i}$ redex. Then $R \equiv C\left[\pi_{i}(\langle M, N\rangle)\right]$ and since $M$ contains no $\pi_{i}$ 's redexes, we have necessarily $M \equiv C\left[\pi_{i}\left(T^{\prime}\right)\right], M \equiv \pi_{1}(T), N \equiv \pi_{2}(T)$ and $T \stackrel{b}{\longrightarrow}\left\langle\pi_{1}(T), \pi_{2}(T)\right\rangle$. But this is not possible because $\delta$ expansions are not allowed on terms appearing in influential positions for $\delta$. 
Corollary 3.9 If $M$ is in normal form with respect to the system without the $\eta, \delta$ and Top rules, then the $\eta-\delta-$ Top normal form of $M$ is in normal form.

\section{Weak Confluence}

In this section we set off to prove that the reduction system proposed above is actually weakly confluent, i.e. that whenever $M^{\prime} \Longleftarrow M \Longrightarrow M^{\prime \prime}$ we can find a term $M^{\prime \prime \prime}$ s.t. $M^{\prime} \Longrightarrow{ }^{*} M^{\prime \prime \prime} \Longleftarrow M^{\prime \prime}$. The proof is fairly more complex here than in the case of $\lambda$-calculus where extensional equalities are interpreted as contractions, and this is due to the fact that the reduction relation $\Longrightarrow$ introduced above is not a congruence on terms.

\subsection{Some difficulties}

In particular, in the simply typed $\lambda$-calculus whenever $M \Longrightarrow^{*} M^{\prime}$ then $\pi_{i}(M) \Longrightarrow^{*} \pi_{i}\left(M^{\prime}\right)$, and if also $N \Longrightarrow^{*} N^{\prime}$, then $M N \Longrightarrow^{*} M^{\prime} N^{\prime}$, but this is no longer true now: indeed, we have $x$ : $A \rightarrow B \Longrightarrow \lambda z: A . x z$, but $x N$ cannot reduce to $(\lambda z: A . x z) N$.

These properties still hold for those reduction sequences $M \Longrightarrow^{*} M^{\prime}$ that do not involve expansions at the root:

\section{Remark 4.1}

- Let $M \equiv M_{0} \Longrightarrow M_{1} \Longrightarrow \ldots \Longrightarrow M_{n-1} \Longrightarrow M_{n} \equiv M^{\prime}$ be a reduction sequence and let $N \Longrightarrow^{*} N^{\prime}$, where in the first reduction sequence there are no expansions applied at the root positions of the $M_{i}$ 's. Then, $M N \Longrightarrow^{*} M^{\prime} N^{\prime}$.

- Let $M \equiv M_{0} \Longrightarrow M_{1} \Longrightarrow \ldots \Longrightarrow M_{n-1} \Longrightarrow M_{n} \equiv M^{\prime}$ be a reduction sequence where none of the $M_{i}^{\prime} s$ is expanded at the root. Then $\pi_{i}(M) \Longrightarrow^{*} \pi_{i}\left(M^{\prime}\right)$, for $i=1,2$.

Proof.

- Take the reduction $M \equiv M_{0} \Longrightarrow M_{1} \Longrightarrow \ldots \Longrightarrow M_{n-1} \Longrightarrow M_{n} \equiv M^{\prime}$ : since there are no $\eta$ expansions at the root we can form the reduction sequence $M N \Rightarrow M_{1} N \Rightarrow \ldots \Longrightarrow$ $M_{n-1} N \Longrightarrow M_{n} N \equiv M^{\prime} N$. Then, we take the sequence $N \Rightarrow N_{1} \Rightarrow \ldots \Longrightarrow N_{p-1} \Rightarrow N_{p} \equiv$ $N^{\prime}$ and we propagate it on the right of $M^{\prime}$, i.e, $M^{\prime} N \Longrightarrow M^{\prime} N_{1} \Longrightarrow \ldots \Longrightarrow M^{\prime} N_{p-1} \Longrightarrow$ $M^{\prime} N_{p} \equiv M^{\prime} N^{\prime}$.

- Take the reduction $M \Longrightarrow M_{1} \Longrightarrow \ldots \Longrightarrow M_{n-1} \Longrightarrow M_{n} \equiv M^{\prime}$. Since there are no $\delta$ expansions at the root, we can form the reduction sequence $\pi_{i}(M) \Longrightarrow \pi_{i}\left(M_{1}\right) \Longrightarrow \ldots \Longrightarrow$ $\pi_{i}\left(M_{n-1}\right) \Longrightarrow \pi_{i}\left(M_{n}\right) \equiv \pi_{i}\left(M^{\prime}\right)$.

$\square$

\subsection{Solving Critical Pairs}

In this calculus, it is no longer true that reduction is stable by substitution, as in the traditional $\lambda$-calculus:

\section{Remark 4.2}

If $P \Longrightarrow P^{\prime}, N \Longrightarrow N^{\prime}$, it is not truc in gencral that $P[N / x] \Longrightarrow^{*} P^{\prime}[N / x]$ and $P[N / x] \Longrightarrow{ }^{*} P\left[N^{\prime} / x\right]$.

Indecd, $x: A \rightarrow B \Longrightarrow \lambda z: A . x z$, but $x[\lambda y: A . w / x]=\lambda y: A . w$ cannot reduce in our system to $\lambda z: A \cdot(\lambda y: A . w) z=\lambda z: A \cdot x z[\lambda y: A . w / x]$, and $(y M)[x / y]=x M$ cannot reduce to $(\lambda z: A . x z) M=$ $(y M)[\lambda z: A . x z / y]$.

We can prove some weaker properties: if $P \Longrightarrow P^{\prime}$, then $P[N / x]$ and $P^{\prime}[N / x]$ have a common reduct (Lenma 4.5), and similarly $P[N / x]$ and $P\left[N^{\prime} / x\right]$ when $N \Longrightarrow N^{\prime}$ (Lemma 4.6). This suffices for our purpose of proving weak confluence of the reduction system.

First of all it is useful to recall here a basic property of substitutions that do hold in our calculus. 
Lemma 4.3 If $x \not \equiv y$ and $x \notin F V(L)$, then

$$
M[N / x][L / y]=M[L / y][N[L / y] / x]
$$

Lemma 4.4 If $P^{n, \Delta, T_{0} p} P^{\prime}$, then $P[N / x] \Longrightarrow^{*} P^{\prime}[N / x]$ or $P^{\prime}[N / x] \Longrightarrow^{*} P[N / x]$. Moreover, if the expansion does not takc place at the root of $P$, then there are no expansions at root positions in the sequences $P[N / x] \Longrightarrow^{*} P^{\prime}[N / x]$ and $P^{\prime}[N / x] \Longrightarrow^{*} P[N / x]$.

Proof.

- $P \stackrel{n}{\longrightarrow} \lambda z . P z$. Then $P$ is not a $\lambda$-abstraction, $P^{\prime}[N / x]=\lambda z . P[N / x] z$ and there are two possible cases:

- If $P[N / x]$ is not a $\lambda$-abstraction, $P[N / x] \stackrel{\eta}{\longrightarrow} \lambda z \cdot P[N / x] z$ since $P$ is of type $\rightarrow$ and so $P[N / x]$ is also of type $\rightarrow$ by lemma 3.6 .

- If $P[N / x]$ is a $\lambda$-abstraction, then $P \equiv x, N \equiv \lambda y \cdot N^{\prime}$ and:

$(\lambda z \cdot x z)\left[\lambda y \cdot N^{\prime} / x\right]=\lambda z \cdot\left(\lambda y \cdot N^{\prime}\right) z \stackrel{B}{\longrightarrow} \lambda z \cdot\left(N^{\prime}[z / y]\right)={ }_{\alpha} \lambda y \cdot N^{\prime}=x\left[\lambda y \cdot N^{\prime} / x\right]$.

- $P \stackrel{\delta}{\longrightarrow}\left\langle\pi_{1}(P), \pi_{2}(P)\right\rangle$. Then $P$ is not a pair, $P^{\prime}[N / x]=\left\langle\pi_{1}(P[N / x]), \pi_{2}(P[N / x])\right\rangle$ and there are two possible cases:

- If $P[N / x]$ is not a Pair, $P[N / x] \stackrel{6}{\longrightarrow}\left(\pi_{1}(P[N / x]), \pi_{2}(P[N / x])\right)$ since $P$ is of type $\times$ and so $P[N / x]$ is also of type $x$ by lemma 3.6.

- If $P[N / x]$ is a pair, then $P \equiv x$ and $N \equiv\left\langle N_{1}, N_{2}\right\rangle$ and:

$$
\begin{array}{ll}
\left\langle\pi_{1}(x), \pi_{2}(x)\right\rangle\left[\left\langle N_{1}, N_{2}\right\rangle / x\right] & x\left[\left\langle N_{1}, N_{2}\right\rangle / x\right] \\
= & = \\
\left\langle\pi_{1}\left(\left\langle N_{1}, N_{2}\right\rangle\right), \pi_{2}\left(\left\langle N_{1}, N_{2}\right\rangle\right)\right\rangle \stackrel{\pi_{1}}{\Longrightarrow}\left\langle N_{1}, \pi_{2}\left(\left\langle N_{1}, N_{2}\right\rangle\right)\right\rangle \stackrel{\pi_{2}}{\Longrightarrow} & \left\langle N_{1}, N_{2}\right\rangle
\end{array}
$$

- $P \stackrel{\text { Top }}{\longrightarrow}$ *. Then $P[N / x] \stackrel{\text { Top }}{\longrightarrow} *=*[N / x]$ since $P$ is of type $\mathbf{T}$ and so $P[N / x]$ is also of type $\mathbf{T}$ by lemma 3.6.

Using the previous Lemma, we can precisely describe the interaction between reductions and substitutions.

\section{Lemma 4.5 (Substitution Lemma (i))}

If $P \Longrightarrow P^{\prime}$, then $P[N / x] \Longrightarrow^{*} P^{\prime}[N / x]$ or $P^{\prime}[N / x] \Longrightarrow^{*} P[N / x]$. Moreover, if no expansion take place at the root position of $P$, then there are no expansions at root positions in the reduction sequences $P[N / x] \Longrightarrow P^{\prime}[N / x]$ and $P^{\prime}[N / x] \Longrightarrow^{*} P[N / x]$.

Proof. We show the property by induction on the structure of $P$.

1. $P \equiv *$. The term * is in normal form and there is no possible reduction.

2. $P \equiv y$. In this case $P^{\eta, 8, T_{0}, p} P^{\prime}$ and by lemma 4.4 the property holds.

3. $P \equiv \lambda y . Q$. Since $P \stackrel{\eta}{\longrightarrow} P^{\prime}$ is not possible because $P$ is a $\lambda$-abstraction,$P \stackrel{6}{\longrightarrow} P^{\prime}$ is neither possible because $P$ is not of type $\times$, and $P \stackrel{T_{r p}}{\longrightarrow} *$ is neither possible since $P$ is not of type $\mathrm{T}$, we have $P^{\prime} \equiv \lambda y \cdot Q^{\prime}$, where $Q \Longrightarrow Q^{\prime}$. By i.h. $Q[N / x] \Longrightarrow^{*} Q^{\prime}[N / x]$ or $Q^{\prime}[N / x] \Longrightarrow^{*} Q[N / x]$.

If the first case,

$$
(\lambda y \cdot Q)[N / x]=\lambda y \cdot(Q[N / x]) \Longrightarrow^{*} \lambda y \cdot\left(Q^{\prime}[N / x]\right)=\left(\lambda y \cdot Q^{\prime}\right)[N / x]
$$

In the second case,

$$
\left(\lambda y \cdot Q^{\prime}\right)[N / x]=\lambda y \cdot\left(Q^{\prime}[N / x]\right) \Longrightarrow^{*} \lambda y \cdot(Q[N / x])=(\lambda y \cdot Q)[N / x]
$$


4. $P \equiv P_{1} P_{2}$. There are several cases to consider:

- If $P^{\eta, \delta, T_{0} p} P^{\prime}$ the property holds by lemma 4.4 .

- If $P \equiv\left(\lambda z . P_{3}\right) P_{2}$, then $\left(\lambda z . P_{3}\right) P_{2} \stackrel{g}{\rightarrow} P_{3}[N / x]$ and

$$
\left(\lambda z . P_{3}\right) P_{2}[N / x]=\left(\lambda z . P_{3}[N / x]\right) P_{2}[N / x] \stackrel{\beta}{\longrightarrow} P_{3}[N / x]\left[P_{2}[N / x] / z\right]=\text { lemma 4.3 } P_{3}\left[P_{2} / z\right][N / x]
$$

- If $P^{\prime} \equiv P_{1}^{\prime} P_{2}$, where $P_{1} \Longrightarrow P_{1}^{\prime}$. By i.h. $P_{1}[N / x] \Longrightarrow^{*} P_{1}^{\prime}[N / x]$ or $P_{1}^{\prime}[N / x] \Longrightarrow^{*} P_{1}[N / x]$. Suppose the first case holds. Since $P_{1} P_{2} \Longrightarrow P_{1}^{\prime} P_{2}$, then $P_{1} \stackrel{\eta}{\rightarrow} P_{1}^{\prime}$ is not possible and by lemma 4.4 there are no $\eta$ expansions at the root positions of the terms appearing in $P_{1}[N / x] \Longrightarrow P_{1}^{\prime}[N / x]$. We have

$$
\left(P_{1} P_{2}\right)[N / x]=P_{1}[N / x] P_{2}[N / x] \Longrightarrow^{*} P_{1}^{\prime}[N / x] P_{2}[N / x]=\left(P_{1}^{\prime} P_{2}\right)[N / x]
$$

In the same way the property holds for the case $P_{1}^{\prime}[N / x] \Longrightarrow{ }^{*} P_{1}[N / x]$.

- If $P^{\prime} \equiv P_{1} P_{2}^{\prime}$, where $P_{2} \Longrightarrow P_{2}^{\prime}$. By i.h. $P_{2}[N / x] \Longrightarrow{ }^{*} P_{2}^{\prime}[N / x]$ or $P_{2}^{\prime}[N / x] \Longrightarrow^{*} P_{2}[N / x]$. Suppose the first case holds, then

$$
\left(P_{1} P_{2}\right)[N / x]=P_{1}[N / x] P_{2}[N / x] \Longrightarrow * P_{1}[N / x] P_{2}^{\prime}[N / x]=\left(P_{1} P_{2}^{\prime}\right)[N / x]
$$

In the same way the property holds for the case $P_{2}^{\prime}[N / x] \Longrightarrow^{*} P_{2}[N / x]$.

5. $P \equiv i n_{C_{1}+C_{2}}^{i}(Q)$. Since $i n_{C_{1}+C_{2}}^{i}(Q)$ is of type $C_{1}+C_{2}$, then $P^{\text {n.6.Top }} P^{\prime}$ is not possible. Therefore $P^{\prime} \equiv$ in $_{C_{1}+C_{2}}\left(Q^{\prime}\right)$, where $Q \Longrightarrow Q^{\prime}$ and by induction hypothesis $Q[N / x] \Longrightarrow^{*} Q^{\prime}[N / x]$ or $Q^{\prime}[N / x] \Longrightarrow{ }^{*} Q[N / x]$.

If the first case,

$$
i n_{C_{1}+C_{2}}^{i}(Q)[N / x]=i n_{C_{1}+C_{2}}^{i}(Q[N / x]) \Longrightarrow^{*} i n_{C_{1}+C_{2}}^{i}\left(Q^{\prime}[N / x]\right)=i n_{C_{1}+C_{2}}^{i}\left(Q^{\prime}\right)[N / x]
$$

In the second

$$
i n_{C_{1}+C_{2}}^{i}\left(Q^{\prime}\right)[N / x]=i n_{C_{1}+C_{2}}^{i}\left(Q^{\prime}[N / x]\right) \Longrightarrow \Rightarrow^{*} i_{C_{1}+C_{2}}^{i}(Q[N / x])=i n_{C_{1}+C_{2}}^{i}(Q)[N / x]
$$

6. $P \equiv\left\langle P_{1}, P_{2}\right\rangle$. Since $P \stackrel{\delta}{\longrightarrow} P^{\prime}$ is not possible because $P$ is a pair, $P \stackrel{\eta}{\longrightarrow} P^{\prime}$ is neither possible because $P$ is not of type $\rightarrow$, and $P \stackrel{T_{o r}}{\longrightarrow} P^{\prime}$ is neither possible because $P$ is not of type $\mathbf{T}$, we have $P^{\prime} \equiv\left\langle P_{1}^{\prime}, P_{2}\right\rangle$, where $P_{1} \Longrightarrow P_{1}^{\prime}$ or $P^{\prime} \equiv\left\langle P_{1}, P_{2}^{\prime}\right\rangle$, where $P_{2} \Longrightarrow P_{2}^{\prime}$.

In the first case $P_{1}[N / x] \Longrightarrow^{*} P_{1}^{\prime}[N / x]$ or $P_{1}^{\prime}[N / x] \Longrightarrow^{*} P_{1}[N / x]$ hold by induction hypothesis and then either

$$
\left\langle P_{1}, P_{2}\right\rangle[N / x]=\left\langle P_{1}[N / x], P_{2}[N / x]\right\rangle \Longrightarrow^{*}\left\langle P_{1}^{\prime}[N / x], P_{2}[N / x]\right\rangle=\left\langle P_{1}^{\prime}, P_{2}\right\rangle[N / x]
$$

or

$$
\left\langle P_{1}^{\prime}, P_{2}\right\rangle[N / x] \Longrightarrow^{*}\left\langle P_{1}, P_{2}\right\rangle[N / x]
$$

In the second case $P_{2}[N / x] \Longrightarrow{ }^{*} P_{2}^{\prime}[N / x]$ or $P_{2}^{\prime}[N / x] \Longrightarrow^{*} P_{2}[N / x]$ hold by induction hypothesis and then

$$
\left\langle P_{1}, P_{2}\right\rangle[N / x]=\left\langle P_{1}[N / x], P_{2}[N / x]\right\rangle \Longrightarrow^{*}\left\langle P_{1}[N / x], P_{2}^{\prime}[N / x]\right\rangle=\left\langle P_{1}, P_{2}^{\prime}\right\rangle[N / x]
$$

or

$$
\left\langle P_{1}, P_{2}^{\prime}\right\rangle[N / x] \Longrightarrow^{*}\left(P_{1}, P_{2}^{\prime}\right\rangle[N / x]
$$


7. $P \equiv \pi_{i}(Q)$. If $\stackrel{P^{\eta, \delta, T o p}}{\longrightarrow} P^{\prime}$ the property holds by lemma 4.4. If not, $P^{\prime} \equiv \pi_{i}\left(Q^{\prime}\right)$, where $Q \Longrightarrow Q^{\prime}$ and by induction hypothesis $Q[N / x] \Rightarrow^{*} Q^{\prime}[N / x]$ or $Q^{\prime}[N / x] \Rightarrow^{*} Q[N / x]$. Since $\pi_{i}(Q) \Longrightarrow \pi_{i}\left(Q^{\prime}\right)$, then $Q \longrightarrow Q^{\prime}$ is not a $\delta$-expansion and thus there are no $\delta$-expansions appearing at the root positions of the terms in $Q[N / x] \Longrightarrow^{*} Q^{\prime}[N / x]$ and $Q^{\prime}[N / x] \Longrightarrow^{*} Q[N / x]$ (first and second case resp.). Therefore either

$$
\pi_{i}(Q)[N / x]=\pi_{i}(Q[N / x]) \Longrightarrow^{*} \pi_{i}\left(Q^{\prime}[N / x]\right)=\pi_{i}\left(Q^{\prime}\right)[N / x]
$$

or

$$
\pi_{i}\left(Q^{\prime}\right)[N / x] \Longrightarrow^{*} \pi_{i}(Q)[N / x]
$$

8. $P \equiv \operatorname{Case}\left(Q, M_{1}, M_{2}\right)$. If $\stackrel{n, \text {, , Top }}{\longrightarrow} P^{\prime}$ the property holds by lemma 4.4 . If not, there are five possibilities:

- $Q \equiv i n_{B_{1}+B_{2}}^{i}(R)$ and $C a s e\left(i n_{B_{1}+B_{2}}^{i}(R), M_{1}, M_{2}\right) \stackrel{\rho}{\Rightarrow} * M_{i} R$. Since

$$
\operatorname{Case}\left(\operatorname{in}_{B_{2}+B_{2}}^{i}(R), M_{1}, M_{2}\right)[N / x]=\operatorname{Case}\left(\operatorname{in}_{B_{1}+B_{2}}^{i}(R[N / x]), M_{1}[N / x], M_{2}[N / x]\right)
$$

and this last term reduces by a $p$-rule to $M_{i}[N / x] R[N / x]=\left(M_{i} R\right)[N / x]$ the property holds.

- $P^{\prime} \equiv \operatorname{Case}\left(Q^{\prime}, M_{1}, M_{2}\right)$, where $Q \Longrightarrow Q^{\prime}$. By induction hypothesis $Q[N / x] \Longrightarrow{ }^{*} Q^{\prime}[N / x]$ or $Q^{\prime}[N / x] \Longrightarrow * Q[N / x]$.

In the first case

$$
\begin{array}{cc}
\operatorname{Case}\left(Q, M_{1}, M_{2}\right)[N / x] & \operatorname{Case}\left(Q^{\prime}, M_{1}, M_{2}\right)[N / x] \\
= & = \\
\operatorname{Case}\left(Q[N / x], M_{1}[N / x], M_{2}[N / x]\right) \Longrightarrow * & \operatorname{Case}\left(Q^{\prime}[N / x], M_{1}[N / x], M_{2}[N / x]\right)
\end{array}
$$

In the second case $\operatorname{Case}\left(Q^{\prime}, M_{1}, M_{2}\right)[N / x] \Longrightarrow^{*} \operatorname{Case}\left(Q, M_{1}, M_{2}\right)[N / x]$.

- $P^{\prime} \equiv \operatorname{Case}\left(Q, M_{1}^{\prime}, M_{2}\right)$, where $M_{1} \Longrightarrow M_{1}^{\prime}$. By induction hypothesis $M_{1}[N / x] \Longrightarrow^{*} M_{1}^{\prime}[N / x]$ or $M_{1}^{\prime}[N / x] \Longrightarrow{ }^{*} M_{1}[N / x]$.

In the first case

$$
\begin{aligned}
& \operatorname{Case}\left(Q, M_{1}, M_{2}\right)[N / x] \quad \operatorname{Case}\left(Q, M_{1}^{\prime}, M_{2}\right)[N / x] \\
& \operatorname{Case}\left(Q[N / x], M_{1}[N / x], M_{2}[N / x]\right) \Longrightarrow * \quad \operatorname{Case}\left(Q[N / x], M_{1}^{\prime}[N / x], M_{2}[N / x]\right)
\end{aligned}
$$

In the second case $\operatorname{Case}\left(Q, M_{1}^{\prime}, M_{2}\right)[N / x] \Longrightarrow^{*} \operatorname{Case}\left(Q, M_{1}, M_{2}\right)[N / x]$.

- $P^{\prime} \equiv \operatorname{Case}\left(Q, M_{1}, M_{2}^{\prime}\right)$, where $M_{2} \Longrightarrow M_{2}^{\prime}$. By induction hypothesis $M_{2}[N / x] \Longrightarrow{ }^{*} M_{2}^{\prime}[N / x]$ or $M_{2}^{\prime}[N / x] \Longrightarrow{ }^{*} M_{2}[N / x]$.

In the first case

$$
\begin{array}{cc}
\operatorname{Case}\left(Q, M_{1}, M_{2}\right)[N / x] & \operatorname{Case}\left(Q, M_{1}, M_{2}^{\prime}\right)[N / x] \\
= & = \\
\operatorname{Case}\left(Q[N / x], M_{1}[N / x], M_{2}[N / x]\right) \Longrightarrow * & \operatorname{Case}\left(Q[N / x], M_{1}[N / x], M_{2}^{\prime}[N / x]\right)
\end{array}
$$

In the second case $\operatorname{Case}\left(Q, M_{1}, M_{2}^{\prime}\right)[N / x] \Longrightarrow^{*} \operatorname{Case}\left(Q, M_{1}, M_{2}\right)[N / x]$.

9. $P \equiv(\operatorname{rec} y: B . Q)^{i}$. If $\stackrel{\eta, \delta, T_{o p}}{\longrightarrow} P^{\prime}$ the property holds by lemma 4.4 . If not there are two cases to consider:

- $P^{\prime} \equiv Q\left[(\operatorname{rec} y: B . Q)^{i-1} / y\right]$. We have $(\operatorname{rec} y: B \cdot Q)^{i}[N / x]=(\operatorname{rec} y: B \cdot Q[N / x])^{i}$ and this last term reduces to $Q[N / x]\left[(\operatorname{rec} y: B . Q[N / x])^{i-1} / y\right]$ that is equal to $Q[($ rec $y$ : $\left.B \cdot Q)^{i-1}[N / x] / y\right][N / 2:]=Q\left[(\text { rec } y: B \cdot Q)^{i-1} / y\right][N / x]$ by lemma 4.3 . 
- $P^{\prime} \equiv\left(\operatorname{rec} y: B \cdot Q^{\prime}\right)^{i}$, where $Q \Longrightarrow Q^{\prime}$. By induction hypothesis $Q[N / x] \Longrightarrow{ }^{*} Q^{\prime}[N / x]$ or $Q^{\prime}[N / x] \Longrightarrow^{*} Q[N / x]$ and thus

$(\operatorname{rec} y: B \cdot Q)^{i}[N / x]=(\operatorname{rec} y: B \cdot Q[N / x])^{i} \Longrightarrow^{*}\left(\operatorname{rec} y: B \cdot Q^{\prime}[N / x]\right)^{i}=\left(\operatorname{rec} y: B \cdot Q^{\prime}\right)^{i}[N / x]$

or

$$
\left(\operatorname{rec} y: B \cdot Q^{\prime}\right)^{i}[N / x] \Longrightarrow^{*}(\operatorname{rec} y: B \cdot Q)^{i}[N / x]
$$

\section{Lemma 4.6 (Substitution Lemma (ii))}

If $N \stackrel{R}{\Longrightarrow} N^{\prime}$, then $M[N / x] \Longrightarrow^{*} M^{\prime \prime}{ }^{*} \Longleftarrow M\left[N^{\prime} / x\right]$ for some term $M^{\prime \prime}$. These reduction sequences contain expansions at the root only if $M \equiv x$ and $R$ is an expansion applied at the root of $N$.

Proof. We will show that $M[N / x] \stackrel{R}{\Longrightarrow} M^{\prime \prime *} \Longleftarrow M\left[N^{\prime} / x\right]$ for some term $M^{\prime \prime}$ and that these reduction sequences contain expansions at the root only if $M \equiv x$ and $R$ is an expansion applied at the root of $N$.

This is a very common lemma in the theory of $\lambda$-calculus, where the term $M^{\prime \prime}$ is always $M\left[N^{\prime} / x\right]$ and the proof is straightforward context closure of the reduction $R$. Here the conditions imposed on the expansion rules make it necessary to state the lemma this way. Effectively, the only interesting cases of the proof are the ones for application and projections, where we cannot always apply context closure for the reduction $R$, and have to make some steps backwards from $M\left[N^{\prime} / x\right]$ to $M[N / x]$.

Notice that every time the required reductions are built by context closure, there is no rule applied at the root and we state this fact here once for all. We proceed by induction on $M$ :

- $M \equiv x$

$M[N / x]=N \stackrel{R}{\Longrightarrow} N^{\prime}=M\left[N^{\prime} / x\right]$ (in this case our $M^{\prime \prime}$ is $N^{\prime}$ )

- $M \equiv y \not \equiv x$ or $M \equiv *: \mathbf{T}$

Then $M[N / x]=M=M\left[N^{\prime} / x\right]$ (in this case our $M^{\prime \prime}$ is $M$ )

- $M \equiv\left(M_{1} M_{2}\right)$

We find by induction hypothesis terms $M_{1}^{\prime \prime}$ and $M_{2}^{\prime \prime}$ such that

$$
M_{1}[N / x] \stackrel{R}{\Longrightarrow}{ }^{*} M_{1}^{\prime \prime} \Longleftarrow M_{1}\left[N^{\prime} / x\right], \text { and } M_{2}[N / x] \stackrel{n}{\Longrightarrow} * M_{2}^{\prime \prime}{ }^{*} \Longleftarrow M_{2}\left[N^{\prime} / x\right] .
$$

Here $M_{1}$ is in an influential position for $\eta$, so we have to be careful about the reductions occurring in $M_{1}[N / x] \stackrel{R}{\Longrightarrow} M_{1}^{\prime \prime} \Longleftarrow M_{1}\left[N^{\prime} / x\right]$. We have the following cases:

- If $M_{1} \not \equiv x$, or $\mathrm{R}$ is not an expansion at the root of $N$, we know by inductive hypothesis that the reductions $M_{1}[N / x] \stackrel{R}{\Longrightarrow}{ }^{*} M_{1}^{\prime \prime}{ }^{*}=M_{1}\left[N^{\prime} / x\right]$ do not contain any expansions, and in particular no $\eta$ rule, at the root position, so we can apply context closure for application and get

$$
\begin{array}{cc}
\left(M_{1} M_{2}\right)[N / x] & \left(M_{1} M_{2}\right)\left[N^{\prime} / x\right] \\
= & = \\
\left(M_{1}[N / x] M_{2}[N / x]\right) \stackrel{R}{=} *\left(M_{1}^{\prime \prime} M_{2}^{\prime \prime}\right) * & =\left(M_{1}\left[N^{\prime} / x\right] M_{2}\left[N^{\prime} / x\right]\right) .
\end{array}
$$

- If $M_{1} \equiv x$, and the expansion rule $\mathrm{R}$ is $\eta$ at the root of $N$, then $N^{\prime} \equiv \lambda z . N z$ and we can close our diagram as follows

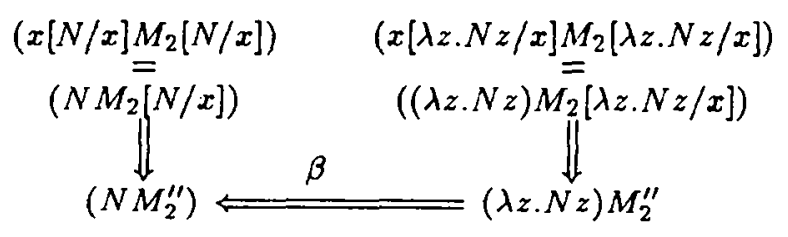


Here, the vertical reductions are built by context closure, while the horizontal one is a $\beta$, so no expansion rule is applied at the root in the overall reduction sequence.

- $M \equiv \lambda z: A . M_{1}$

If $x \neq z$, the result follows from $\left(\lambda z: A \cdot M_{1}\right)[N / x]=\lambda z: A \cdot M_{1}=\left(\lambda z: A \cdot M_{1}\right)\left[N^{\prime} / x\right]$. Otherwise, by induction hypothesis there is a term $M_{1}^{\prime \prime}$ such that $M_{1}[N / x] \stackrel{R}{\Rightarrow}{ }^{*} M_{1}^{\prime \prime} \rightleftharpoons M_{1}\left[N^{\prime} / x\right]$, so we can apply the context closure rule for abstraction and get that

$$
\begin{array}{cc}
\left(\lambda z: A \cdot M_{1}\right)[N / x] & \left(\lambda z \cdot M_{1}\right)\left[N^{\prime} / x\right] \\
= & = \\
\left(\lambda z: A \cdot M_{1}[N / x]\right) \Longrightarrow * \lambda z: A \cdot M_{1}^{\prime \prime}{ }^{*} \Longleftarrow\left(\lambda z: A \cdot M_{1}\left[N^{\prime} / x\right]\right)
\end{array}
$$

- $M \equiv \pi_{i}\left(M_{1}\right)$

We find by induction hypothesis a term $M_{1}^{\prime \prime}$ such that

$$
M_{1}[N / x] \stackrel{R}{\Longrightarrow} M_{1}^{\prime \prime} \Longleftarrow M_{1}\left[N^{\prime} / x\right] .
$$

Here $M_{1}$ is in an influential position for $\delta$, so we have to be careful about the reductions occurring in $M_{1}[N / x] \stackrel{R}{\Longrightarrow} M_{1}^{\prime \prime} \Longleftarrow M_{1}\left[N^{\prime} / x\right]$. We have the following cases:

- If $M_{1} \not \equiv x$, or $\mathrm{R}$ is not an expansion at the root of $N$, we know by inductive hypothesis that the reductions $M_{1}[N / x] \stackrel{R}{\Rightarrow}{ }^{*} M_{1}^{\prime \prime} \Leftarrow M_{1}\left[N^{\prime} / x\right]$ do not contain any expansions, and in particular no $\delta$ rule, at the root position, so we can apply context closure for projections and get

$$
\pi_{i}\left(M_{1}\right)[N / x] \stackrel{R}{\Longrightarrow} * \pi_{i}\left(M_{1}^{\prime \prime}\right) * \pi_{i}\left(M_{1}\right)\left[N^{\prime} / x\right] .
$$

- If $M_{1} \equiv x$, and the expansion rule $\mathrm{R}$ is $\delta$ at the root of $N$, then $N^{\prime} \equiv\left\langle\pi_{1}(N), \pi_{2}(N)\right\rangle$ and we can close our diagram as follows

$$
\begin{array}{ccc}
\pi_{i}(x[N / x]) & & \pi_{i}\left(x\left[\left\langle\pi_{1} N, \pi_{2} N\right\rangle / x\right]\right) \\
= & \pi & = \\
\pi_{i}(N) & \Longleftrightarrow & \pi_{i}\left(\pi_{1} N, \pi_{2} N\right)
\end{array}
$$

Here, the vertical reductions are built by context closure, while the horizontal one is a $\pi$, so no expansion rule is applied at the root in the overall reduction sequence.

- $M \equiv\left\langle M_{1}, M_{2}\right\rangle$

We find by induction hypothesis terms $M_{1}^{\prime \prime}$ and $M_{2}^{\prime \prime}$ such that $M_{1}[N / x] \stackrel{n}{\Rightarrow}{ }^{*} M_{1}^{\prime \prime}{ }^{*} \Longleftarrow M_{1}\left[N^{\prime} / x\right]$ and $M_{2}[N / x] \stackrel{R}{\Longrightarrow}{ }^{*} M_{2}^{\prime \prime}{ }^{*} \Longleftarrow M_{2}\left[N^{\prime} / x\right]$. So, we can apply the context closure rule for application and get that

$$
\begin{array}{cc}
\left(\left\langle M_{1}, M_{2}\right\rangle\right)[N / x]= & \left(\left\langle M_{1}, M_{2}\right\rangle\right)\left[N^{\prime} / x\right] \\
\left\langle M_{1}[N / x], M_{2}[N / x]\right\rangle & \left\langle M_{1}\left[N^{\prime} / x\right], M_{2}\left[N^{\prime} / x\right]\right\rangle \\
\left.* M_{1}^{\prime \prime}, M_{2}^{\prime \prime}\right\rangle & =
\end{array}
$$

- $M \equiv i n_{C}^{i}\left(M_{1}\right)$

We find by induction hypothesis a term $M_{1}^{\prime \prime}$ such that $M_{1}[N / x] \stackrel{R}{\Rightarrow}{ }^{*} M_{1}^{\prime \prime}{ }^{*} M_{1}\left[N^{\prime} / x\right]$. so we can apply the context closure rule for $i n^{i}$ and get that

$$
\begin{aligned}
& i n_{C}^{i}\left(M_{1}[N / x]\right) \quad i n_{C}^{i}\left(M_{1}\left[N^{\prime} / x\right]\right) \\
& = \\
& i n_{C}^{i}\left(M_{1}\right)[N / x] \Longrightarrow^{*} i n_{C}^{i}\left(M_{1}^{\prime \prime}\right) * i n_{C}^{i}\left(M_{1}\right)\left[N^{\prime} / x\right]
\end{aligned}
$$


- $M \equiv \operatorname{Case}\left(P, M_{1}, M_{2}\right)$

We find by induction hypothesis $P^{\prime \prime}, M_{1}^{\prime \prime}$ and $M_{2}^{\prime \prime}$ such that $P[N / x] \stackrel{R}{\Longrightarrow} * P^{\prime \prime} \approx P\left[N^{\prime} / x\right]$ and $M_{1}[N / x] \stackrel{R}{\Longrightarrow}{ }^{*} M_{1}^{\prime \prime} \rightleftarrows M_{1}\left[N^{\prime} / x\right]$ and $M_{2}[N / x] \stackrel{R}{\Longrightarrow} M_{2}^{\prime \prime *} \Longleftarrow M_{2}\left[N^{\prime} / x\right]$. So, we can apply the context closure rule for Case and get that

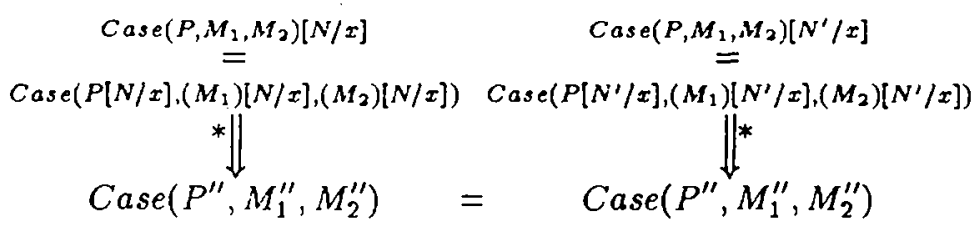

- $M \equiv\left(\operatorname{rec} z: A \cdot M_{1}\right)^{i}$

We assume $z \not \equiv x$ (otherwise the result trivially holds). We find by induction hypothesis a term $M_{1}^{\prime \prime}$ such that $M_{1}[N / x] \stackrel{R}{\Longrightarrow}{ }^{*} M_{1}^{\prime \prime}{ }^{*} \Longleftarrow M_{1}\left[N^{\prime} / x\right]$. so we can apply the context closure rule for rec and get that

$$
\begin{array}{rr}
\left(\operatorname{rec} z: A \cdot M_{1}\right)^{i}[N / x] & \left(\operatorname{rec} z: A \cdot M_{1}\right)^{i}\left[N^{\prime} / x\right] \\
= & = \\
\left(\operatorname{rec} z: A \cdot M_{1}[N / x]\right)^{i} \Longrightarrow *\left(\operatorname{rec} z: A \cdot M_{1}^{\prime \prime}\right)^{i} \Longleftarrow\left(\operatorname{rec} z: A \cdot M_{1}\left[N^{\prime} / x\right]\right)^{i}
\end{array}
$$

Example 4.7 Take $M=\langle x y, x\rangle, N=w$ and $N^{\prime}=\lambda z:$ A.wz. Then

$$
M[N / x]=\langle w y, w\rangle \Longrightarrow\langle w y, \lambda z: A . w z\rangle \Longleftarrow\langle(\lambda z: A . w z) y, \lambda z: A . w z\rangle=M\left[N^{\prime} / x\right]
$$

Looking carefully through the proof of the previous Lemma 4.6, one can see that the only cases where it is needed to apply a reverse reduction are those corresponding to an expansion rule applied at the root of $N$ and to the presence in $M$ of some free occurrences of $x$ in influential positions. So, we can also state the following

Corollary 4.8 (Reverse reductions) Let $N \stackrel{R}{\Longrightarrow} N^{\prime}$. In case $R$ is not an expansion rule applied at the root of $N$ (an external expansion rule) or $x$ does not occur at an influential position in $M$, then $M[N / x] \stackrel{R}{\Longrightarrow}{ }^{*} M\left[N^{\prime} / x\right]$

Lemma 4.5 and 4.6 suffice to prove that all critical pairs arising from a term $M$ by a $\beta$-reduction and another reduction rule can be solved. We can then state the following:

\section{Proposition 4.9 (Critical Pairs are solvable)}

If $M \rightarrow M^{\prime}$ and $M \Rightarrow M^{\prime \prime}$, then $\exists R$ such that $M^{\prime} \Longrightarrow^{*} R$ and $M^{\prime \prime} \Longrightarrow^{*} R$.

Proof. We consider every possible case of reduction from $M$ to $M^{\prime}$.

1. $M \stackrel{B}{\longrightarrow} M^{\prime}$. Thus $M \cong(\lambda x . P) N$.

1.1. If $M \Longrightarrow M^{\prime \prime}$ is internal, there are two cases:

1.1.1. $P \Longrightarrow P^{\prime}$

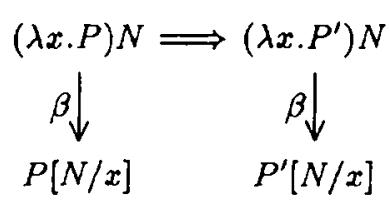

By lemma 4.5 we have $P[N / x] \Longrightarrow P^{\prime}[N / x]$ or $P^{\prime}[N / x] \Longrightarrow^{*} P[N / x]$. 
1.1.2. $N \Longrightarrow N^{\prime}$

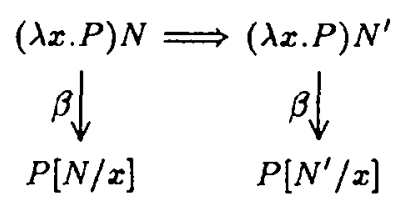

By lemma 4.6 there is a term $R$ such that $P[N / x] \Longrightarrow^{*} R$ and $P\left[N^{\prime} / x\right] \Rightarrow^{*} R$.

1.2. If $M \Rightarrow M^{\prime \prime}$ is external:

1.2.1. $M \equiv(\lambda x . P) N \stackrel{n}{\longrightarrow} \lambda y \cdot((\lambda x . P) N) y \equiv M^{\prime \prime}$

1.2.1.1. If $P[N / x]$ is not a $\lambda$-abstraction:

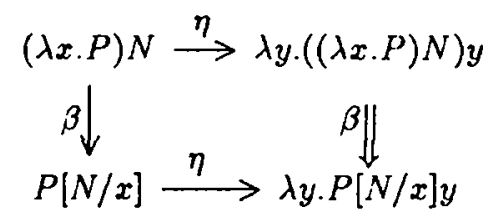

1.2.1.2. If $P[N / x]$ is a $\lambda$-abstraction we have two cases:

1.2.1.2.1. If $P$ is a $\lambda$-abstraction:

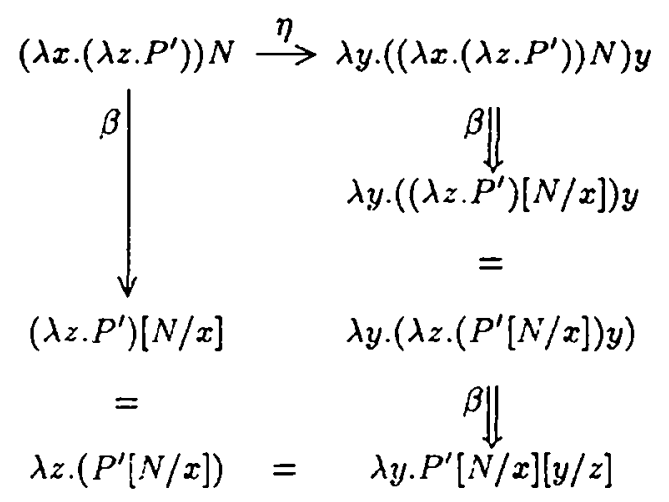

1.2.1.2.2. If $P=x$ and $N$ is a $\lambda$-abstraction $\lambda z . N^{\prime}$ :

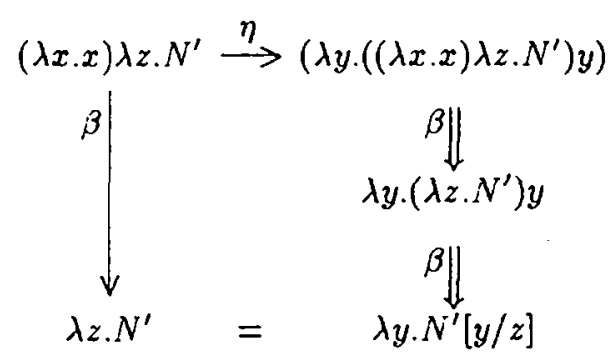

1.2.2. $M \equiv(\lambda x . P) N \stackrel{8}{\longrightarrow}\left(\pi_{1}((\lambda x . P) N), \pi_{2}((\lambda x . P) N)\right) \equiv M^{\prime \prime}$

1.2.2.1. If $P[N / x]$ is not a pair we have:

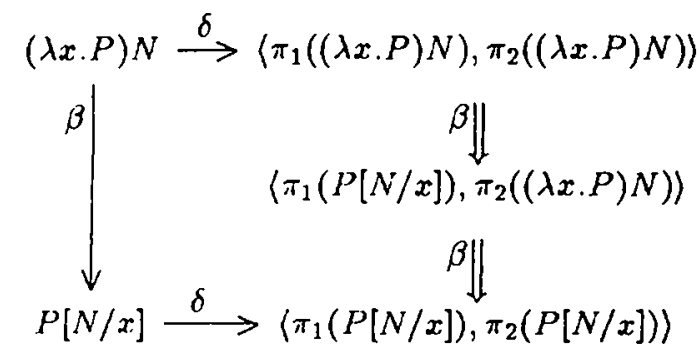


1.2.2.2. If $P[N / x]$ is a pair we have two more cases:

1.2.2.2.1. $P$ is also a pair $\left(P_{1}, P_{2}\right)$ :

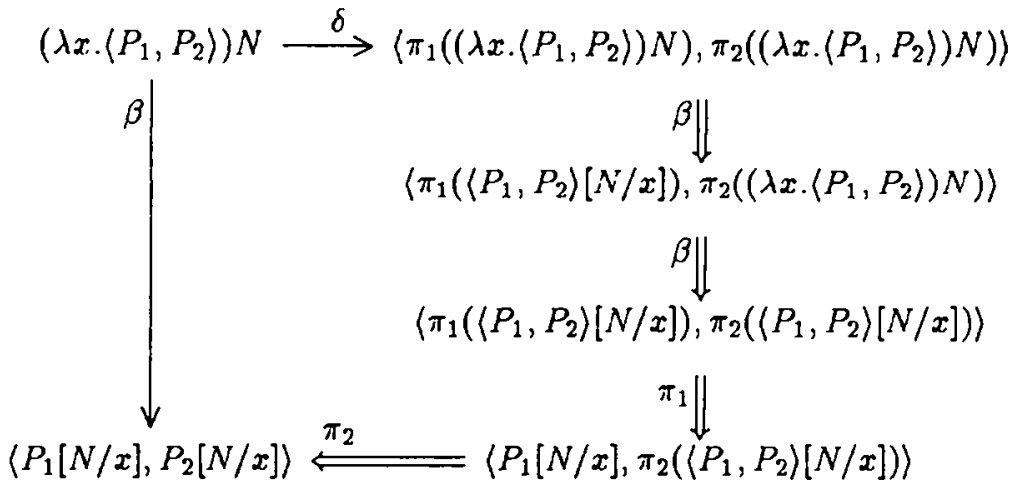

1.2.2.2.2. $P=x$ and $N$ is a pair $\left\langle N_{1}, N_{2}\right\rangle$ :

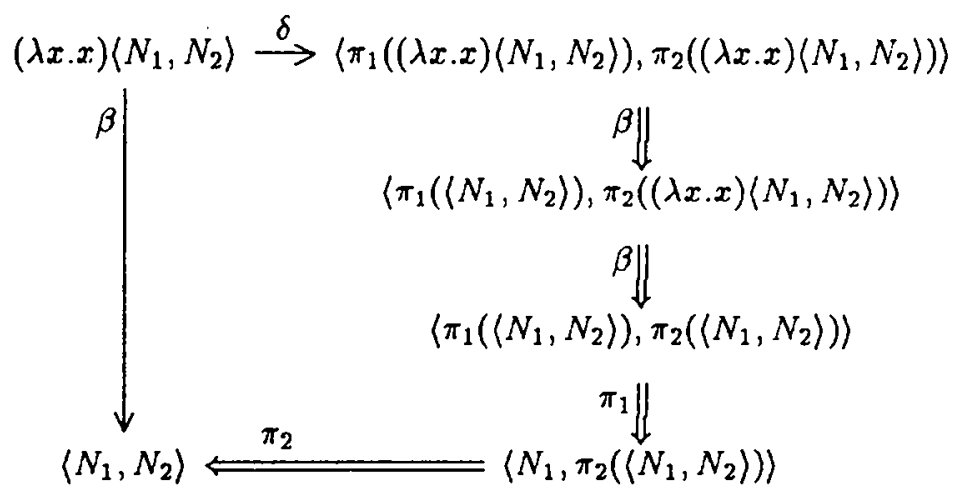

1.2.3. $M \equiv(\lambda x . P) N \stackrel{\text { Top }}{\longrightarrow} * \equiv M^{\prime \prime}$.

Then $(\lambda x . P) N$ is of type $\mathbf{T}$, so also $P[N / x]$ is of type $\mathbf{T}$ and then $P[N / x] \stackrel{\mathbf{T}}{\longrightarrow}$ *

2. $M \stackrel{n}{\longrightarrow} M^{\prime}$.

2.1. If $M \Longrightarrow M^{\prime \prime}$ is internal:

2.1.1. $M \equiv M_{1} M_{2}, M^{\prime \prime} \equiv M_{1} M_{2}^{\prime}$ and $M_{2} \Longrightarrow M_{2}^{\prime}$

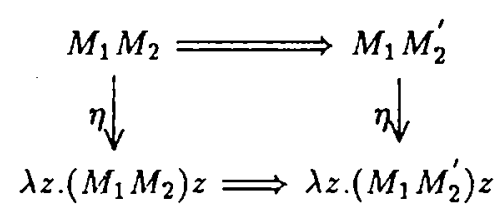

2.1.2. $M \equiv M_{1} M_{2}, M^{\prime \prime} \equiv M_{1}^{\prime} M_{2}$ and $M_{1} \Rightarrow M_{1}^{\prime}$

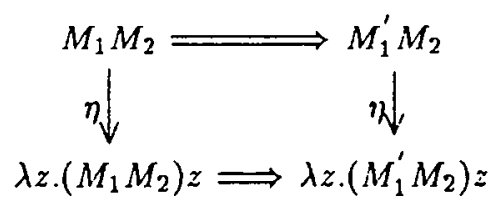

2.1.3. $M \equiv \pi_{i}(P), M^{\prime \prime} \equiv \pi_{i}\left(P^{\prime}\right)$ and $P \Longrightarrow P^{\prime}$

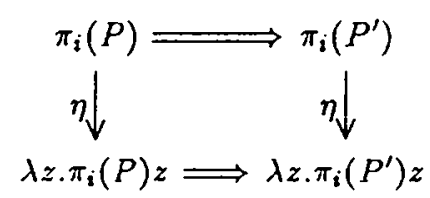


2.1.4. $M \equiv \operatorname{Case}\left(P, M_{1}, M_{2},\right), M^{\prime \prime} \equiv \operatorname{Case}\left(P^{\prime}, M_{1}, M_{2}\right)$ and $P \Longrightarrow P^{\prime}$

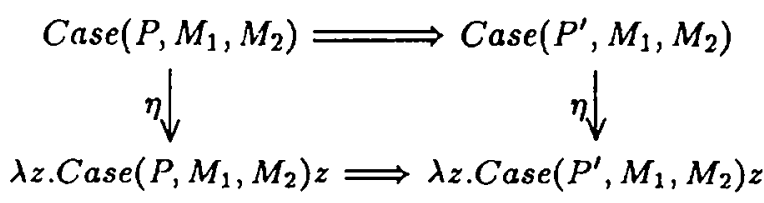

2.1.5. $M \equiv \operatorname{Case}\left(P, M_{1}, M_{2}\right), M^{\prime \prime} \equiv \operatorname{Case}\left(P, M_{1}^{\prime}, M_{2}\right)$ and $M_{1} \Longrightarrow M_{1}^{\prime}$

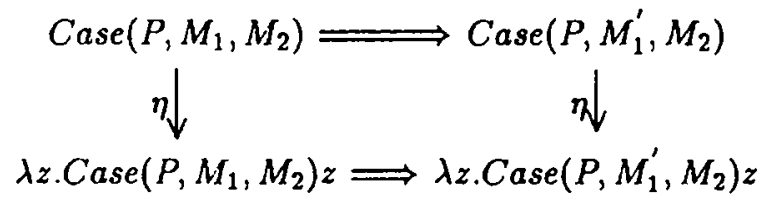

2.1.6. $M \equiv \operatorname{Case}\left(P, M_{1}, M_{2}\right), M^{\prime \prime} \equiv \operatorname{Case}\left(P, M_{1}, M_{2}^{\prime}\right)$ and $M_{2} \Longrightarrow M_{2}^{\prime}$

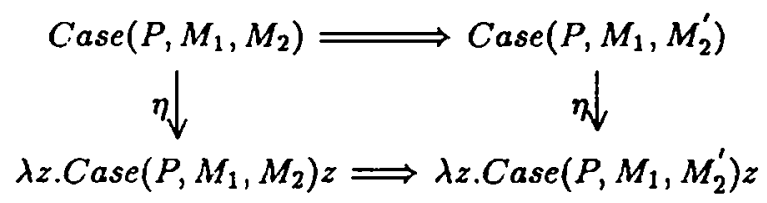

2.1.7. $M \equiv\left(\operatorname{rec} y \cdot M_{1}\right)^{i}, M^{\prime \prime} \equiv\left(\operatorname{rec} y \cdot M_{1}^{\prime}\right)^{i}$ and $M_{1} \Longrightarrow M_{1}^{\prime}$

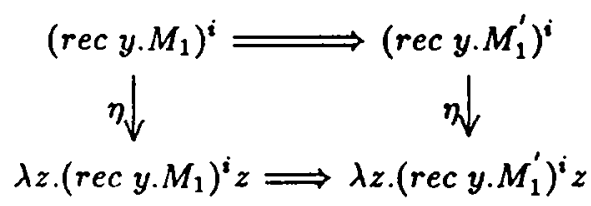

2.2. If $M \Longrightarrow M^{\prime \prime}$ is external, the cases to consider are:

2.2.1. $M \stackrel{\oplus}{\longrightarrow} M^{\prime \prime}$. This is the same as case 1.2.1

2.2.2. $M \stackrel{\pi_{i}}{\longrightarrow} M^{\prime \prime}$. Then $M \equiv \pi_{i}\left(\left(M_{1}, M_{2}\right\rangle\right)$ and there are two cases:

2.2.2.1. If $M_{i}$ is not a $\lambda$-abstraction, the diagram looks like:

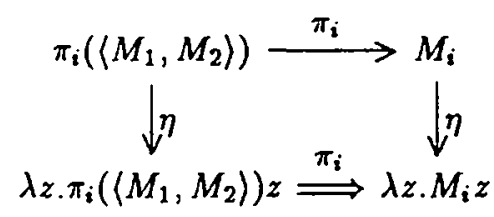

2.2.2.2. If $M_{i}$ is a $\lambda$-abstraction $\lambda y . M_{i}^{\prime}$, the diagram looks like:

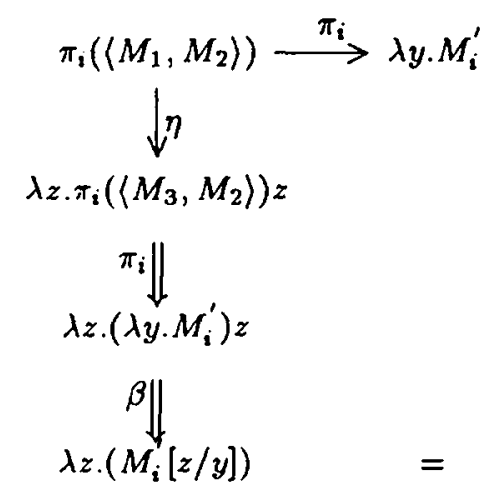


2.2.3. $M \stackrel{\bullet}{\longrightarrow} M^{\prime \prime}$. Then $M \equiv$ Case $\left(i n_{C_{1}+C_{2}}^{i}(P), M_{1}, M_{2}\right)$

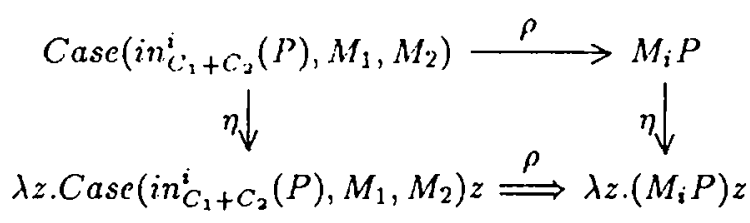

2.2.4. $M \stackrel{\text { rec }}{\longrightarrow} M^{\prime \prime}$. Then $M=\left(\text { rec } y \cdot M_{1}\right)^{i}$ and there are two possible cases:

2.2.4.1. If $M_{1}$ is not a $\lambda$-abstraction:

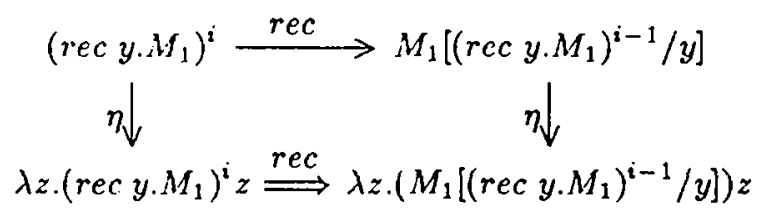

2.2.4.2. If $M_{1} \equiv \lambda w \cdot M_{1}^{\prime}$ :

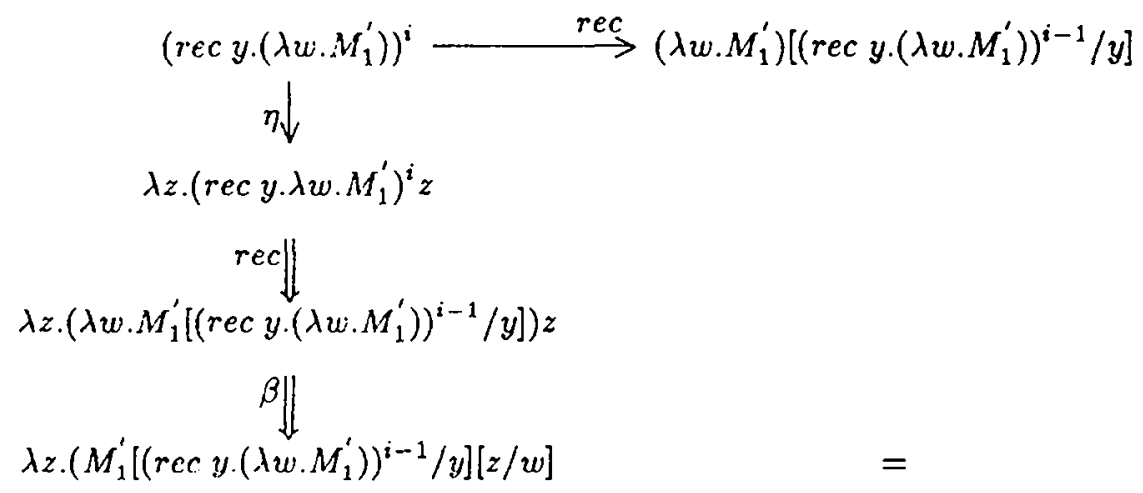

3. $M \stackrel{\text { Top }}{\longrightarrow} *$. Since $M$ is of type $\mathrm{T}$ and $M \Longrightarrow M^{\prime \prime}$, also $M^{\prime \prime}$ is of type $\mathrm{T}$ by proposition 3.7 . Then $M^{\prime \prime} \stackrel{\text { Top }}{\longrightarrow} *$.

4. $M \stackrel{\rightarrow}{\rightarrow} M^{\prime}$.

4.1. If $M \Longrightarrow M^{\prime \prime}$ is internal:

4.1.1. $M \equiv \pi_{1}\left(\left\langle M_{1}, M_{2}\right\rangle\right) \Longrightarrow \pi_{1}\left(\left\langle M_{1}^{\prime}, M_{2}\right\rangle\right) \equiv M^{\prime}$, where $M_{1} \Longrightarrow M_{1}^{\prime}$

$$
\begin{gathered}
\pi_{1}\left(\left\langle M_{1}, M_{2}\right\rangle\right) \Longrightarrow \pi_{1}\left(\left\langle M_{1}^{\prime}, M_{2}\right\rangle\right) \\
\pi_{1} \downarrow \\
M_{1} \Longrightarrow \pi_{1} \downarrow \\
M_{1}^{\prime}
\end{gathered}
$$

Idem for $M \equiv \pi_{2}\left(\left\langle M_{1}, M_{2}\right\rangle\right) \Longrightarrow \pi_{2}\left(\left\langle M_{1}, M_{2}^{\prime}\right\rangle\right) \equiv M^{\prime}$, where $M_{2} \Longrightarrow M_{2}^{\prime}$.

4.1.2. $M \equiv \pi_{1}\left(\left(M_{1}, M_{2}\right\rangle\right) \Longrightarrow \pi_{1}\left(\left\langle M_{1}, M_{2}^{\prime}\right\rangle\right) \equiv M^{\prime}$, where $M_{2} \Longrightarrow M_{2}^{\prime}$.

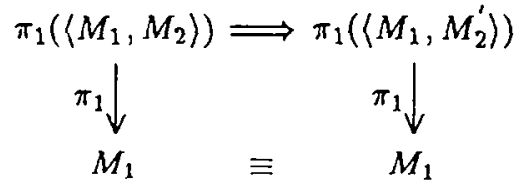

Idem for $M \equiv \pi_{2}\left(\left\langle M_{1}, M_{2}\right\rangle\right) \Longrightarrow \pi_{2}\left(\left\langle M_{1}^{\prime}, M_{2}\right\rangle\right) \equiv M^{\prime}$, where $M_{1} \Longrightarrow M_{1}^{\prime}$.

4.2. If $M \Longrightarrow M^{\prime \prime}$ is external: 
4.2.1. $M \stackrel{n}{\longrightarrow} M^{\prime \prime}$. This is the same as case 2.2.2.

4.2.2. $M \stackrel{\text { Top }}{\longrightarrow} M^{\prime \prime}$. This is considered in case 3 .

4.2.3. $M \stackrel{8}{\longrightarrow} M^{\prime \prime}$. Then $M \equiv \pi_{i}\left(\left\langle M_{1}, M_{2}\right\rangle\right)$.

4.2.3.1. If $M_{i}$ is not a pair:

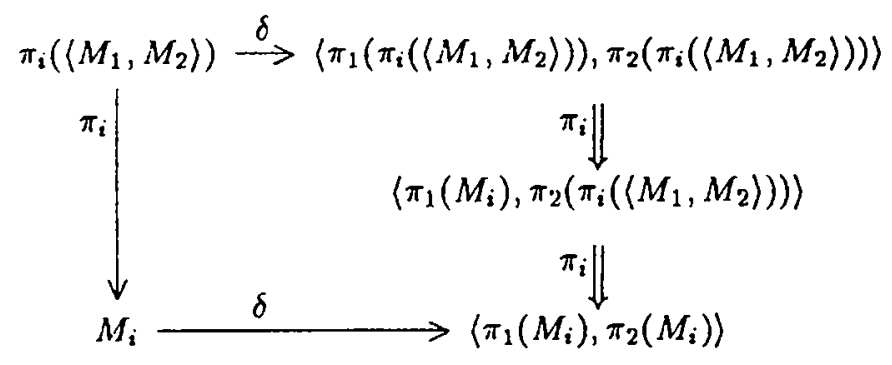

4.2.3.2. If $M_{i}$ is a pair $\left\langle P_{1}, P_{2}\right\rangle$ :

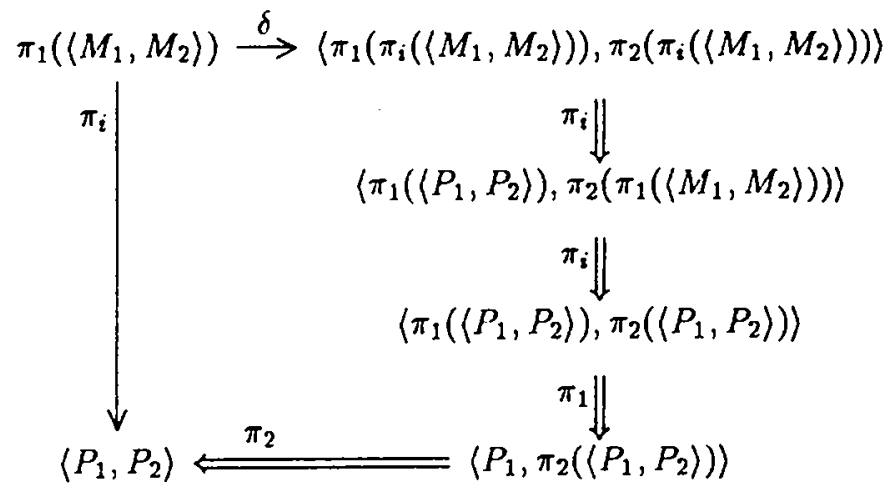

5. $M \stackrel{6}{\longrightarrow} M^{\prime}$.

5.1. If $M \Rightarrow M^{\prime \prime}$ is internal:

5.1.1. $M \equiv M_{1} M_{2} \Longrightarrow M_{1}^{\prime} M_{2} \equiv M^{\prime \prime}$, where $M_{1} \Longrightarrow M_{1}^{\prime}$,

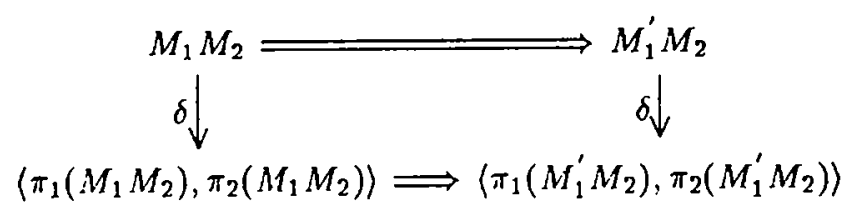

5.1.2. $M \equiv M_{1} M_{2} \Longrightarrow M_{1} M_{2}^{\prime} \equiv M^{\prime \prime}$, where $M_{2} \Longrightarrow M_{2}^{\prime}$;

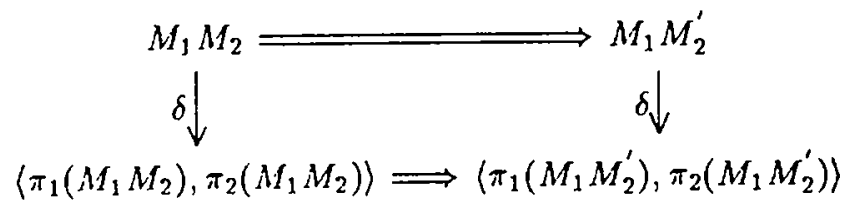

5.1.3. $M \equiv \pi_{i}\left(M_{1}\right) \Longrightarrow \pi_{i}\left(M_{1}^{\prime}\right) \equiv M^{\prime \prime}$, where $M_{1} \Rightarrow M_{1}^{\prime}$,

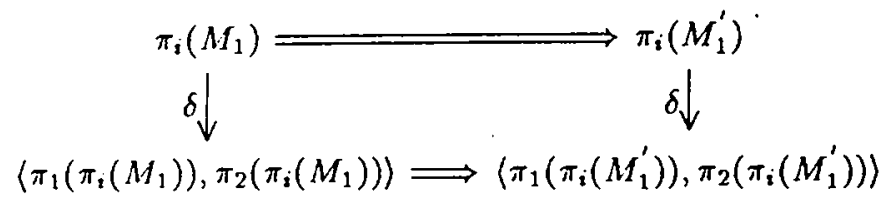


5.1.4. $M \equiv \operatorname{Case}\left(P, M_{1}, M_{2}\right) \Longrightarrow \operatorname{Case}\left(P^{\prime}, M, N\right) \equiv M^{\prime \prime}$, where $P \Longrightarrow P^{\prime}$.

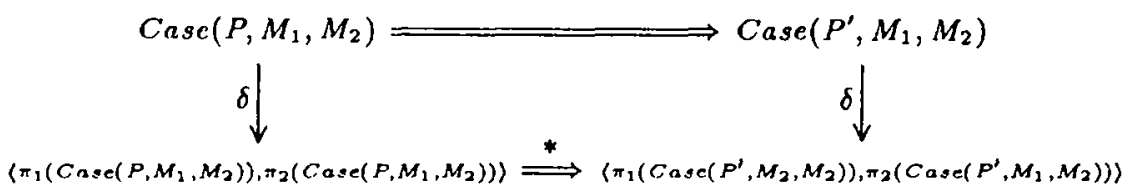

5.1.5. $M \equiv \operatorname{Casc}\left(P, M_{1}, M_{2}\right) \Longrightarrow \operatorname{Case}\left(P, M_{1}^{\prime}, M_{2}\right) \equiv M^{\prime \prime}$, where $M_{1} \Longrightarrow M_{1}^{\prime}$.

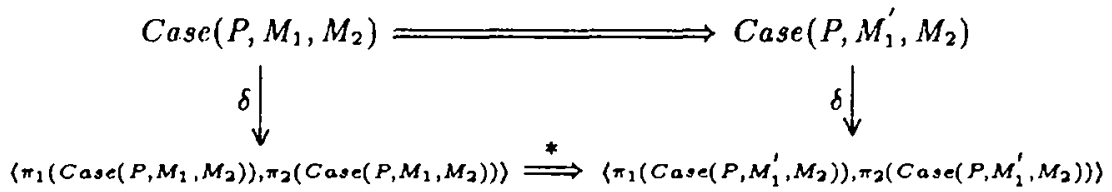

5.1.6. $M \equiv \operatorname{Case}\left(P, M_{1}, M_{2}\right) \Longrightarrow \operatorname{Case}\left(P, M_{1}, M_{2}\right) \equiv M^{\prime \prime}$, where $M_{2} \Longrightarrow M_{2}^{\prime}$.

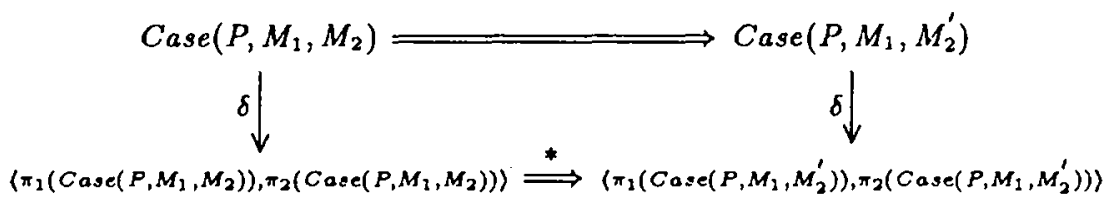

5.1.7. $(\text { rec } y: C . P)^{i} \Longrightarrow\left(\text { rec } y: C . P^{\prime}\right)^{i}$, where $P \Longrightarrow P^{\prime}$.

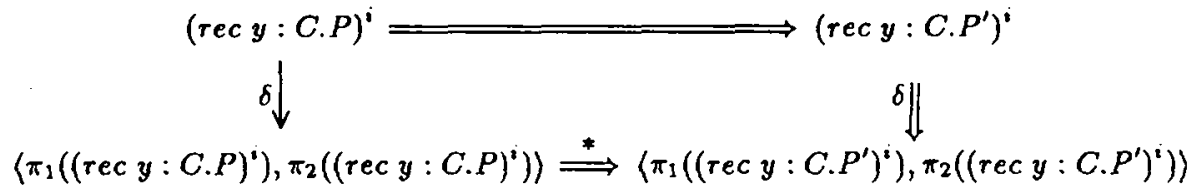

5.2. If $M \Longrightarrow M^{\prime \prime}$ is external:

5.2.1. $M \stackrel{B}{\longrightarrow} M^{\prime \prime}$. This is the same as case 1.2 .2

5.2.2. $M \stackrel{{ }^{*}}{\longrightarrow} M^{\prime \prime}$. This is the same as case 4.2.3

5.2.3. $M \equiv \operatorname{Case}\left(i_{C_{1}+C_{2}}^{i}(P), M_{1}, M_{2}\right) \stackrel{\circ}{\longrightarrow} M_{i} P \equiv M^{\prime \prime}$.

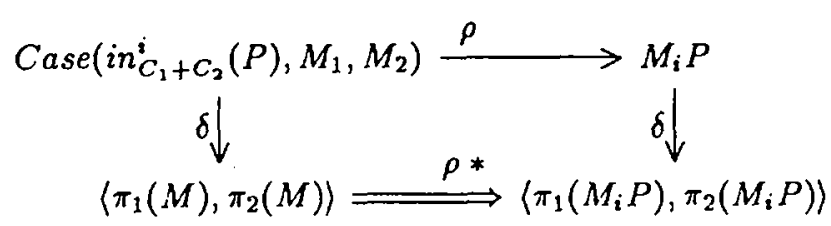

5.2.4. $M \equiv(\operatorname{rec} y: C . P)^{i} \rightarrow P\left[(\text { rec } y: C . P)^{i-1} / y\right] \equiv M^{\prime \prime}$.

5.2.4.1. If $P$ is not a pair:

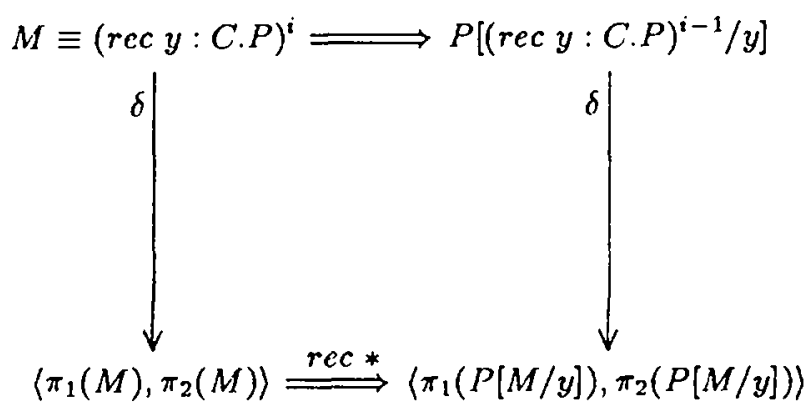


5.2.4.2. If $P$ is a pair $\left\langle P_{1}, P_{2}\right\rangle$ :

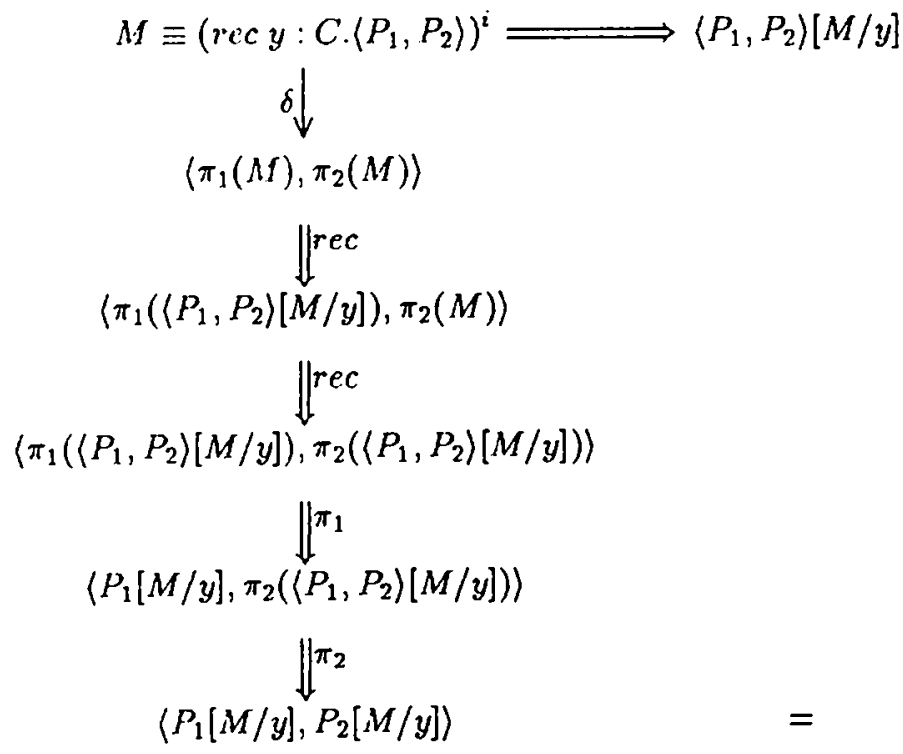

6. $M \stackrel{\circ}{\longrightarrow} M^{\prime}$.

6.1. If $M \Longrightarrow M^{\prime \prime}$ is internal:

6.1.1. $\operatorname{Case}\left(\operatorname{in}_{C}^{i}(P), M_{1}, M_{2}\right) \Longrightarrow \operatorname{Case}\left(i_{C}^{i}\left(P^{\prime}\right), M_{1}, M_{2}\right)$, where $P \Longrightarrow P^{\prime}$.

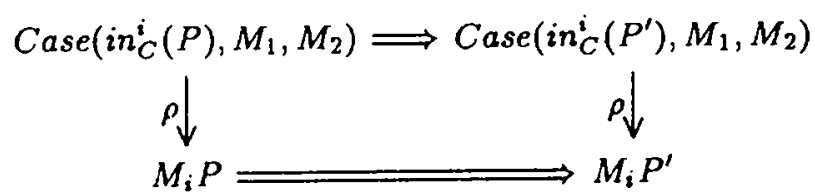

6.1.2. Case(in $\left.{ }_{C}^{1}(P), M_{1}, M_{2}\right) \Longrightarrow \operatorname{Case}\left(\operatorname{in}_{C}^{1}(P), M_{1}^{\prime}, M_{2}\right)$, where $M_{1} \Longrightarrow M_{1}^{\prime}$ but $M_{1} \stackrel{\neg n}{\longrightarrow} M_{1}^{\prime}$

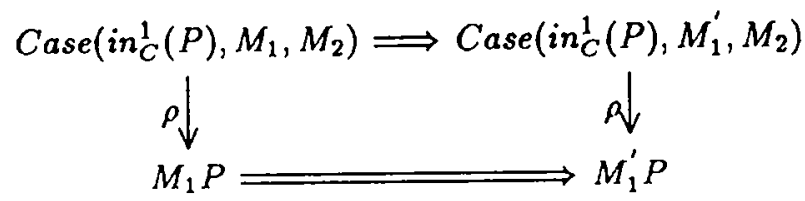

Idem for $\operatorname{Case}\left(\operatorname{in}_{C}^{2}(P), M_{1}, M_{2}\right) \Longrightarrow \operatorname{Case}\left(i n_{C}^{2}(P), M_{1}, M_{2}^{\prime}\right)$, where $M_{2} \stackrel{\rightarrow n}{\longrightarrow} M_{2}^{\prime}$.

6.1.3. Case $\left(\operatorname{in}_{C}^{1}(P), M_{1}, M_{2}\right) \Longrightarrow$ Case $\left(\operatorname{in}_{C}^{1}(P), M_{1}^{\prime}, M_{2}\right)$, where $M_{1} \longrightarrow \lambda x \cdot M_{1} x$

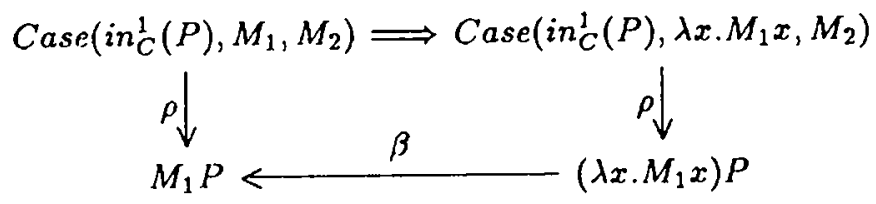

Idem for $\operatorname{Case}\left(\operatorname{in}_{C}^{2}(P), M_{1}, M_{2}\right) \Longrightarrow \operatorname{Case}\left(i_{C}^{2}(P), M_{1}, M_{2}^{\prime}\right)$, where $M_{2} \longrightarrow \lambda x . M_{2} x$

6.2. If $M \Longrightarrow M^{\prime \prime}$ is external:

6.2.1. $M \stackrel{n}{\longrightarrow} M^{\prime \prime}$. This is the same as case 2.2.3.

6.2.2. $M \stackrel{\text { Top }}{\longrightarrow} M^{\prime \prime}$. This is considered in case 3 .

6.2.3. $M \stackrel{8}{\longrightarrow} M^{\prime \prime}$. This is the same as case 5.2.3.

7. $M \stackrel{\text { rec }}{\longrightarrow} M^{\prime}$. 
7.1. If $M \Longrightarrow M^{\prime \prime}$ is internal:

7.1.1. $(\text { rec } y: C . P)^{i} \Longrightarrow\left(\text { rec } y: C . P^{\prime}\right)^{i}$, where $P \Longrightarrow P^{\prime}$. We have $P\left[(\text { rec } y: C . P)^{i-1 / y}\right]$ $\Longrightarrow P\left[\left(\text { rec } y: C . P^{\prime}\right)^{i-1} / y\right]$ by lemma 4.8 and by lemma $4.5 P\left[\left(\text { rec } y: C . P^{\prime}\right)^{i-1} / y\right]$ $\Longrightarrow^{*} P^{\prime}\left[\left(\operatorname{rec} y: C \cdot P^{\prime}\right)^{i-1} / y\right]$ or $P^{\prime}\left[\left(\operatorname{rec} y: C \cdot P^{\prime}\right)^{i-1} / y\right] \Longrightarrow^{*} P\left[\left(\operatorname{rec} y: C . P^{\prime}\right)^{i-1} / y\right]$. In the first case:

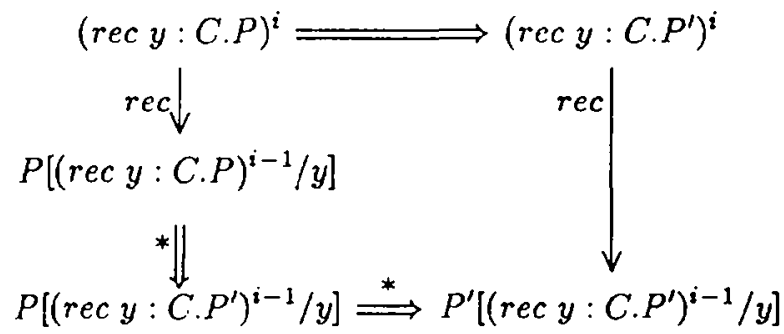

In the second case:

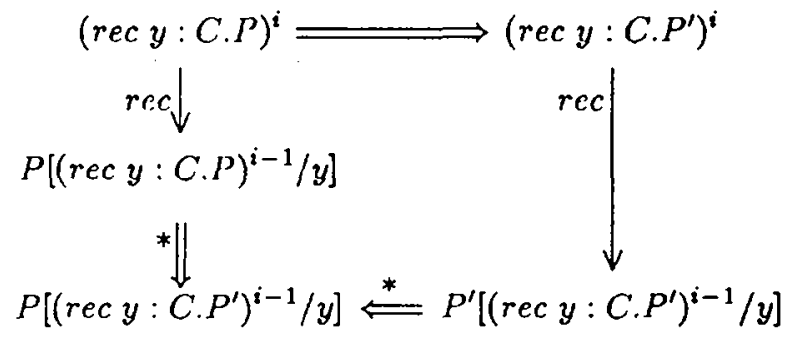

7.2. If $M \Longrightarrow M^{\prime \prime}$ is external:

7.2.1. $M \stackrel{\rightarrow}{\longrightarrow} M^{\prime \prime}$. This is the same as case 2.2.4.

7.2.2. $M \stackrel{\text { Top }}{\longrightarrow} M^{\prime \prime}$. This is considered in case 3 .

7.2.3. $M \stackrel{\circ}{\longrightarrow} M^{\prime \prime}$. This is the same as case 5.2.4.

\subsection{From Solved Critical Pairs to Full Weak Confluence}

It is to be noted that the solvability of critical pairs we just proved as Proposition 4.9 does not allow us to deduce the weak confluence of the calculus via the famous Knuth-Bendix Critical Pairs Lemma. That Lemma holds only for algebraic rewrite systems, and not for the $\lambda$-calculus, that has the higher order rewrite rule $\beta$. We need to prove local confluence explicitly, and to do so the following remark is useful.

Remark 4.10 (Expansion rules) In case the two reductions $M^{\prime} \longleftarrow M \Longrightarrow M^{\prime \prime}$ do not involve $\eta$ (resp. $\delta$ ) rules applied at the root positions of $M$, it is possible to close the diagram without using $\eta$ (resp. $\delta$ ) rules at the root, except in the three cases shown below: external $\pi$ 's and internal $\eta$, external $\beta$ and internal $\delta$. Notice that $M$ is not a $\lambda$-abstraction in the first diagram, $N$ is not a $\lambda$-abstraction in the second and $M[N / x]$ is not a pair in the third one.

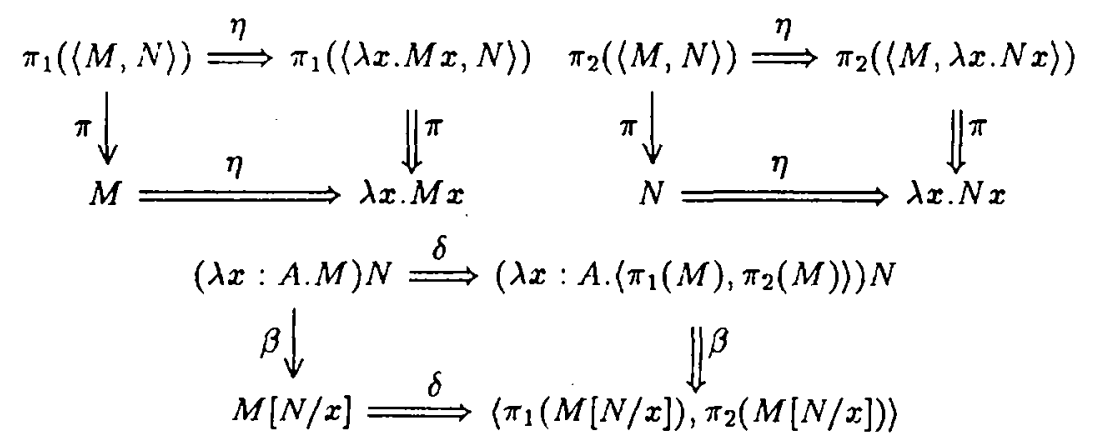


With this additional knowledge, we can prove that $\Longrightarrow$ is actually weakly confluent.

Theorem 4.11 (Weak Confluence) If $M^{\prime} \Longleftarrow M \Rightarrow M^{\prime \prime}$ then there exist a term $M^{\prime \prime \prime}$ such that $M^{\prime} \Longrightarrow^{*} M^{\prime \prime \prime}{ }^{*} M^{\prime \prime}$ (i.e. the reduction relation $\Longrightarrow$ is weakly confluent). Furthermore, if the reductions in $M^{\prime} \Longleftarrow M \Longrightarrow M^{\prime \prime}$ do not contain $\eta$ (resp. $\delta$ ) rules applied at the root of $M$, it is possible also to close the diagram without applying $\eta$ (resp. $\delta$ ) rules at the root, except in the cases shown in the previous Remark 4.10.

Proof. We will prove that there exists a term $M^{\prime \prime \prime}$ such that $M^{\prime} \Longrightarrow^{*} M^{\prime \prime \prime}{ }^{*} \Longleftarrow M^{\prime \prime}$, by induction on the derivation of $M \Longrightarrow M^{\prime}$. First of all, we remark that if one of the two one-step reductions $M \Longrightarrow M^{\prime}$ and $M \Longrightarrow M^{\prime \prime}$ is actually an external reduction $M \stackrel{M}{\longrightarrow}$ 'and $M \stackrel{M}{\longrightarrow}$ ", then the result comes directly from Proposition 4.9. So we will need to consider in the following only the cases where both reductions are internal reductions.

We proceed now by cases on the last rule used to derive $M \Longrightarrow M^{\prime}$.

- $M \equiv\left(M_{1} M_{2}\right) \Longrightarrow\left(M_{1}^{\prime} M_{2}\right) \equiv M^{\prime}$ comes from $M_{1} \Longrightarrow M_{1}^{\prime}$. In this case, the $\eta$ rule cannot be applied at the root position of $M_{1}$ because $M_{1}$ is evaluated. Then we have two cases:

- the reduction $M \equiv\left(M_{1} M_{2}\right) \Longrightarrow\left(M_{1}^{\prime \prime} M_{2}\right) \equiv M^{\prime \prime}$ comes from a reduction $M_{1} \Longrightarrow M_{1}^{\prime \prime}$. Now we have to consider two cases:

* $M_{1}^{\prime} \Longleftarrow M_{1} \Longrightarrow M_{1}^{\prime \prime}$ is not one of the exceptional cases for $\eta$ of the Remark 4.10: then we know that there are no $\eta$ at the root position in $M_{1}^{\prime} \Longrightarrow^{*} M_{1}^{\prime \prime \prime}{ }^{*} \Longleftarrow M_{1}^{\prime \prime}$. By induction hypothesis we get a term $M_{1}^{\prime \prime \prime}$ that can be used to close the diagram $M_{1}^{\prime} \Longleftarrow M_{1} \Longrightarrow M_{1}^{\prime \prime}$ via $M_{1}^{\prime} \Longrightarrow^{*} M_{1}^{\prime \prime \prime}{ }^{*} \Longleftarrow M_{1}^{\prime \prime}$, and we can close our original diagram with

$$
M^{\prime} \equiv\left(M_{1}^{\prime} M_{2}\right) \Longrightarrow^{*}\left(M_{1}^{\prime \prime \prime} M_{2}\right)^{*} \Longleftarrow\left(M_{1}^{\prime \prime} M_{2}\right) \equiv M^{\prime \prime}
$$

* $M_{1}^{\prime} \Longleftarrow M_{1} \Longrightarrow M_{1}^{\prime \prime}$ is one of the exceptional cases for $\eta$, hence $M_{1}$ is $\pi_{1}(\langle P, Q\rangle)$ for some terms $P$ and $Q$. We can still close the original diagram as follows:

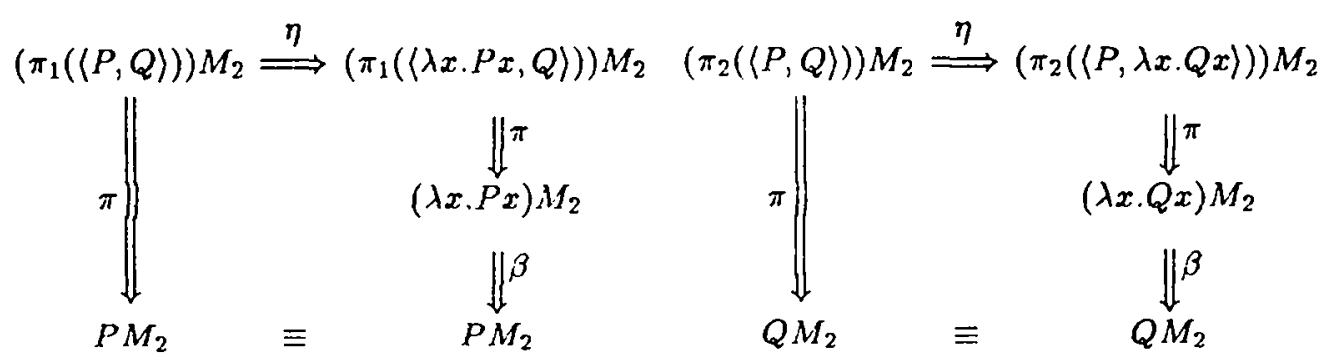

- the reduction $M \equiv\left(M_{1} M_{2}\right) \Longrightarrow\left(M_{1} M_{2}^{\prime \prime}\right) \equiv M^{\prime \prime}$ comes from a reduction $M_{2} \Rightarrow M_{2}^{\prime \prime}$. We can close the diagram using the same original reductions,

$$
M^{\prime} \equiv\left(M_{1}^{\prime} M_{2}\right) \Longrightarrow\left(M_{1}^{\prime} M_{2}^{\prime \prime}\right) \Longleftarrow\left(M_{1} M_{2}^{\prime \prime}\right) \equiv M^{\prime \prime}
$$

because we know that $\eta$ is not applied to $M_{1}$ to get to $M_{1}^{\prime}$.

- $M \equiv\left(M_{1} M_{2}\right) \Longrightarrow\left(M_{1} M_{2}^{\prime}\right) \equiv M^{\prime}$ comes from $M_{2} \Longrightarrow M_{2}^{\prime}$. Then we have two cases:

- the reduction $M \equiv\left(M_{1} M_{2}\right) \Longrightarrow\left(M_{1} M_{2}^{\prime \prime}\right) \equiv M^{\prime \prime}$ comes from a reduction $M_{2} \Longrightarrow M_{2}^{\prime \prime}$. By induction hypothesis we get a term $M_{2}^{\prime \prime \prime}$ that can be used to close $M_{2}^{\prime} \Longleftarrow M_{2} \Longrightarrow M_{2}^{\prime \prime}$ via $M_{2}^{\prime} \Longrightarrow^{*} M_{2}^{\prime \prime \prime}{ }^{*} \Longleftarrow M_{2}^{\prime \prime}$. Now $M^{\prime} \equiv\left(M_{1} M_{2}^{\prime}\right) \Longrightarrow^{*}\left(M_{1} M_{2}^{\prime \prime \prime}\right)^{*} \Longleftarrow\left(M_{1} M_{2}^{\prime \prime}\right) \equiv M^{\prime \prime}$ can be used to close our original diagram.

- the reduction $M \equiv\left(M_{1} M_{2}\right) \Longrightarrow\left(M_{1}^{\prime \prime} M_{2}\right) \equiv M^{\prime \prime}$ comes from a reduction $M_{1} \Longrightarrow M_{1}^{\prime \prime}$. In this case, we know that $\eta$ cannot be applied at the top to $M_{1}$ to get to $M_{1}^{\prime \prime}$ because $M_{1}$ is evaluated. So, we can close the diagram using the same original reductions, $M^{\prime} \equiv$ $\left(M_{1} M_{2}^{\prime}\right) \Longrightarrow\left(M_{1}^{\prime \prime} M_{2}^{\prime}\right) \Longleftarrow\left(M_{1}^{\prime \prime} M_{2}\right) \equiv M^{\prime \prime}$. 
- $M \equiv\left(\pi_{i}\left(M_{1}\right)\right) \Longrightarrow\left(\pi_{i}\left(M_{1}^{\prime}\right)\right) \equiv M^{\prime}$ comes from $M_{1} \Longrightarrow M_{1}^{\prime}$ and $M \equiv\left(\pi_{i}\left(M_{1}\right)\right) \Longrightarrow\left(\pi_{i}\left(M_{1}^{\prime \prime}\right)\right) \equiv$ $M^{\prime \prime}$ comes from $M_{1} \Longrightarrow M_{1}^{\prime \prime}$. Then neither $M_{1} \Longrightarrow M_{1}^{\prime}$ nor $M_{1} \Longrightarrow M_{1}^{\prime \prime}$ can use $\delta$ rules at the root of $M$ because it is projected.

Now we have two cases:

- $M_{1} \Longrightarrow M_{1}^{\prime}$ and $M_{1} \Rightarrow M_{1}^{\prime \prime}$ are not the exceptional cases for $\delta$ of Remark 4.10. By induction hypothesis there is an $M_{1}^{\prime \prime \prime}$ s.t. $M_{1}^{\prime} \Longrightarrow^{*} M_{1}^{\prime \prime \prime} \Longleftarrow M_{1}^{\prime \prime}$ without $\delta$ rules at the root, and we can close our diagram by $\pi_{i}\left(M_{1}^{\prime}\right) \Longrightarrow{ }^{*} \pi_{i}\left(M_{1}^{\prime \prime \prime}\right){ }^{*} \Longleftarrow \pi_{i}\left(M_{1}^{\prime \prime}\right)$.

- $M_{1} \Longrightarrow M_{1}^{\prime}$ and $M_{1} \Longrightarrow M_{1}^{\prime \prime}$ is the exceptional case for $\delta$, so $M_{1} \equiv(\lambda x . P) Q$ for some terms $P$ and $Q$. We can still close our original diagram as follows:

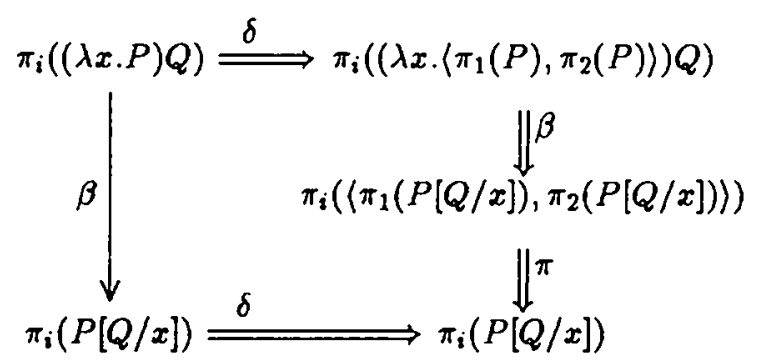

- $M \equiv \lambda x \cdot M_{1} \Longrightarrow \lambda x \cdot M_{1}^{\prime} \equiv M^{\prime}$ comes from $M_{1} \Longrightarrow M_{1}^{\prime}$ and $M \equiv \lambda x \cdot M_{1} \Longrightarrow \lambda x \cdot M_{1}^{\prime \prime} \equiv M^{\prime \prime}$ comes from $M_{1} \Longrightarrow M_{1}^{\prime \prime}$. By induction hypothesis there is an $M_{1}^{\prime \prime \prime}$ s.t. $M_{1}^{\prime} \Longrightarrow^{*} M_{1}^{\prime \prime \prime}{ }^{*} \Longleftarrow M_{1}^{\prime \prime}$ and we can close our diagram by $\lambda x . M_{1}^{\prime} \Longrightarrow^{*} \lambda x \cdot M_{1}^{\prime \prime \prime}{ }^{*} \Longleftarrow \lambda x \cdot M_{1}^{\prime \prime}$.

- $M \equiv\left\langle M_{1}, M_{2}\right\rangle \Longrightarrow\left\langle M_{1}^{\prime}, M_{2}\right\rangle \equiv M^{\prime}$ comes from $M_{1} \Longrightarrow M_{1}^{\prime}$. Now we have to consider two cases:

- the reduction $M \equiv\left\langle M_{1}, M_{2}\right\rangle \Longrightarrow\left\langle M_{1}, M_{2}^{\prime \prime}\right\rangle \equiv M^{\prime \prime}$ comes from a reduction $M_{2} \Longrightarrow M_{2}^{\prime \prime}$ By induction hypothesis there is a term $M_{1}^{\prime \prime \prime}$ s.t. we can close the diagram $M_{1}^{\prime} \Longleftarrow M_{1} \Longrightarrow M_{1}^{\prime \prime}$ via $M_{1}^{\prime} \Longrightarrow^{*} M_{1}^{\prime \prime \prime}{ }^{*} \Longleftarrow M_{1}^{\prime \prime}$, and we can close our original diagram with

$$
M^{\prime} \equiv\left\langle M_{1}^{\prime}, M_{2}\right\rangle \Longrightarrow^{*}\left\langle M_{1}^{\prime \prime \prime}, M_{2}\right\rangle^{*} \Longleftarrow\left\langle M_{1}^{\prime \prime}, M_{2}\right\rangle \equiv M^{\prime \prime}
$$

- the reduction $M \equiv\left\langle M_{1}, M_{2}\right\rangle \Longrightarrow\left\langle M_{1}, M_{2}^{\prime \prime}\right\rangle \equiv M^{\prime \prime}$ comes from a reduction $M_{2} \Longrightarrow M_{2}^{\prime \prime}$. We can close the diagram using the same original reductions,

$$
M^{\prime} \equiv\left\langle M_{1},{ }^{\prime} M_{2}\right\rangle \Longrightarrow\left\langle M_{1}^{\prime}, M_{2}^{\prime \prime}\right\rangle \Longleftarrow\left\langle M_{1}, M_{2}^{\prime \prime}\right\rangle \equiv M^{\prime \prime}
$$

- $M \equiv i n_{C}^{i}\left(M_{1}\right) \Longrightarrow i n_{C}^{i}\left(M_{1}^{\prime}\right) \equiv M^{\prime}$ comes from $M_{1} \Longrightarrow M_{1}^{\prime}$ and $M \equiv i n_{C}^{i}\left(M_{1}\right) \Longrightarrow i n_{C}^{i}\left(M_{1}^{\prime \prime}\right) \equiv$ $M^{\prime \prime}$ comes from $M_{1} \Longrightarrow M_{1}^{\prime \prime}$. By induction hypothesis there is an $M_{1}^{\prime \prime \prime}$ s.t. $M_{1}^{\prime} \Longrightarrow{ }^{*} M_{1}^{\prime \prime \prime}{ }^{*} \Longleftarrow M_{1}^{\prime \prime}$ and we can close our diagram by $i n_{C}^{i}\left(M_{1}^{\prime}\right) \Longrightarrow \Longrightarrow^{*} i n_{C}^{i}\left(M_{1}^{\prime \prime \prime}\right)^{*} \Longleftarrow i n_{C}^{i}\left(M_{1}^{\prime \prime}\right)$.

- $M \equiv \operatorname{rec} x: A \cdot M_{1} \Longrightarrow \operatorname{rec} x: A . M_{1}^{\prime} \equiv M^{\prime}$ comes from $M_{1} \Longrightarrow M_{1}^{\prime}$ and $M \equiv \operatorname{rec} x$ : $A . M_{1} \Longrightarrow \operatorname{rec} x: A . M_{1}^{\prime \prime} \equiv M^{\prime \prime}$ comes from $M_{1} \Longrightarrow M_{1}^{\prime \prime}$. Then we can find by induction hypothesis an $M_{1}^{\prime \prime \prime}$ s.t. $M_{1}^{\prime} \Longrightarrow^{*} M_{1}^{\prime \prime \prime} \Longleftarrow M_{1}^{\prime \prime}$ and we can close our diagram by rec $x$ : $A \cdot M_{1}^{\prime} \Longrightarrow^{*} \operatorname{rec} x: A \cdot M_{1}^{\prime \prime \prime} \rightleftharpoons \operatorname{rec} x: A \cdot M_{1}^{\prime \prime}$.

- We are left to consider the case of $M \equiv \operatorname{Case}\left(P, M_{1}, M_{2}\right)$.

- To avoid a mechanical repetition of similar proofs, notice that if the internal reduction to $M^{\prime}$ and $M^{\prime \prime}$ are performed on different subterms, then we can close the diagram by commuting the two reductions. We show just one case.

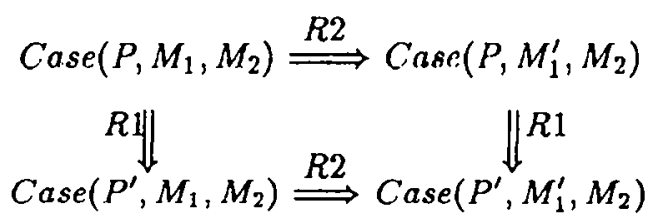


- If the internal reduction to $M^{\prime}$ and $M^{\prime \prime}$ are performed on the same subterm $Q$, say $Q^{\prime} \stackrel{R 1}{=} Q \stackrel{R 2}{=} Q^{\prime \prime}$, then there is a $Q^{\prime \prime \prime}$, by induction hypothesis, s.t. $Q^{\prime} \Longrightarrow * Q^{\prime \prime \prime}{ }^{*} Q^{\prime \prime}$, and we can close the diagram by extending these last reductions to the Case expression. Again, we detail just one case.

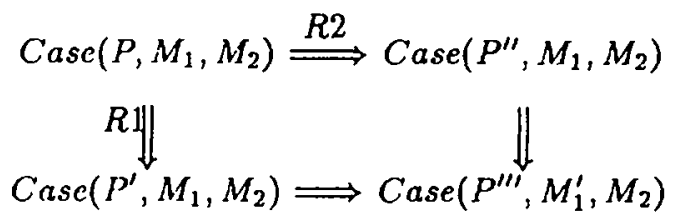

\section{Strong Normalization}

We provide in this section the proof of strong normalization for our calculus. The key idea is to reduce strong normalization of the system with expansion rules to that of the system without expansion rules and for this, we show how the calculus without expansions can be used to simulate the calculus with expansions. We will use a fundamental property relating strong normalization of two systems:

Proposition 5.1 Let $\mathcal{R}_{1}$ and $\mathcal{R}_{2}$ be two reduction systems and $T$ a translation from terms in $\mathcal{R}_{1}$ to terms in $\mathcal{R}_{2}$. If for every reduction $M_{1} \stackrel{\mathcal{R}_{1}}{\Longrightarrow} M_{2}$ there is a non empty reduction sequence $P_{1} \stackrel{\mathcal{R}_{2}}{\Longrightarrow}+P_{2}$ such that $\mathcal{T}\left(M_{i}\right)=P_{i}$, for $i=1,2$, then the strong normalization of $\mathcal{R}_{2}$ implies that of $\mathcal{R}_{1}$.

Proof. Suppose $\mathcal{R}_{2}$ is strongly normalizing and $\mathcal{R}_{1}$ is not. Then there is an infinite reduction sequence $M_{1} \stackrel{\mathcal{R}_{2}}{\Longrightarrow} M_{2} \stackrel{\mathcal{R}_{2}}{\Longrightarrow} \ldots$ and from this reduction we can construct an infinite reduction sequence $\mathcal{T}\left(M_{1}\right) \stackrel{\mathbb{R}_{2}}{\Longrightarrow}+\mathcal{T}\left(M_{2}\right) \stackrel{\mathbb{R}_{2}}{\Longrightarrow}+\ldots$ which leads to a contradiction.

The goal is now to find a translation of terms mapping our calculus into itself such that for every possible reduction in the original system from a term $M$ to another term $N$, there is a reduction sequence from the translation of $M$ to the translation of $N$, that is non empty and does not contain any expansion. Then the previous proposition allows us to derive the strong normalization property for the full system from that of the system without expansion rules, which can be proved using standard techniques.

\subsection{Simulating Expansions without Expansions}

The first naive idea that comes to the mind is to choose a translation such that expansion rules are completely impossible on a translated term. This essentially amounts to associate to a term $M$ its $\eta-\delta$ normal form, so that translating a term corresponds then to executing all the possible expansions.

Unfortunately, this simple solution is not a good one: if $M$ reduces to $N$ via an expansion, then the translation of $M$ and that of $N$ are the same term, so to such a reduction step in the full system corresponds an empty reduction sequence in the translation, and this does not allow us to apply proposition 5.1 .

This leads us to consider a more sophisticated translation that maps a term $M$ to a term $M^{\circ}$ where expansions are not fully executed as above, but just marked in such a way that they can be executed during the simulation process, if necessary, by a rule that is not an expansion.

Let us see how to do this on a simple example: take a variable $z$ of type $A_{1} \times A_{2}$, where the $A_{i}$ 's are atomic types different from $\mathbf{T}$. By performing a $\delta$ expansion we obtain its normal form w.r.t. expansion rules: $\left\langle\pi_{1}(z), \pi_{2}(z)\right)$. Instead of executing this reduction, we just mark it in the translation by applying to $z$ an appropriate expansor term $\lambda x: A_{1} \times A_{2} \cdot\left\langle\pi_{1}(x), \pi_{2}(x)\right\rangle$. As for $\left\langle\pi_{1}(z), \pi_{2}(z)\right\rangle$, it is in normal form w.r.t. expansions, so the translation does not modify it in any way. Now, we have the reduction sequence

$$
z^{0} \equiv\left(\lambda x: A_{1} \times A_{2} \cdot\left\langle\pi_{1}(x), \pi_{2}(x)\right\rangle\right) z \rightarrow_{\beta}\left\langle\pi_{1}(z), \pi_{2}(z)\right\rangle
$$


where the translation of $z$ reduces to the translation of $\left\langle\pi_{1}(z), \pi_{2}(z)\right\rangle$. and the $\delta$ expansion from $z$ to $\left\langle\pi_{1}(z), \pi_{2}(z)\right\rangle$ is simulated in the translation by a $\beta$-rule. Clearly, in a generic term $M$ there are many positions where an expansion can be performed, so the translation will have to take into account the structure of $M$ and insert the appropriate expansors at all these positions ${ }^{5}$.

Anyway, expansors must be carefully defined to correctly represent not only the expansion step arising from a redex already present in $M$, but also all the expansion sequences that such step can create: if in the previous example the type $A_{1}$ is taken to be an arrow type and the type $A_{2}$ a product type, then the term $\pi_{1}(z)$ can be further $\eta$-expanded and the term $\pi_{2}(z)$ can be expanded by a $\delta$-rule, and the expansor $\lambda x: A_{1} \times A_{2} \cdot\left\langle\pi_{1}(x), \pi_{2}(x)\right\rangle$ cannot simulate these further possible reductions. This can only be done by storing in the expansor terms all the information on possible future expansions, that is fully contained in the type of the term we are marking.

Definition 5.2 (Translation) To every type $C$ we associate a term, called the expansor of type $C$ and denoted $\Delta_{C}$, defined by induction as follows:

$$
\begin{array}{ll}
\Delta_{A \rightarrow B}= & \lambda x: A \rightarrow B \cdot \lambda z: A \cdot \Delta_{B}\left(x\left(\Delta_{A} z\right)\right) \\
\Delta_{A \times B}= & \lambda x: A \times B \cdot\left(\Delta_{A}\left(\pi_{1}(x)\right), \Delta_{B}\left(\pi_{2}(x)\right)\right) \\
\Delta_{A} & \text { is empty, in any other case }
\end{array}
$$

We then define a translation $M^{\circ}$ for a term $M: A$ as follows:

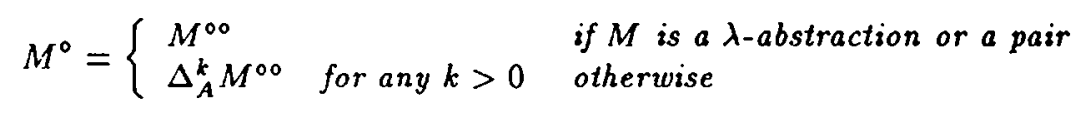

where $\Delta_{A}^{k} M$ denotes the term $\underbrace{\left(\Delta_{A} \ldots\left(\Delta_{A}\right.\right.}_{k \text { times }} M) \ldots)$ and $M^{\circ 0}$ is defined by induction as:

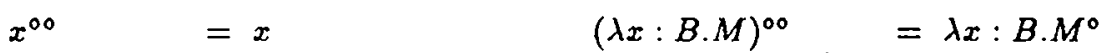

$$
\begin{aligned}
& *^{\circ 0} \quad=* \quad(\operatorname{rec} y: A \cdot M)^{i^{00}} \quad=\left(\operatorname{rec} y: A \cdot M^{\circ}\right)^{i} \\
& \langle M, N\rangle^{\circ 0}=\left\langle M^{\circ}, N^{\circ}\right\rangle \quad \operatorname{Case}(R, M, N)^{\circ 0}=\operatorname{Case}\left(R^{\circ}, M^{\circ}, N^{\circ}\right) \\
& (M N)^{\circ 0}=\left(M^{\circ 0} N^{0}\right) \quad \pi_{i}(M)^{\circ 0}=\pi_{i}\left(M^{\circ 0}\right) \\
& i n_{C}^{i}(M)^{00}=i n_{C}^{i}\left(M^{0}\right)
\end{aligned}
$$

This corresponds exactly to the marking procedure described before, but for a little detail: in the translation we allow any number of markers to be used (the integer $k$ can be any positive number), and not just one as seemed to suffice for the examples above.

The need for this additional twist in the definition is best understood with an example. Consider two atomic types $A$ and $B$ and the term $(\lambda x: A \times B \cdot x) z:$ if $k$ is fixed to be one (i.e. we allow only one expansor as marker) then its translation $((\lambda x: A \times B . x) z)^{\circ}$ is $\Delta_{A \times B}\left(\left(\lambda x: A \times B . \Delta_{A \times B} x\right) \Delta_{A \times B} z\right)$. Now $(\lambda x: A \times B . x) z \stackrel{\leftrightarrow}{\longrightarrow}$, so we have to verify that $((\lambda x: A \times B \cdot x) z)^{\circ}$ reduces to $z^{\circ}$ in at least one step. We have:

$$
\Delta_{A \times B}\left(\left(\lambda x: A \times B \cdot \Delta_{A \times B} x\right) \Delta_{A \times B} z\right) \Longrightarrow \Delta_{A \times B} \Delta_{A \times B} \Delta_{A \times B} z
$$

However, even if both $\Delta_{A \times B}^{3} z$ and $\Delta_{A \times B} z$ reduce to the same term $\left\langle\pi_{1}(z), \pi_{2}(z)\right\rangle$, it is not true that $\Delta_{A \times B}^{3} z \Longrightarrow * \Delta_{A \times B} z$. Anyway, if we admit $\Delta_{A \times B}^{3} z$ as a possible translation of $z$ we will have the desired property relating reductions and translations. Hence, to be precise, our method associates to each term not just one translation, but a whole family of possible translations, all with the same structure, but with different numbers of expansors used as markers.

What is important for our proof is that when we are given a reduction $M_{1} \Longrightarrow M_{2} \ldots \Longrightarrow M_{n}$ in the full calculus, then no matter which possible translation $M_{1}^{0}$ we choose for $M_{1}$, the reductions used in the simulation process all go through possible translations $M_{i}^{0}$ of the $M_{i}$.

Translations preserve types and leave unchanged terms where expansions are not possible.

\footnotetext{
${ }^{5}$ Notice that we cannot insert expansors in influential positions: if a term $M$ is expanded, say to $\left\langle\pi_{1}(M), \pi_{2}(M)\right\rangle$, then its root becomes an influential position, and we cannot insure that the translation of $M$ reduces to a translation of $\left\langle\pi_{1}(M), \pi_{2}(M)\right\rangle$ : expansors get used, and after some reduction steps we end up with a naked pair not preceded by an expansor.
} 
Lemma 5.3 If I' $M: A$, then $\Gamma \vdash\left(\Delta_{A} M\right): A$.

Proof. By induction on the structure of $A$.

- If $A$ is neither a functional, nor a product type, then $\Delta_{A}$ is empty and the property trivially holds.

- $A \equiv B \rightarrow C$. Since $\Gamma, x: B \rightarrow C, z: B \vdash z: B$, we have by induction hypothesis $\Gamma, x: B \rightarrow$ $C, z: B \vdash\left(\Delta_{B} z\right): B$

$$
\frac{\Gamma, x: B \rightarrow C, z: B \vdash x: B \rightarrow C \quad \Gamma, x: B \rightarrow C, z: B \vdash\left(\Delta_{B} z\right): B}{\Gamma, x: B \rightarrow C, z: B \vdash\left(x\left(\Delta_{B} z\right)\right): C}
$$

Again by induction hypothesis $\Gamma, x: B \rightarrow C, z: B \vdash \Delta_{C}\left(x\left(\Delta_{B} z\right)\right): C$ and thus:

$$
\frac{\frac{\Gamma, x: B \rightarrow C, z: B \vdash \Delta_{C}\left(x\left(\Delta_{B} z\right)\right): C}{\Gamma, x: B \rightarrow C \vdash \lambda z: B \cdot \Delta_{C}\left(x\left(\Delta_{B} z\right)\right): B \rightarrow C}}{\Gamma \vdash \lambda x: B \rightarrow C \cdot \lambda z: B \cdot \Delta_{C}\left(x\left(\Delta_{B} z\right)\right):(B \rightarrow C) \rightarrow(B \rightarrow C)} \quad \Gamma \vdash M: B \rightarrow C
$$

- $A \equiv B \times C$. Since $\Gamma, x: B \times C \vdash x: B \times C$, then $\Gamma, x: B \times C \vdash \pi_{1}(x): B$ and $\Gamma, x: B \times C \vdash$ $\pi_{2}(x): C$. By induction hypothesis $\Gamma, x: B \times C \vdash \Delta_{B} \pi_{1}(x): B$ and $\Gamma, x: B \times C \vdash \Delta_{C} \pi_{1}(x): C$.

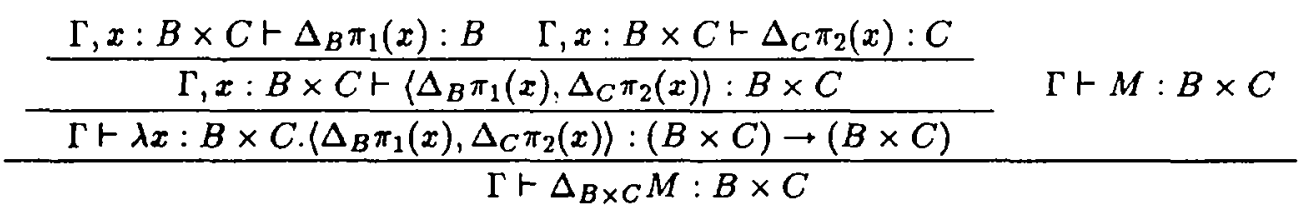

$\square$

Corollary 5.4 If $\Gamma \vdash M: A$, then $\Gamma \vdash \Delta_{A}^{k} M: A$, for any $k \geq 0$.

Lemma 5.5 (Type Preservation) If $\Gamma \vdash M: A$, then $\Gamma \vdash M^{\circ}: A$ and $\Gamma \vdash M^{\circ 0}: A$.

Proof. By induction on the structure of $M$, using corollary 5.4 .

$\square$

A term $M$ is in quasi-normal form if only expansion rules at the root position are applicable to it and $M$ is in normal form if no rule is applicable to it. So, every normal form is in quasi-normal form, while the converse does not necessarily hold.

\section{Lemma 5.6}

1. If $M$ is in normal form, then $M^{\circ}=M$

2. If $M$ is in quasi-normal form, then $M^{\circ 0}=M$

Proof. By induction on the structure of $M$.

- $M \equiv *$.

1. $*^{\circ}=*$.

2. The property vacuously holds because $*$ is a normal form.

- $M \equiv x$. 
1. Since $\boldsymbol{x}$ is in normal form, it has neither a functional, nor a product, nor the $\mathbf{T}$ type and then $\Delta_{A}$ is empty, where $A$ is the type of $x$. Then $x^{\circ}=x$.

2. $x^{\circ 0}=x$ by definition.

- $M \equiv \lambda x: A . P$.

1. Since $M$ is in normal form, $P$ is also in normal form and by induction hypothesis $P^{\circ}=P$. We have $(\lambda x: A . P)^{\circ}=\lambda x: A . P^{\circ}=\lambda x: A . P$.

2. If $\lambda x: A . P$ is in quasi-normal form, it is also in normal form because we cannot apply an expansion rule to a lambda-term. By the previous paragraph $(\lambda x: A . P)^{\circ 0}=\lambda x: A . P$.

- $M \equiv\langle P, Q\rangle$.

1. Since $M$ is in normal form, $P$ and $Q$ are also in normal form and by induction hypothesis $P^{\circ}=P$ and $Q^{\circ}=Q$. We have $\langle P, Q\rangle^{\circ}=\left\langle P^{\circ}, Q^{\circ}\right\rangle=\langle P, Q\rangle$.

2. If $\langle P, Q\rangle$ is in quasi-normal form, it is also in normal form because we cannot apply an expansion rule to a pair. By the previous paragraph $\langle P, Q\rangle^{\circ 0}=\langle P, Q\rangle$.

- $M \equiv(\operatorname{rec} y: A . P)^{i}$.

- If $i=0$, then

1. Since $M$ is in normal form, $P$ is also in normal form and by induction hypothesis $P^{\circ}=P$. On the other hand, $M$ has neither a functional, nor a product, nor the $\mathrm{T}$ type and then $\Delta_{A}$ is empty, where $A$ is the type of $M$. We have $(\text { rec } y: A . P)^{0^{\circ}}=$ $\Delta_{A}^{k}\left(\operatorname{rec} y: A . P^{0}\right)^{0}=(\text { rec } y: A . P)^{0}$.

2. Since $M$ is in quasi normal form, $P$ is in normal form and by induction hypothesis $P^{\circ}=P$. Then $(\operatorname{rec} y: A . P)^{0^{\circ}}=\left(\operatorname{rec} y: A . P^{\circ}\right)^{0}=(\operatorname{rec} y: A . P)^{0}$.

- If $i>0$, then

1. The property vacuously holds because (rec $y: A . P)^{i}$ is not in normal form.

2. The property vacuously holds because $(\operatorname{rec} y: A . P)^{i}$ is not in quasi-normal form.

- $M \equiv(P Q)$.

1. Suppose $A$ is the type of $M$. Since $M$ is in normal form, $A$ is neither a functional, nor a product, nor the $\mathbf{T}$ type and so $\Delta_{A}$ is empty. On the other hand $P$ is in quasi-normal form and $Q$ is in normal form, so by induction hypothesis $P^{\circ \circ}=P$ and $Q^{\circ}=Q$. We have $(P Q)^{\circ}=\Delta_{A}^{k}\left(P^{00} Q^{\circ}\right)=(P Q)$.

2. Since $M$ is in quasi-normal form, $P$ is in quasi-normal form and $Q$ is in normal form and by induction hypothesis $P^{\circ \circ}=P$ and $Q^{\circ}=Q$. We have $(P Q)^{\circ \circ}=\left(P^{\circ \circ} Q^{\circ}\right)=(P Q)$.

- $M \equiv \operatorname{Case}(P, R, N)$.

1. Suppose $A$ is the type of $M$. Since $M$ is in normal form, $A$ is neither a functional, nor a product, nor the $\mathbf{T}$ type and so $\Delta_{A}$ is empty. On the other hand $P, R$ and $N$ are in normal form and by induction hypothesis $P^{\circ}=P$ and $R^{\circ}=R$ and $N^{\circ}=R$. We have $\operatorname{Case}(P, R, N)^{\circ}=\Delta_{A}^{k} \operatorname{Case}\left(P^{\circ}, R^{\circ}, N^{\circ}\right)=\operatorname{Case}(P, R, N)$.

2. Since $M$ is in quasi-normal form, $P, R$ and $N$ are in normal form and by induction hypothesis $P^{\circ}=P$ and $R^{\circ}=R$ and $N^{\circ}=R$. We have $\operatorname{Case}(P, R, N)^{\circ \circ}=\operatorname{Case}\left(P^{\circ}, R^{\circ}, N^{\circ}\right)=$ $\operatorname{Case}(P, R, N)$.

- $M \equiv \pi_{i}(P)$, for $i=1,2$.

1. Suppose $A$ is the type of $M$. Since $M$ is in normal form, $A$ is neither a functional, nor a product, nor the $\mathrm{T}$ type and so $\Delta_{A}$ is empty. On the other hand $P$ is in quasi-normal form and by induction hypothesis $P^{\circ \circ}=P$. We have $\pi_{i}(P)^{\circ}=\Delta_{A}^{k} \pi_{i}\left(P^{\circ 0}\right)=\pi_{i}(P)$. 
2. Since $M$ is in quasi-normal form, $P$ is also in quasi-normal form and by induction hypothesis $P^{\circ 0}=P$. We have $\pi_{i}(P)^{\circ \circ}=\pi_{i}\left(P^{\circ 0}\right)=\pi_{i}(P)$.

- $M \equiv i n_{C}^{i}(P)$, for $i=1,2$.

1. Since $M$ is in normal form, $P$ is also in normal form and by induction hypothesis $P^{\circ}=P$. We have $i n_{C}^{i}(P)^{\circ}=i n_{C}^{i}\left(P^{\circ}\right)=i n_{C}^{i}(P)$.

2. $i n_{C}^{i}(P)$ in quasi-normal form implies $i n_{C}^{i}(P)$ in normal form, and the property holds by the previous paragraph.

The next step is to prove that we can apply proposition 5.1 to our system, i.e, for every one step reduction from $M$ to $N$ in the full system, there is a non empty reduction sequence in the system without expansions from any translation of $M$ to a translation of $N$.

This lemma characterizes the reductions from a term $\Delta_{A \rightarrow B}^{k} M$ or $\Delta_{A \times B}^{k} M$ and is quite essential in all the properties shown in this section.

Lemma 5.7 For any $k>0$

$$
\Delta_{A \rightarrow B}^{k} M \Longrightarrow+\lambda w: A \cdot \Delta_{B}^{k}\left(M\left(\Delta_{A}^{k} w\right)\right)
$$

and

$$
\Delta_{A \times B}^{k} M \Longrightarrow+\left\langle\Delta_{A}^{k} \pi_{1}(M), \Delta_{B}^{k} \pi_{2}(M)\right\rangle
$$

and the reduction sequences contain no expansion steps.

Proof. By induction on $k$.

If $k=1$, then

$$
\begin{aligned}
& \Delta_{A \rightarrow B} M \equiv\left(\lambda x: A \rightarrow B \cdot \lambda w: A \cdot \Delta_{B}\left(x\left(\Delta_{A} w\right)\right)\right) M \stackrel{\rho}{\longrightarrow} \lambda w: A \cdot \Delta_{B}\left(M\left(\Delta_{A} w\right)\right) \\
& \Delta_{A \times B} M \equiv\left(\lambda x: A \times B \cdot\left(\Delta_{A} \pi_{1}(x), \Delta_{B} \pi_{2}(x)\right)\right) M \stackrel{\rho}{\longrightarrow}\left\langle\Delta_{A} \pi_{1}(M), \Delta_{B} \pi_{2}(M)\right)
\end{aligned}
$$

Only the $\beta$-rule is used in this reduction.

If $k>1$, then

$$
\begin{aligned}
& \Delta_{A \rightarrow B}^{k+1} M \\
& = \\
& \Delta_{A \rightarrow B}^{k} \Delta_{A \rightarrow B} M
\end{aligned}
$$

$\Downarrow_{\beta}$

$$
\Delta_{A \rightarrow B}^{k} \lambda w: A \cdot \Delta_{B}\left(M\left(\Delta_{A} w\right)\right)
$$

$\Downarrow_{+}$by induction hypothesis (and without expansions steps

$$
\lambda w: A \cdot \Delta_{B}^{k}\left(\lambda w: A \cdot \Delta_{B}\left(M\left(\Delta_{A} w\right)\right)\left(\Delta_{A}^{k} w\right)\right)
$$

$\Downarrow_{B}$

$$
\begin{aligned}
& \lambda w: A \cdot \Delta_{B}^{k}\left(\Delta_{B}\left(M\left(\Delta_{A} \Delta_{A}^{k} w\right)\right)\right) \\
& =\quad \\
& \lambda w: A \cdot \Delta_{B}^{k+1}\left(M\left(\Delta_{A}^{k+1} w\right)\right)
\end{aligned}
$$




$$
\begin{aligned}
& \Delta_{A \times B}^{k+1} M \\
& ={ }^{k}{ }_{A \times B}\left(\Delta_{A \times B} M\right)
\end{aligned}
$$

$\Downarrow_{B}$

$$
\Delta_{A \times B}^{k}\left\langle\Delta_{A} \pi_{1}(M), \Delta_{B} \pi_{2}(M)\right\rangle
$$

$\Downarrow_{+}$by induction hypothesis (and without expansion steps)

$$
\begin{aligned}
& \left\langle\Delta_{A}^{k} \pi_{1}\left(\left\langle\Delta_{A} \pi_{1}(M), \Delta_{B} \pi_{2}(M)\right\rangle\right), \Delta_{B}^{k} \pi_{2}\left(\left\langle\Delta_{A} \pi_{1}(M), \Delta_{B} \pi_{2}(M)\right\rangle\right)\right\rangle \\
& \Downarrow_{\pi_{1}, \pi_{2}} \\
& \left\langle\Delta_{A}^{k} \Delta_{A} \pi_{1}(M), \Delta_{B}^{k} \Delta_{B} \pi_{2}(M)\right\rangle \\
& = \\
& \left\langle\Delta_{A}^{k+1} \pi_{1}(M), \Delta_{B}^{k+1} \pi_{2}(M)\right\rangle
\end{aligned}
$$

We use $N^{\otimes}$ to denote either $N^{\circ}$ or $N^{\circ 0}$. In particular, $\overline{N^{\otimes}}$ will stand for a sequence of mixed $N_{i}^{\circ}$ 's and $N_{i}^{\circ \circ}$ 's.

Lemma 5.8 If $\Gamma \vdash M: A$, then

1. $\exists k \geq 0, M^{00}\left[\overline{z^{\otimes}} / \bar{x}\right] \Longrightarrow^{*} \Delta_{A}^{k}(M[\bar{z} / \bar{x}])^{\circ 0}$

2. $\forall k \geq 0, \Delta_{A}^{k} M^{\circ}\left[\overline{z^{\otimes}} / \bar{x}\right] \Longrightarrow^{*}(M[\bar{z} / \bar{x}])^{\circ}$

and no expansions are performed in these reduction sequences.

Proof. We show the two properties by induction on the structure of $M$. More precisely, for the first statement we analyze each case, while for the second one it is enough to analyze those expressions $M$ such that $M^{\circ}=M^{\circ \circ}$. Indeed, once we have already shown the first statement, the second can be easily shown in the following way for the expressions $M$ such that $M^{\circ}=\Delta_{A}^{h} M^{\circ \circ}$ (for $h>0$ ):

$$
\begin{aligned}
& \Delta_{A}^{k} M^{\circ}\left[\overline{z^{\otimes}} / \bar{x}\right]=\text { for some } h>0 \\
& \Delta_{A}^{k} \Delta_{A}^{h} M^{\circ 0}\left[\overline{z^{\otimes}} / \bar{x}\right] \Longrightarrow^{*} \text { by the first statement } \\
& \Delta_{A}^{k+h} \Delta_{A}^{m} M[\bar{z} / \bar{x}]^{\circ o}= \\
& M[\bar{z} / \bar{x}]^{\circ}, \text { because } h>0
\end{aligned}
$$

Every reduction built in the following proof contains no expansion steps, as it is constructed from one-step reductions that are not expansions or from reductions obtained by induction hypothesis (and thus without expansions) or from reductions obtained by lemma 5.7 (again without expansions). This remark will allow us to conclude that the reductions in the statements of the lemma contain no expansion.

Now, let us analyze the first statement and the interesting cases of the second.

- $M \equiv *$. Since $*$ is of type $\mathrm{T}, \Delta_{\mathbf{T}}$ is empty. $*^{00}\left[\overline{z^{\otimes}} / \bar{x}\right]=*\left[\overline{z^{\otimes}} / \bar{x}\right]=*=*^{00}=\Delta_{\mathbf{T}^{* 00}}^{0}=$ $\Delta_{\mathrm{T}}^{0}(*[\bar{z} / \bar{x}])^{00}$.

- $M \equiv x_{i} \in \bar{x}$.

$$
\begin{aligned}
& -x_{i}^{0 \circ}\left[\overline{z^{\otimes}} / \bar{x}\right]=x_{i}\left[\overline{z^{\otimes}} / \bar{x}\right]=z_{i}^{0}=\Delta_{A}^{m} z_{i}^{00}=\Delta_{A}^{m}\left(x_{i}[\bar{z} / \bar{x}]\right)^{\circ 0} . \\
& -x_{i}^{\circ \circ}\left[\overline{z^{\otimes}} / \bar{x}\right]=x_{i}\left[\overline{z^{\otimes}} / \bar{x}\right]=z_{i}^{00}=\left(x_{i}[\bar{z} / \bar{x}]\right)^{\circ 0}=\Delta_{A}^{0}\left(x_{i}[\bar{z} / \bar{x}]\right)^{\circ 0} .
\end{aligned}
$$


- $M \equiv y \notin \bar{x}$.

$y^{\circ 0}\left[\overline{z^{\otimes}} / \bar{x}\right]=y\left[\overline{z^{\otimes}} / \bar{x}\right]=y=\Delta_{A}^{0} y^{00}$.

- $M \equiv(P Q)$

$(P Q)^{\circ 0}\left[\overline{z^{\otimes}} / \bar{x}\right]=\left(P^{\circ 0} Q^{\circ}\right)\left[\overline{z^{\otimes}} / \bar{x}\right]=P^{00}\left[\overline{z^{\otimes}} / \bar{x}\right] Q^{\circ}\left[\overline{z^{\otimes}} / \bar{x}\right]$.

By induction hypothesis $P^{\circ \circ}\left[\overline{z^{\otimes}} / \bar{x}\right] \Longrightarrow^{*} \Delta_{B \rightarrow A}^{h} P[\bar{z} / \bar{x}]^{00}$

- If $h=0$, then

$P^{\circ 0}\left[\overline{z^{\otimes}} / \bar{x}\right] Q^{\circ}\left[\overline{z^{\otimes}} / \bar{x}\right]$

ฟ. by induction hypothesis

$P[\bar{z} / \bar{x}]^{\circ \circ} Q^{\circ}\left[\overline{z^{\otimes}} / \bar{x}\right]$

ฟ. by induction hypothesis

$$
\begin{aligned}
& P[\bar{z} / \bar{x}]^{\circ 0} Q[\bar{z} / \bar{x}]^{\circ} \\
& = \\
& (P[\bar{z} / \bar{x}] Q[\bar{z} / \bar{x}])^{\circ 0} \\
& = \\
& ((P Q)[\bar{z} / \bar{x}])^{\circ 0} \\
& = \\
& \Delta_{A}^{0}(P Q[\bar{z} / \bar{x}])^{\circ 0}
\end{aligned}
$$

- If $h>0$, then:

$\Delta_{B \rightarrow A}^{h} P[\bar{z} / \bar{x}]^{\circ 0} Q^{\circ}\left[\overline{z^{8}} / \bar{x}\right]$

$\Downarrow_{+}$by lemma 5.7

$\left(\lambda w: B \cdot \Delta_{A}^{h}\left(P[\bar{z} / \bar{x}]^{\circ \circ}\left(\Delta_{B}^{h} w\right)\right)\right) Q^{\circ}\left[\overline{z^{8}} / \bar{x}\right]$

$\Downarrow_{\beta}$

$\Delta_{A}^{h}\left(P[\bar{z} / \bar{x}]^{\circ 0}\left(\Delta_{B}^{h}\left(Q^{\circ}\left[\overline{z^{\otimes}} / \bar{x}\right]\right)\right)\right)$

$\Downarrow_{*}$ by induction hypothesis

$\Delta_{A}^{h}\left(P[\bar{z} / \bar{x}]^{\circ \circ} Q[\bar{z} / \bar{x}]^{\circ}\right)$

$=$

$\Delta_{A}^{h}(P[\bar{z} / \bar{x}] Q[\bar{z} / \bar{x}])^{\circ 0}$

$\bar{\Delta}_{A}^{h}(P Q[\bar{z} / \bar{x}])^{\circ 0}$

- $M \equiv \lambda y: B . P$. 
1.

$$
\begin{aligned}
& (\lambda y: B \cdot P)^{\circ \circ}\left[\overline{z^{\otimes}} / \bar{x}\right] \\
& = \\
& \left(\lambda y: B \cdot P^{\circ}\right)\left[\overline{z^{\otimes}} / \bar{x}\right] \\
& = \\
& \lambda y: B \cdot P^{\circ}\left[\overline{z^{\otimes}} / \bar{x}\right] \\
& \Downarrow \cdot \quad \text { by induction hypothesis } \\
& \lambda y: B \cdot P[\bar{z} / \bar{x}]^{\circ} \\
& = \\
& (\lambda y: B \cdot P[\bar{z} / \bar{x}])^{\circ 0} \\
& = \\
& \Delta_{B \rightarrow C}^{0}((\lambda y: B \cdot P)[\bar{z} / \bar{x}])^{\circ 0}
\end{aligned}
$$

2.

$$
\begin{aligned}
& \Delta_{B \rightarrow C}^{k}(\lambda y: B \cdot P)^{\circ}\left[\overline{z^{\otimes}} / \bar{x}\right] \\
& =\overline{\Delta_{B \rightarrow C}^{k}}\left(\lambda y: B \cdot P^{\circ}\right)\left[\overline{z^{\otimes}} / \bar{x}\right] \\
& = \\
& \Delta_{B \rightarrow C}^{k} \lambda y: B \cdot P^{\circ}\left[\overline{z^{\otimes}} / \bar{x}\right]
\end{aligned}
$$

$\Downarrow_{+}$by lemma 5.7

$\lambda w: B . \Delta_{B}^{k}\left(\left(\lambda y: B \cdot P^{\circ}\left[\overline{z^{\otimes}} / \bar{x}\right]\right)\left(\Delta_{C}^{k} w\right)\right)$

$\Downarrow_{B}$

$\lambda w: B \cdot \Delta_{B}^{k} P^{\circ}\left[\overline{z^{\otimes}} / \bar{x}\right]\left[w^{\circ} / y\right]$

V. by induction hypothesis

$\lambda w: B \cdot(P[\bar{z} / \bar{x}][w / y])^{\circ}$

$=$

$(\lambda w: B . P[\bar{z} / \bar{x}][w / y])^{\circ}$

$=\alpha$

$(\lambda y: B \cdot P[\bar{z} / \bar{x}])^{\circ}$

$=$

$((\lambda y: B . P)[\bar{z} / \bar{x}])^{\circ}$

- $M \equiv \pi_{i}(P)$, for $i=1,2$.

$$
\begin{aligned}
& \pi_{i}(P)^{00}\left[\overline{z^{\otimes}} / \bar{x}\right] \\
& = \\
& \pi_{i}\left(P^{00}\right)\left[\overline{z^{\otimes}} / \bar{x}\right] \\
& = \\
& \pi_{i}\left(P^{00}\left[\overline{z^{\otimes}} / \bar{x}\right]\right)
\end{aligned}
$$

ฟ. by induction hypothesis

$$
\pi_{i}\left(\Delta_{A_{1} \times A_{2}}^{h}\left(P[\bar{z} / \bar{x}]^{00}\right)\right.
$$


- If $h=0$, then

$$
\begin{aligned}
& \pi_{i}\left(P[\bar{z} / \bar{x}]^{\circ 0}\right) \\
& = \\
& \pi_{i}(P[\bar{z} / \bar{x}])^{\circ 0} \\
& = \\
& \Delta_{A_{i}}^{0} \pi_{i}(P[\bar{z} / \bar{x}])^{\circ 0} \\
& =\overline{\Delta_{A_{i}}^{0}\left(\pi_{i}(P)[\bar{z} / \bar{x}]\right)^{\circ 0}}
\end{aligned}
$$

- If $h>0$, then

$$
\pi_{i}\left(\Delta_{A_{1} \times A_{2}}^{h}(P[\bar{z} / \bar{x}])^{\circ 0}\right)
$$

$\Downarrow_{+}$by lemma 5.7

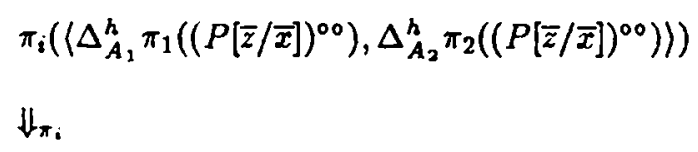

- $M \equiv i n_{C}^{i}(P)$, for $i=1,2$ and then $\Delta_{A}$ is empty.

$$
\begin{aligned}
& \operatorname{in}_{C}^{i}(P)^{\circ 0}\left[\overline{z^{\otimes}} / \bar{x}\right] \\
& = \\
& i n_{C}^{i}\left(P^{\circ}\right)\left[\overline{z^{\otimes}} / \bar{x}\right] \\
& = \\
& i n_{C}^{i}\left(P^{\circ}\left[\overline{z^{\otimes}} / \bar{x}\right]\right) \\
& \text { W. by induction hypothesis } \\
& \text { in } \\
& = \\
& \Delta_{A}^{i}\left(P[\bar{z} / \bar{x}]_{C}^{\circ}\right) \\
& = \\
& \left.\left.\Delta_{A}^{0}(P[\bar{z} / \bar{x}])_{C}^{i}(P)[\bar{z}] / \bar{x}\right]\right)^{\circ 0}
\end{aligned}
$$

- $M \equiv\langle P, Q\rangle$. 
1.

$$
\begin{aligned}
& \langle P, Q\rangle^{\circ \circ}\left[\overline{z^{\otimes}} / \bar{x}\right] \\
& = \\
& \left\langle P^{\circ}, Q^{\circ}\right\rangle\left[\overline{z^{\otimes}} / \bar{x}\right] \\
& == \\
& \left\langle P^{\circ}\left[\overline{z^{\otimes}} / \bar{x}\right], Q^{\circ}\left[\overline{z^{\otimes}} / \bar{x}\right]\right\rangle
\end{aligned}
$$

W* by induction hypothesis

$$
\begin{aligned}
& \left\langle P[\bar{z} / \bar{x}]^{0}, Q[\bar{z} / \bar{x}]^{0}\right\rangle \\
& = \\
& \langle P[\bar{z} / \bar{x}], Q[\bar{z} / \bar{x}]\rangle^{\circ 0} \\
& = \\
& \Delta_{A \times B}^{0}\langle P[\bar{z} / \bar{x}], Q[\bar{z} / \bar{x}]\rangle^{\circ 0} \\
& ={ }_{\Delta_{A \times B}^{0}}(\langle P, Q\rangle[\bar{z} / \bar{x}])^{\circ 0}
\end{aligned}
$$

2.

$$
\begin{aligned}
& \Delta_{A \times B}^{k}\langle P, Q\rangle^{\circ}\left[\overline{z^{\otimes}} / \bar{x}\right] \\
& = \\
& \Delta_{A \times B}^{k}\left\langle P^{\circ}, Q^{\circ}\right\rangle\left[\overline{z^{\otimes}} / \bar{x}\right] \\
& = \\
& \Delta_{A \times B}^{k}\left\langle P^{\circ}\left[\overline{z^{\otimes}} / \bar{x}\right], Q^{\circ}\left[\overline{z^{\otimes}} / \bar{x}\right]\right)
\end{aligned}
$$

- If $k=0$, then

$$
\left\langle P^{\circ}\left[\overline{z^{\otimes}} / \bar{x}\right], Q^{\circ}\left[\overline{z^{\otimes}} / \bar{x}\right]\right\rangle
$$

$\Downarrow$. by induction hypothesis

$$
\begin{aligned}
& \left\langle P[\bar{z} / \bar{x}]^{\circ}, Q[\bar{z} / \bar{x}]^{\circ}\right\rangle \\
& = \\
& \langle P[\bar{z} / \bar{x}], Q[\bar{z} / \bar{x}]\rangle^{\circ} \\
& = \\
& (\langle P, Q\rangle[\bar{z} / \bar{x}])^{\circ}
\end{aligned}
$$

- If $k>0$, then

$\Delta_{A \times B}^{k}\left\langle P^{\circ}\left[\overline{z^{\otimes}} / \bar{x}\right], Q^{\circ}\left[\overline{z^{\otimes}} / \bar{x}\right]\right\rangle$

$\Downarrow_{+}$by lemma 5.7

$\left\langle\Delta_{A}^{k} \pi_{1}\left(P^{\circ}\left[\overline{z^{\otimes}} / \bar{x}\right] Q^{\circ}\left[\overline{z^{\otimes}} / \bar{x}\right]\right), \Delta_{B}^{k} \pi_{2}\left(\left\langle P^{\circ}\left[\overline{z^{\otimes}} / \bar{x}\right], Q^{\circ}\left[\overline{z^{\otimes}} / \bar{x}\right]\right\rangle\right)\right\rangle$

$\Downarrow \pi_{1}, \pi_{2}$

$\left\langle\Delta_{A}^{k} P^{\circ}\left[\overline{z^{\otimes}} / \bar{x}\right], \Delta_{B}^{k} Q^{\circ}\left[\overline{z^{\otimes}} / \bar{x}\right]\right\rangle$

W. by induction hypothesis

$$
\begin{aligned}
& \left\langle P[\bar{z} / \bar{x}]^{\circ}, Q[\bar{z} / \bar{x}]^{\circ}\right\rangle \\
& = \\
& \langle P[\bar{z} / \bar{x}], Q[\bar{z} / \bar{x}]\rangle^{\circ} \\
& = \\
& (\langle P, Q\rangle[\bar{z} / \bar{x}])^{\circ}
\end{aligned}
$$


- $M \equiv(\operatorname{rec} y: A . P)^{i}$

$$
\begin{aligned}
& (\operatorname{rec} y: A . P)^{i \circ 0}\left[\overline{z^{\otimes}} / \bar{x}\right] \\
& =\left(\operatorname{rec} y: A . P^{0}\right)^{i}\left[\overline{z^{\otimes}} / \bar{x}\right]
\end{aligned}
$$

$\Downarrow$. by induction hypothesis

$$
\begin{aligned}
& \left(\operatorname{rec} y: A \cdot(P[\bar{z} / \bar{x}])^{\circ}\right)^{i} \\
& = \\
& \Delta_{A}^{0}(\operatorname{rec} y: A \cdot P[\bar{z} / \bar{x}])^{i 00} \\
& == \\
& \Delta_{A}^{0}\left((\operatorname{rec} y: A \cdot P)^{i}[\bar{z} / \bar{x}]\right)^{\circ \circ}
\end{aligned}
$$

- $M \equiv \operatorname{Case}(P, Q, R)$

$$
\begin{aligned}
& \operatorname{Case}(P, Q, R)^{\circ \circ}\left[\overline{z^{\otimes}} / \bar{x}\right] \\
& = \\
& \operatorname{Case}\left(P^{\circ}, Q^{\circ}, R^{\circ}\right)\left[\overline{z^{\otimes}} / \bar{x}\right] \\
& = \\
& \operatorname{Case}\left(P^{\circ}\left[\overline{z^{\otimes}} / \bar{x}\right], Q^{\circ}\left[\overline{z^{\otimes}} / \bar{x}\right], R^{\circ}\left[\overline{z^{\otimes}} / \bar{x}\right]\right)
\end{aligned}
$$

W. by induction hypothesis

$$
\begin{aligned}
& \operatorname{Case}\left(P[\bar{z} / \bar{x}]^{\circ}, Q[\bar{z} / \bar{x}]^{\circ}, R[\bar{z} / \bar{x}]^{\circ}\right) \\
& = \\
& \operatorname{Case}(P[\bar{z} / \bar{x}], Q[\bar{z} / \bar{x}], R[\bar{z} / \bar{x}])^{\circ \circ} \\
& = \\
& (\operatorname{Case}(P, Q, R)[\bar{z} / \bar{x}])^{\circ 0} \\
& = \\
& \Delta_{A}^{0}(\operatorname{Case}(P, Q, R)[\bar{z} / \bar{x}])^{\circ \circ}
\end{aligned}
$$

Corollary 5.9 If $\Gamma \vdash M: A$, then

1. $\forall k \geq 0, \Delta_{A}^{k} M^{\circ} \Longrightarrow^{*} M^{\circ}$

2. $\exists k \geq 0, M^{\circ 0} \Longrightarrow^{*} \Delta_{A}^{k} M^{\circ 0}$

and no expansions are performed in the reduction sequences.

The following property is essential to show that every time we perform a $\beta$-reduction on a term $M$ in the original system, any translation of $M$ reduces to a translation of the term we have obtained via $\rightarrow \beta$ from $M$. Take for example the reduction $(\lambda x: A . M) N \rightarrow_{\beta} M[N / x]$. We know that $((\lambda x:$ $A \cdot M) N)^{\circ}=\Delta_{A}^{k}\left(\left(\lambda x: A \cdot M^{\circ}\right) N^{\circ}\right)$ and we want to show that there is a non empty reduction sequence leading to $M[N / x]^{\circ}$. Since $\Delta_{A}^{k}\left(\left(\lambda x: A \cdot M^{\circ}\right) N^{\circ}\right) \rightarrow_{\beta} \Delta_{A}^{k} M^{\circ}\left[N^{\circ} / x\right]$, we have now to check that the term $\left(M[N / 2:)^{\circ}\right.$ can be reached. We state the property as follows:

Lemma 5.10 If $\Gamma \vdash M: A$, then

1. $\forall k \geq 0, \Delta_{A}^{k} M^{\circ}\left[\overline{N^{\circ}} / \bar{x}\right] \Longrightarrow^{*}(M[\bar{N} / \bar{x}])^{\circ}$

2. $\exists k \geq 0, M^{\circ 0}\left[\overline{N^{\circ}} / \bar{x}\right] \Longrightarrow^{*} \Delta_{A}^{k}(M[\bar{N} / \bar{x}])^{\circ \circ}$

and no expansions are performed in the reduction sequences. 
Proof. The proof of this lemma can be done exactly as for lemma 5.8, except for the case $M \equiv x_{i}$, where to prove the first statement we need to use corollary 5.9 as follows:

$\Delta_{A}^{k} x_{i}^{0}\left[\overline{N^{\circ}} / \bar{x}\right]=\Delta_{A}^{k}\left(\Delta_{A}^{m} x_{i}\right)\left[\overline{N^{0}} / \bar{x}\right]=\Delta_{A}^{k+m} N_{i}^{0}$.

By corollary $5.9, \Delta_{A}^{k+m} N_{i}^{\circ} \Longrightarrow * N_{i}^{\circ}=\left(x_{i}[\bar{N} / \bar{x}]\right)^{\circ}$.

Notice that there are no expansions in the sequence $\Delta_{A}^{k+m} N_{i}^{0} \Rightarrow^{*} N_{i}^{0}$ by corollary 5.9 .

Lemma 5.11 If $\Gamma \vdash M: A$, then

1. $\forall k \geq 0, \Delta_{A}^{k} M^{\circ}\left[\overline{N^{00}} / \bar{x}\right] \Rightarrow^{*}(M[\bar{N} / \bar{x}])^{\circ}$

2. $\exists k \geq 0, M^{\circ 0}\left[\overline{N^{\circ 0}} / \bar{x}\right] \Longrightarrow^{*} \Delta_{A}^{k}(M[\bar{N} / \bar{x}])^{\circ 0}$

and no expansions are performed in the reduction sequences.

Proof. The proof of this lemma can be done exactly as for lemma 5.8, except for the case $M \equiv x_{i}$, where to prove the first statement we need to use corollary 5.9 as follows:

$\Delta_{A}^{k} x_{i}^{\circ}\left[\overline{N^{\circ 0}} / \bar{x}\right]=\Delta_{A}^{k}\left(\Delta_{A}^{m} x_{i}\right)\left[\overline{N^{\circ 0}} / \bar{x}\right]=\Delta_{A}^{k+m} N_{i}^{\circ 0}$.

If $N_{i}^{\circ}=N_{i}^{\circ 0}$, then by corollary $5.9 \Delta_{A}^{k+m} N_{i}^{\circ} \Longrightarrow * N_{i}^{\circ}=\left(x_{i}[\bar{N} / \bar{x}]\right)^{\circ}$.

In the other case $\Delta_{A}^{k+m} N_{i}^{\circ 0}=N_{i}^{0}=\left(x_{i}[\bar{N} / \bar{x}]\right)^{\circ}$.

Notice that there are no expansions in the sequence $\Delta_{A}^{k+m} N_{i}^{o} \Longrightarrow N_{i}^{o}$ by corollary 5.9 .

口

Lemma 5.12 If $M \stackrel{n, 6, T_{0},}{\longrightarrow} N$, then $M^{\sigma_{0,-} n+} N^{\circ}$.

Proof.

- If $M$ is of type $A \times B$ and $M \stackrel{\delta}{\longrightarrow}\left\langle\pi_{1}(M), \pi_{2}(M)\right\rangle$

We know that $\exists k>0$ such that $M^{\circ}=\Delta_{A \times B}^{k} M^{00}$. By corollary 5.7

$$
\Delta_{A \times B}^{k} M^{00} \Longrightarrow+\left(\Delta_{A}^{k} \pi_{1}\left(M^{00}\right), \Delta_{B}^{k} \pi_{2}\left(M^{00}\right)\right)
$$

and the sequence has no expansion rules.

The last term is equal to $\left\langle\pi_{1}(M)^{\circ}, \pi_{2}(M)^{\circ}\right\rangle=\left\langle\pi_{1}(M), \pi_{2}(M)\right\rangle^{\circ}$ and then the property holds.

- If $M$ is of type $A \rightarrow B$ and $M \stackrel{n}{\longrightarrow} \lambda y: A . M y$

We know that $\exists k>0$ such that $M^{\circ}=\Delta_{A \rightarrow B}^{k} M^{00}$. By corollary $5.7 \Delta_{A \rightarrow B}^{k} M^{00} \Longrightarrow+\lambda y$ : $A . \Delta_{B}^{k}\left(M^{\circ \circ}\left(\Delta_{A}^{k} y\right)\right)$ and the the sequence has no expansion rules.

The last term is equal to $\lambda y: A \cdot \Delta_{\mathcal{B}}^{k}\left(M^{\circ 0} y^{\circ}\right)=\lambda y: A \cdot(M y)^{\circ}=(\lambda y: A . M y)^{\circ}$ and then the property holds.

- If $M: \mathbf{T}$ and $M \stackrel{\text { Top }}{\longrightarrow} *$.

By lemma $5.5 M^{\circ}: \mathbf{T}$ and so $M^{\circ} \stackrel{\text { Top }}{\longrightarrow} *=*^{\circ}$.

Using 5.10 we can show now:

Theorem 5.13 (Simulation) If $\Gamma \vdash M: A$ and $M \Longrightarrow N$, then

1. $\exists k \geq 0$ such that $M^{\circ 0} \Longrightarrow+\Delta_{A}^{k} N^{\circ 0}$ if $M^{-\eta,-\delta} \stackrel{\longrightarrow}{\longrightarrow} N$

2. $M^{\circ} \Longrightarrow+N^{\circ}$

and there are no expansions in these reduction sequences. 
Proof. We show the property by induction on the structure of $M$. More precisely, for the first statement we analyze each case, while for the second there are two cases:

- if $M \stackrel{n .5}{\longrightarrow} N$, then apply lemma 5.12

- if $M_{\stackrel{-n}{\longrightarrow}}^{\longrightarrow} N$, then it is enough to analyze only the cases such that $M^{\circ}=M^{\circ 0}$, because when $M^{\circ}=\Delta_{A}^{h} M^{\circ 0}($ for $h>0)$ we have easily:

$$
\begin{aligned}
& M^{\circ}=\Delta_{A}^{h} M^{\circ 0} \Longrightarrow^{+} \text {by the first statement } \\
& \Delta_{A}^{h} \Delta_{A}^{k} N^{\circ 0}= \\
& \Delta_{A}^{h+k} N^{\circ 0}= \\
& N^{\circ}
\end{aligned}
$$

In order to conclude that the reductions in the statements of the lemma contain no expansions, it suffices to notice that every reduction built in the following proof contains no expansion steps: indeed it is constructed from one-step reductions that are not expansions or from reductions obtained by induction hypothesis (and thus without expansions) or from reductions obtained by lemma 5.12 , lemma 5.7, (again without expansions).

Now, we can analyze the cases involved in the proof of the first and the second statement.

- $M \equiv *$. It is in normal form.

- $M \equiv x$. The only possible case is $x \stackrel{\text { Top }}{\longrightarrow} *$, where $x: T$. Then, $x^{\circ 0}=x \stackrel{\text { Top }}{\longrightarrow} *=\Delta_{\mathrm{T}}^{0} *^{\circ 0}$.

- $M \equiv\left(P_{1} Q_{1}\right)$.

- If $\left(P_{1} Q_{1}\right): \mathbf{T}$ and $\left(P_{1} Q_{1}\right) \stackrel{\text { Top }}{\longrightarrow} *$, then $\left(P_{1} Q_{1}\right)^{\circ 0}: \mathbf{T}$ by lemma 5.5 and $\left(P_{1} Q_{1}\right)^{\circ 0} \stackrel{\text { rop }}{\longrightarrow} *=$ $\Delta_{\mathrm{T}}^{0} *^{\circ 0}$.

- If $(\lambda x: C . R) Q_{1} \stackrel{\beta}{\longrightarrow} R\left[Q_{1} / x\right]$.

$$
\begin{aligned}
& \left((\lambda x: C . R) Q_{1}\right)^{\circ 0}= \\
& \left((\lambda x: C \cdot R)^{\circ \circ} Q_{1}^{\circ}\right)= \\
& \left(\left(\lambda x: C \cdot R^{\circ}\right) Q_{1}^{\circ}\right) \stackrel{\rho}{=} \\
& R^{\circ}\left[Q_{1}^{\circ} / x\right]=\Rightarrow^{*} \text { by lemma } 5.10 \\
& R\left[Q_{1} / x\right]^{\circ}= \\
& \Delta_{A}^{k} R\left[Q_{1} / x\right]^{\circ \circ}
\end{aligned}
$$

- If $\left(P_{1} Q_{1}\right) \Longrightarrow\left(P_{2} Q_{1}\right)$, where $P_{1} \Longrightarrow P_{2}$.

Since $P_{1} \stackrel{\rightarrow}{\longrightarrow} P_{2}$ because $\left(P_{1} Q_{1}\right) \Longrightarrow\left(P_{2} Q_{1}\right)$, we have by induction hypothesis a reduction sequence $P_{1}^{\circ 0} \Longrightarrow+\Delta_{B \rightarrow A}^{h} P_{2}^{\circ 0}$ without expansions. Then

$$
\begin{gathered}
\left(P_{1} Q_{1}\right)^{\circ \circ}= \\
P_{1}^{\circ \circ} Q_{1}^{\circ} \Longrightarrow+\text { by induction hypothesis since } P_{1} \stackrel{-n=0}{\Longrightarrow} P_{2} \\
\left(\Delta_{B \rightarrow A}^{h} P_{2}^{\circ \circ}\right) Q_{1}^{\circ} \\
\text { If } h=0 \text {, then }\left(P_{2}^{\circ \circ} Q_{1}^{\circ}\right)=\left(P_{2} Q_{1}\right)^{\circ \circ} .
\end{gathered}
$$


If $h>0$, then

$\left(\Delta_{B \rightarrow A}^{h} P_{2}^{\circ \circ}\right) Q_{1}^{\circ}$

$\Downarrow_{+}$by lemma 5.7

$\left(\lambda w: B . \Delta_{A}^{h}\left(P_{2}^{\circ \circ}\left(\Delta_{B}^{h} w\right)\right)\right) Q_{1}^{\circ}$

$\downarrow \beta$

$\Delta_{A}^{h}\left(P_{2}^{\circ \circ}\left(\Delta_{B}^{h} Q_{1}^{\circ}\right)\right)$

$\Downarrow *$ by corollary 5.9

$\Delta_{A}^{h}\left(P_{2}^{\circ \circ} Q_{1}^{\circ}\right)$

$\bar{\Delta}_{A}^{h}\left(P_{2} Q_{1}\right)^{\circ 0}$

- If $\left(P_{1} Q_{1}\right) \Longrightarrow\left(P_{1} Q_{2}\right)$, where $Q_{1} \Longrightarrow Q_{2}$

$$
\begin{aligned}
& \left(P_{1} Q_{1}\right)^{\circ 0}= \\
& P_{1}^{\circ 0} Q_{1}^{\circ} \Longrightarrow+\text { by induction hypothesis } \\
& P_{1}^{\circ 0} Q_{2}^{\circ}= \\
& \left(P_{1} Q_{2}\right)^{\circ \circ}
\end{aligned}
$$

- $M \equiv\left\langle P_{1}, Q_{1}\right\rangle$

- If $\left\langle P_{1}, Q_{1}\right\rangle \Longrightarrow\left\langle P_{2}, Q_{1}\right\rangle$, where $P_{1} \Longrightarrow P_{2}$.

1 .

$$
\begin{aligned}
& \left\langle P_{1}, Q_{1}\right)^{\circ 0} \\
& \left\langle P_{1}^{\circ}, Q_{1}^{\circ}\right\rangle+\text { by induction hypothesis } \\
& \left\langle P_{2}^{\circ}, Q_{1}^{\circ}\right\rangle= \\
& \left\langle P_{2}, Q_{1}\right)^{\circ \circ}= \\
& \Delta_{A}^{0}\left(P_{2}, Q_{1}\right)^{\circ 0}
\end{aligned}
$$

2. Since $\langle P, Q\rangle^{\circ \circ}=\langle P, Q\rangle^{\circ}$, we have $\left\langle P_{1}, Q_{1}\right\rangle^{\circ} \Longrightarrow+\left\langle P_{2}, Q_{1}\right\rangle^{\circ}$ by the previous statement.

- If $\left\langle P_{1}, Q_{1}\right\rangle \Longrightarrow\left\langle P_{1}, Q_{2}\right\rangle$, where $Q_{1} \Longrightarrow Q_{2}$.

1.

$$
\begin{aligned}
& \left\langle P_{1}, Q_{1}\right\rangle^{\circ 0} \\
& \left\langle P_{1}^{\circ}, Q_{1}^{\circ}\right\rangle \Rightarrow+\text { by induction hypothesis } \\
& \left\langle P_{1}^{\circ}, Q_{2}^{\circ}\right\rangle= \\
& \left\langle P_{1}, Q_{2}\right\rangle^{\circ 0}= \\
& \Delta_{A}^{0}\left\langle P_{1}, Q_{2}\right)^{\circ 0}
\end{aligned}
$$

2. Since $\langle P, Q\rangle^{\circ 0}=\langle P, Q\rangle^{\circ}$, we have $\left(P_{1}, Q_{1}\right\rangle^{\circ} \Longrightarrow^{+}\left\langle P_{1}, Q_{2}\right\rangle^{\circ}$ by the previous statement.

- $M \equiv \lambda x: A . P_{1}$

Then $\lambda x: A . P_{1} \Longrightarrow \lambda x: A . P_{2}$, where $P_{1} \Longrightarrow P_{2}$.

1.

$$
\begin{aligned}
& \left(\lambda x: A \cdot P_{1}\right)^{\circ 0} \\
& \lambda x: A \cdot P_{1}^{\circ} \Longrightarrow+\text { by induction hypothesis } \\
& \lambda x: A \cdot P_{2}^{\circ}= \\
& \Delta_{A \rightarrow B}^{0}\left(\lambda x: A \cdot P_{2}\right)^{\circ 0}
\end{aligned}
$$


2. Since $\left(\lambda x: A . P_{i}\right)^{\circ}=\left(\lambda x: A . P_{i}\right)^{\circ 0}$ we have $\left(\lambda x: A . P_{1}\right)^{\circ} \Rightarrow+\left(\lambda x: A . P_{2}\right)^{\circ}$ by the previous statement.

- $M \equiv i n_{C}^{i}\left(P_{1}\right)$, for $i=1,2$ where $P_{1} \Longrightarrow P_{2}$.

Then $i n_{C}^{i}\left(P_{1}\right) \Longrightarrow i n_{C}^{i}\left(P_{2}\right)$

$$
\begin{aligned}
& i n_{C}^{i}\left(P_{1}\right)^{\circ 0}= \\
& i n_{C}^{i}\left(P_{1}^{\circ}\right) \Longrightarrow+\text { by induction hypothesis } \\
& i n_{C}^{i}\left(P_{2}^{\circ}\right)= \\
& \Delta_{B+C^{0}}^{0} n_{C}^{i}\left(P_{2}\right)^{\circ 0}
\end{aligned}
$$

- $M \equiv \pi_{i}\left(P_{1}\right)$, for $i=1,2$.

- If $\pi_{i}\left(P_{1}\right): \mathbf{T}$ and $\pi_{i}\left(P_{1}\right) \stackrel{\text { Top }}{\longrightarrow} *$, then $\pi_{i}\left(P_{1}\right)^{00}: \mathbf{T}$ by lemma 5.5 and $\pi_{i}\left(P_{1}\right)^{00} \stackrel{\text { Top }}{\longrightarrow} *=$ $\Delta_{\text {Top }}^{0} *^{\circ 0}$.

- If $\pi_{i}\left(P_{1}\right) \Longrightarrow \pi_{i}\left(P_{2}\right)$, where $P_{1} \Longrightarrow P_{2}$.

Since $P_{1} \stackrel{\neg \delta}{\longrightarrow} P_{2}$ because $\pi_{i}\left(P_{1}\right) \Longrightarrow \pi_{i}\left(P_{2}\right)$, we have by induction hypothesis a reduction sequence $P_{1}^{\circ \circ} \Longrightarrow+\Delta_{B \times A}^{h} P_{2}^{\circ \circ}$ without expansions. Then

$$
\begin{aligned}
& \pi_{i}\left(P_{1}\right)^{\circ 0}= \\
& \pi_{i}\left(P_{1}^{\circ 0}\right) \Longrightarrow+\text { by induction hypothesis } \\
& \pi_{i}\left(\Delta_{A_{1} \times A_{2}}^{h} P_{2}^{\circ 0}\right)
\end{aligned}
$$

If $h=0$, then $\pi_{i}\left(P_{2}^{\circ \circ}\right)=\pi_{i}\left(P_{2}\right)^{\circ}$.

If $h>0$, then

$$
\pi_{i}\left(\Delta_{A_{1} \times A_{2}}^{h} P_{2}^{\circ \circ}\right)
$$

$\Downarrow_{+}$by lemma 5.7

$$
\begin{aligned}
& \pi_{i}\left(\left\langle\Delta_{A_{1}}^{h} \pi_{1}\left(P_{2}^{\circ \circ}\right), \Delta_{A_{2}}^{h} \pi_{2}\left(Y_{2}^{\circ \circ}\right)\right\rangle\right) \\
& \Downarrow_{\pi_{i}}
\end{aligned}
$$

$$
\begin{aligned}
& \Delta_{A_{i}}^{h} \pi_{i}\left(P_{2}^{00}\right) \\
& \overline{\Delta_{A_{i}}^{h} \pi_{i}}\left(P_{2}\right)^{00}
\end{aligned}
$$

- $M \equiv \operatorname{Case}\left(P_{1}, R_{1}, N_{1}\right)$

- If $\operatorname{Case}\left(P_{1}, R_{1}, N_{1}\right): \mathbf{T}$ and $\operatorname{Case}\left(P_{1}, R_{1}, N_{1}\right) \stackrel{\text { Top }}{\longrightarrow}$, then $\operatorname{Case}\left(P_{1}, R_{1}, N_{1}\right)^{\circ 0}: \mathbf{T}$ by lemma 5.5 and $\pi_{i}\left(P_{1}\right)^{00} \stackrel{\text { Top }}{\longrightarrow} *=\Delta_{\text {Top }}^{0} *^{\circ 0}$.

$-\operatorname{Case}\left(i_{C_{1}+C_{2}}^{i}(S), R_{1}, R_{2}\right) \stackrel{\bullet}{\longrightarrow} R_{i} S$

$$
\begin{aligned}
& \text { Case }\left(i_{\mathrm{C}_{1}+C_{2}}^{i}(S), R_{1}, R_{2}\right)^{00}= \\
& \text { Case }\left(i_{C_{1}+C_{2}}^{i}(S)^{\circ}, R_{1}^{\circ}, R_{2}^{\circ}\right)= \\
& \operatorname{Case}\left(i n_{C_{1}+C_{2}}^{i_{1}}\left(S^{\circ}\right), R_{1}^{\circ}, R_{2}^{\circ}\right) \stackrel{\circ}{\Rightarrow} \\
& R_{1}^{\circ} S^{\circ}= \\
& \left(\Delta_{C_{i} \rightarrow A}^{h} R_{1}^{\circ o}\right) S^{\circ} \Longrightarrow+\text { by lemma } 5.7 \\
& \left(\lambda w^{i}: C_{i} \cdot \Delta_{A}^{h}\left(R_{1}^{\circ o}\left(\Delta_{C_{i}}^{h} w\right)\right)\right) S^{\circ} \stackrel{B}{\longrightarrow} \\
& \Delta_{A}^{h}\left(R_{1}^{\circ \circ}\left(\Delta_{C_{1}}^{h} S^{\circ}\right)\right) \Longrightarrow \text { by corollary } 5.9 \\
& \Delta_{A}^{h}\left(R_{1}^{\circ \circ} S^{\circ}\right)= \\
& \Delta_{A}^{h}\left(R_{1} S\right)^{\circ 0}
\end{aligned}
$$


- $\operatorname{Case}\left(P_{1}, R_{1}, N_{1}\right) \Longrightarrow \operatorname{Case}\left(P_{2}, R_{1}, N_{1}\right)$, where $P_{1} \Longrightarrow P_{2}$.

$\operatorname{Case}\left(P_{1}, R_{1}, N_{1}\right)^{\circ 0}=$

$\operatorname{Case}\left(P_{1}^{\circ}, R_{1}^{\circ}, N_{1}^{\circ}\right) \Longrightarrow+$ by induction hypothesis since

$\operatorname{Case}\left(P_{2}^{\circ}, R_{1}^{\circ}, N_{1}^{\circ}\right)=$

$\operatorname{Case}\left(P_{2}, R_{1}, N_{1}\right)^{\circ \circ}=$

$\Delta_{A}^{0} \operatorname{Case}\left(P_{2}, R_{1}, N_{1}\right)^{\circ 0}$

- $\operatorname{Case}\left(P_{1}, R_{1}, N_{1}\right) \Longrightarrow \operatorname{Case}\left(P_{1}, R_{2}, N_{1}\right)$, where $R_{1} \Longrightarrow R_{2}$

$\operatorname{Case}\left(P_{1}, R_{1}, N_{1}\right)^{\circ 0}=$

$\operatorname{Case}\left(P_{1}^{\circ}, R_{1}^{\circ}, N_{1}^{\circ}\right) \Longrightarrow+$ by induction hypothesis

$\operatorname{Case}\left(P_{1}^{\circ}, R_{2}^{\circ}, N_{1}^{\circ}\right)=$

$\operatorname{Case}\left(P_{1}, R_{2}, N_{1}\right)^{\circ \circ}=$

$\Delta_{A}^{0} \operatorname{Case}\left(P_{1}, R_{2}, N_{1}\right)^{\circ 0}$

- $\operatorname{Case}\left(P_{1}, R_{1}, N_{1}\right) \Longrightarrow \operatorname{Case}\left(P_{1}, R_{1}, N_{2}\right)$, where $N_{1} \Longrightarrow N_{2}$

$\operatorname{Case}\left(P_{1}, R_{1}, N_{1}\right)^{\circ 0}=$

$\operatorname{Case}\left(P_{1}^{\circ}, R_{1}^{\circ}, N_{1}^{\circ}\right) \Longrightarrow+$ by induction hypothesis

$\operatorname{Case}\left(P_{1}^{\circ}, R_{1}^{\circ}, N_{2}^{\circ}\right)=$

$\operatorname{Case}\left(P_{1}, R_{1}, N_{2}\right)^{\circ 0}=$

$\Delta_{A}^{0} \operatorname{Case}\left(P_{1}, R_{1}, N_{2}\right)^{00}$

- $M \equiv\left(\operatorname{rec} y: B \cdot P_{1}\right)^{i}$

- If $\left(\operatorname{rec} y: \mathbf{T} . P_{1}\right)^{i}: \mathbf{T}$ and $\left(\operatorname{rec} y: \mathbf{T} . P_{1}\right)^{i} \stackrel{\text { Top }}{\longrightarrow} *$, then $\left(\operatorname{rec} y: \mathbf{T} . P_{1}\right)^{i 00}: \mathbf{T}$ by lemma 5.5 and $\left(\text { rec } y: \text { T. } P_{1}\right)^{i 00} \stackrel{\text { Tor }}{\longrightarrow} *=\Delta_{\text {Top }}^{0} *^{\circ 0}$.

$-\left(\operatorname{rec} y: A . P_{1}\right)^{i} \stackrel{\text { rec }}{\longrightarrow} P_{1}\left[\left(\operatorname{rec} y: A . P_{1}\right)^{i-1} / y\right]$

$\left(\operatorname{rec} y: A . P_{1}\right)^{i 00}=$

$\left(\operatorname{rec} y: A . P_{1}^{\circ}\right)^{i} \stackrel{\text { rac }}{\longrightarrow}$

$P_{1}^{\circ}\left[\left(\operatorname{rec} y: A . P_{1}^{0}\right)^{i-1} / y\right]=$

$P_{1}^{\circ}\left[\left(\text { rec } y: A . P_{1}\right)^{i-100} / y\right] \Longrightarrow^{*}$ by lemma 5.8

$\left(P_{1}\left[\left(\operatorname{rec} y: A \cdot P_{1}\right)^{i-1} / y\right]\right)^{\circ}=$

$\Delta_{A}^{h}\left(P_{1}\left[\left(\operatorname{rec} y: A . P_{1}\right)^{i-1} / y\right]\right)^{\circ 0}$

$-\left(\operatorname{rec} y: A \cdot P_{1}\right)^{i} \Longrightarrow\left(\operatorname{rec} y: A \cdot P_{2}\right)^{i}$.

$\left(\operatorname{rec} y: A . P_{1}\right)^{i 00}=$

$\left(\text { rec } y: A . P_{1}^{\circ}\right)^{i} \Longrightarrow+$ by induction hypothesis

$\left(\operatorname{rec} y: A \cdot P_{2}^{0}\right)^{i}=$

$\Delta_{A}^{0}\left(\operatorname{rec} y: A . P_{2}\right)^{i 00}$

\subsection{Strong Normalization of the Full Calculus}

Having shown that our translation satisfies the hypothesis of Proponition 5.1, all wc are now left to prove is that the bounded reduction system without expansion rules is strongly normalizing. This can be established by one of the standard techniques of reducibility, and does not present essential difficulties once the right definitions of stability or reducibility are given. In 6 and 7 we provide two proofs, one adapting the proof provided by Poigne and Voss in [PV87], and the other adapting Girard's proof from [GLT90]. It is then finally possible to state the following

\section{Theorem 5.14 (Strong normalization)}

The reduction $\Longrightarrow$ for the bounded system with expansions is strongly normalizing.

Proof. By proposition 5.1, theorem 5.13 and Corollary 6.11 (or 7.7). 


\section{Strong Normalization via stability}

We have shown that every reduction sequence starting at $M$ involving the rules presented in section 3 can be simulated by the translation of $M$ in the same calculus but without expansions. We will show in this section that the calculus without expansions is strongly normalizing.

\subsection{Stability}

We define a set of stable terms of type $A$ by induction on the type $A$ in the following way:

- For $M$ of atomic type $A, M$ is stable if and only if it is strongly normalizing.

- For $M$ of type $A_{1} \times A_{2}, M$ is stable if and only if it is strongly normalizing and whenever $M$ reduces to $\left\langle M_{1}, M_{2}\right\rangle$, then $M_{1}$ and $M_{2}$ are stable terms of type $A_{1}$ and $A_{2}$ respectively.

- For $M$ of type $A_{1}+A_{2}, M$ is stable if and only if it is strongly normalizing and whenever $M$ reduces to $i n_{A_{1}+A_{2}}^{i}\left(M^{\prime}\right)$ then $M^{\prime}$ is stable of type $A_{i}$.

- For $M$ of type $A_{1} \rightarrow A_{2}, M$ is stable if and only if for every stable term $N$ of type $A_{1}, M N$ is a stable term of type $A_{2}$.

Proposition 6.1 Let $M$ be a term of type $A \rightarrow B$

1. if $M$ is stable, then $M Q_{1} \ldots Q_{k}$ is stable for arbitrary stablc $Q_{1} \ldots Q_{k}$ of appropriate types,

2. if $M Q_{1} \ldots Q_{k}$ is stable for arbitrary stable $Q_{1} \ldots Q_{k}$ of appropriate types, then $M$ is stable.

Proof.

By induction on $k$. For $k=1$, this is just our definition of stability, while for $k>1$ :

1. we know by induction hypothesis that $M Q_{1} \ldots Q_{k-1}$ is stable, and then $M Q_{1} \ldots Q_{k}$ is stable by definition since $Q_{k}$ is stable too.

2. if $M Q_{1} \ldots Q_{k}$ is stable for arbitrary stable terms $Q_{1} \ldots Q_{k}$, then by definition of stability, $M Q_{1} \ldots Q_{k-1}$ is stable for arbitrary $Q_{1} \ldots Q_{k-1}$, and then by induction hypothesis $M$ is stable.

Remark 6.2 We can then use equivalently as a definition for stability $k>1$ or $k=1$. We will use in the following the most suitable one for each case.

Notation 6.3 In what follows we will use often $\vec{Q}$ to denote a sequence of terms $Q_{1} \ldots Q_{k}$, with the convention that $M \vec{Q}$ really stands for $M Q_{1} \ldots Q_{k}$.

Lemma 6.4

- If $M, N, \vec{Q}$ are strongly normalizing, then the terms $x \vec{Q}, M x, i_{A}^{i}(M),(\text { rec } y: B \cdot M)^{0} \vec{Q}$ and $\langle M, N\rangle$ are strongly normalizing.

- If $M, \vec{Q}$ are strongly normalizing and $M$ has product type but does not reduce to a pair, the $\pi_{i}(M) \vec{Q}$ is strongly normalizing.

- If $P, M, N, \vec{Q}$ are strongly normalizing and $P$ has sum type but does not reduce to $i^{i}$, then Case $(P, M, N) \vec{Q}$ is strongly normalizing.

Proof. The argument of the proof is the same for all the cases: we show that a generic reduction sequence starting from the given term always terminates. We assume in what follows that no Top rule is applied at the root of our term during the reduction, as in this case the reduction sequence simply stops and is then clearly finite. 
- $-x \vec{Q}$. There are no expansions in this system, so a reduction starting at $x \vec{Q}$ can only proceed in the $Q_{i}^{\prime} s$. Since the $\vec{Q}$ are strongly normalizing, this means that the reduction sequence also terminates, so we conclude that $x \vec{Q}$ is strongly normalizing.

- $M x$. Consider a given reduction sequence starting from $M x$. There are two possibilities:

* $M$ does not reduce to a $\lambda$-expression in the reduction sequence. Then, the reduction sequence starting at $M x$ terminates because it is of the form $M x \Longrightarrow M_{1} x \Longrightarrow$ $\ldots \Rightarrow M_{n} x$, where $M \Rightarrow M_{1} \Rightarrow \ldots \Rightarrow M_{n}$ is a terminating reduction sequence starting at $M$.

* $M$ reduces at some point to $\lambda y: B . P$. Then the reduction sequence starting at $M x$ is of the form $M x \Longrightarrow^{*}(\lambda y: B . P) x^{\prime} \Longrightarrow^{*}\left(\lambda y: B . P^{\prime}\right) x^{\prime \prime} \Longrightarrow P^{\prime}\left[x^{\prime \prime} / y\right] \Longrightarrow \ldots$, where $P \Longrightarrow^{*} P^{\prime}$ and $x \Longrightarrow^{*} x^{\prime} \Longrightarrow^{*} x^{\prime \prime}$ (because $x$ can reduce to $*$ ). On the other hand $P^{\prime}$ is strongly normalizing as $P$ is, and thus also $P^{\prime}[x / y]$ and $P^{\prime}[* / y]$ must be strongly normalizing ${ }^{6}$. Therefore the reduction sequence is terminating.

We conclude that $M x$ is strongly normalizing.

- $i n_{A_{1}+A_{3}}^{i}(M)$. A reduction sequence starting at $i n_{A_{1}+A_{2}}^{i}(M)$ is of the form $i n_{A_{1}+A_{2}}^{i}(M)$ $\Longrightarrow i_{A_{1}+A_{2}}^{i}\left(M_{1}\right) \Longrightarrow i n_{A_{1}+A_{2}}^{i}\left(M_{2}\right) \Longrightarrow \ldots$, where $M \Longrightarrow M_{1} \Longrightarrow M_{2} \Longrightarrow \ldots$ since the leftmost $i n^{i}$ cannot be removed. Since $M$ is strongly normalizing, these two sequences are both finite.

- $(\text { rec } y: B . M)^{0} \vec{Q}$. Since the term $(\text { rec } y: B . M)^{0}$ cannot be reduced at the root position (neither by a rec-rule nor by a Top-rule), then only internal reductions in $M$ are possible. Therefore a reduction starting at (rec $y: B . M)^{0} \vec{Q}$ only proceeds in $M, \vec{Q}$ that are strongly normalizing hy hypothesis. Hence, the reduction sequence is finite.

$-\langle M, N\rangle$. A reduction sequence starting at $\langle M, N\rangle$ is of the form $\langle M, N\rangle \Rightarrow^{*}\left\langle M_{1}, N_{1}\right\rangle$ $\Longrightarrow^{*}\left\langle M_{2}, N_{2}\right\rangle \Longrightarrow^{*} \ldots$, where $M \Longrightarrow^{*} M_{1} \Longrightarrow^{*} M_{2} \Longrightarrow^{*} \ldots$ and $N \Longrightarrow{ }^{*} N_{1}$ $\Longrightarrow^{*} N_{2} \Longrightarrow{ }^{*}$... Since $M$ and $N$ are strongly normalizing, these reduction sequences are all finite.

- $\pi_{i}(M) \vec{Q}$. Since by hypothesis $M$ does not reduce to a pair, the leftmost $\pi_{i}$ cannot be removed and therefore a reduction starting at $\pi_{\boldsymbol{i}}(M) \vec{Q}$ only proceeds in $M, \vec{Q}$ that are strongly normalizing by hypothesis. Therefore the reduction sequence is finite.

- $\operatorname{Case}(P, M, N) \vec{Q}$. Since by hypothesis $P$ does not reduce to an $i n^{i}$, the leftmost $C a s e$ cannot be removed and therefore a reduction starting at $\operatorname{Case}(P, M, N) \vec{Q}$ only proceeds in $P, M, N, \vec{Q}$ that are strongly normalizing by hypothesis. Hence, the reduction sequence terminates.

\subsection{Properties of stability}

\section{Lemma 6.5}

1. Every stable term $M$ of type $A$ is strongly normalizing.

2. A term $x \vec{Q}$ of type $A$ is stable for arbitrary strongly normalizing terms $\vec{Q}$.

Proof. By induction on the type $A$

- If $A$ is not a functional type:

1. By definition.

\footnotetext{
${ }^{6}$ From any infinite sequence starting from $P^{\prime}[x / y]$ or $P^{\prime}[* / y]$ we can build an infinite sequence starting from $P^{\prime}$.
} 
2. By lemma $6.4 x \vec{Q}$ is strongly normalizing. As pointed out in the proof of lemma 6.4, either $x \vec{Q}$ reduces to *, or reductions from $x \vec{Q}$ can only proceed in the $Q_{i}^{\prime} s$. Therefore, $x \vec{Q}$ cannot reduce to a pair, nor to an $i n^{i}$ and thus $x \vec{Q}$ is stable by definition.

- If $A$ is a functional type:

1. Let $M$ be a stable term of type $A \equiv B \rightarrow C$ and let $x$ be a variable of type $B$. By the second induction hypothesis (with $n=0$ ) $x: B$ is stable and so by definition $M x$ is also stable. By lemma $6.4 M x$ is strongly normalizing. Suppose now that $M$ is not strongly normalizing. Then there is an infinite reduction sequence $M \Longrightarrow M_{1} \Rightarrow \ldots$ and thus an infinite reduction sequence $M x \Longrightarrow M_{1} x \Rightarrow \ldots$ which leads to a contradiction. Therefore $M$ is strongly normalizing.

2. Let $x \vec{Q}$ be of type $B \rightarrow C$ with all the $Q_{i}^{\prime} s$ strongly normalizing. Let $N$ be any stable term of type $B$. From the first induction hypothesis $N$ is also strongly normalizing and applying the second induction hypothesis $x \vec{Q} N$ is stable of type $C$, so by proposition 6.1 $x \vec{Q}$ is stable.

Lemma 6.6 If $M$ is a stable term of type $A$ and $M \Longrightarrow N$, then $N$ is stable.

Proof. We first recall that $N$ is also of type $A$ by proposition 3.7 . We show the property by induction on $A$.

- $A$ is not a functional type: $M$ is strongly normalizing and then also $N$ is strongly normalizing since for every reduction sequence $N \Longrightarrow{ }^{\prime}$ there is a longer reduction sequence $M \Longrightarrow N$ $\Longrightarrow^{*} N^{\prime}$ that terminates. On the other hand, when $N \Longrightarrow^{*}\left\langle M_{1}, M_{2}\right\rangle$, then $M_{1}$ and $M_{2}$ are stable, because $M \Longrightarrow^{*} N \Longrightarrow \Longrightarrow^{*}\left\langle M_{1}, M_{2}\right\rangle$ and $M$ is stable. Similarly, if $N \Longrightarrow^{*}$ in ${ }_{B}^{i}\left(M^{\prime}\right)$ we have $M \Longrightarrow{ }^{*} N \Longrightarrow i n_{B}^{i}\left(M^{\prime}\right)$ and then $M^{\prime}$ is stable. Therefore $N$ is stable.

- $A$ is a functional type: by definition of stability on arrow types, it suffices to show that $N M_{1}$ is stable for any stable term $M_{1}$. Now, given a stable $M_{1}, M M_{1}$ is stable because $M$ is, and $M M_{1} \Longrightarrow N M_{1}$, so by induction hypothesis $N M_{1}$ is stable.

\subsection{Products, sums and basic recursion}

\section{Lemma 6.7}

- $\pi_{1}(M), \pi_{2}(M)$ and $i_{A_{2}+A_{2}}(M)$ are stable if $M$ is stable.

- $\operatorname{Case}\left(P, M_{1}, M_{2}\right)$ is stable if $P, M_{1}$ and $M_{2}$ are stable.

- $\langle M, N\rangle$ is stable if $M$ and $N$ are stable.

- $(\text { rec } y: B . M)^{0}$ is stable if $M$ is stable.

Proof.

- We will prove that $\pi_{i}(M)$ is stable if $M$ is stable, for $i=1,2$. Assume that $A$ is the type of $\pi_{i}(M)$. Take stable terms $\vec{Q}$ such that $\pi_{i}(M) \vec{Q}$ is not of functional type. There are two cases to consider: 
- $M$ docs not reduce to a pair (recall that $M$ has a product type). By lemma $6.4 \pi_{i}(M) \vec{Q}$ is strongly normalizing. On the other hand, $\pi_{i}(M) \vec{Q}$ cannot reduce to a pair, nor to an $i n^{i}$ because either it reduces to $*$, or the $\pi_{i}$ cannot be removed. Therefore $\pi_{i}(M) \vec{Q}$ is stable for arbitrary stable terms $\vec{Q}$, so by proposition $6.1 \pi_{i}(M)$ is also stable.

$-M \Longrightarrow *\left\langle M_{1}, M_{2}\right\rangle$. Suppose $\pi_{i}(M) \vec{Q}$ has an infinite reduction sequence: such reduction has to remove $\pi_{i}$ in some step in the following way:

$$
\pi_{i}(M) \vec{Q} \Longrightarrow^{*} \pi_{i}\left(\left\langle M_{1}, M_{2}\right\rangle\right) \overrightarrow{Q^{\prime}} \Longrightarrow * M_{i}^{\prime} \overrightarrow{Q^{\prime \prime}} \Longrightarrow \ldots
$$

where $M_{i} \Longrightarrow^{*} M_{i}^{\prime}$ and $Q_{i} \Longrightarrow^{*} Q_{i}^{\prime} \Longrightarrow^{*} Q_{i}^{\prime \prime}$.

Since $M_{i}$ and $Q_{i}$ are stable, by lemma 6.6 also $M_{i}^{\prime}$ and $Q_{i}^{\prime \prime}$ are stable. Therefore $M_{i}^{\prime} \overrightarrow{Q^{\prime \prime}}$ is stable and then strongly normalizing. From the form of the reduction sequence we can conclude that $\pi_{i}(M) \vec{Q}$ is also strongly normalizing which leads to a contradiction. Therefore $\pi_{i}(M) \vec{Q}$ has no infinite reduction sequences and is strongly normalizing. Now, if $\pi_{i}(M) \vec{Q}$ reduces to a pair, the reduction removes the $\pi_{i}$ and we have necessarily

$$
\pi_{i}(M) \vec{Q} \Longrightarrow \pi_{i}\left(\left\langle M_{1}, M_{2}\right\rangle\right) \vec{Q}^{\prime} \Longrightarrow^{*} M_{i}^{\prime} \overrightarrow{Q^{\prime \prime}} \Longrightarrow *\left\langle L_{1}, L_{2}\right\rangle
$$

Since $M_{i}^{\prime} \overrightarrow{Q^{\prime \prime}}$ is stable, $L_{1}$ and $L_{2}$ are stable.

If $\pi_{i}(M) \vec{Q}$ reduces to $i n_{C}^{i}(L)$, the reduction removes the $\pi_{i}$ and we have necessarily

$$
\pi_{i}(M) \vec{Q} \Longrightarrow^{*} \pi_{i}\left(\left\langle M_{1}, M_{2}\right\rangle\right) \overrightarrow{Q^{\prime}} \Longrightarrow M_{i}^{\prime} \overrightarrow{Q^{\prime \prime}} \Longrightarrow^{*} i n_{C}^{i}(L)
$$

Since $M_{i}^{\prime} \overrightarrow{Q^{\prime \prime}}$ is stable, $L$ is also stable.

We can conclude that $\pi_{i}(M) \vec{Q}$ is stable and by proposition $6.1 \pi_{i}(M)$ is stable.

- We will prove that $i n_{A_{1}+A_{2}}^{i}(M)$ is stable if $M$ is stable. Since $M$ is stable, $M$ is strongly normalizing and by lemma 6.4 also $i n_{A_{1}+A_{2}}^{i}(M)$ is strongly normalizing. On the other hand $i n_{A_{1}+A_{2}}^{i}(M)$ cannot reduce to a pair because it is of sum type. If $i n_{A_{1}+A_{2}}^{i}(M) \Rightarrow^{*} i n_{A_{1}+A_{2}}^{i}\left(M^{\prime}\right)$, then $M \Longrightarrow{ }^{*} M^{\prime}$ and since $M$ is stable by hypothesis, we have that $M^{\prime}$ is stable by lemma 6.6. We conclude that $\operatorname{in}_{A_{1}+A_{2}}^{i}(M)$ is stable.

- We will prove that $\operatorname{Case}\left(P, M_{1}, M_{2}\right)$ is stable if $P, M_{1}$ and $M_{2}$ are stable. Assume that $A$ is the type of $\operatorname{Case}\left(P, M_{1}, M_{2}\right)$. Take stable terms $\vec{Q}$ such that $\operatorname{Case}\left(P, M_{1}, M_{2}\right) \vec{Q}$ is not of functional type. Since $P$ is of sum type it cannot reduce to a pair, but it could reduce to an $i n_{B}^{i}\left(P^{\prime}\right)$. There are two cases to consider:

- $P$ cannot reduce to an $i n^{i}$. Then by lemma $6.4 \operatorname{Case}\left(P, M_{1}, M_{2}\right) \vec{Q}$ is also strongly normalizing. On the other hand, $\operatorname{Case}\left(P, M_{1}, M_{2}\right) \vec{Q}$ cannot reduce to a pair, nor to an $i n^{i}$ because either $\operatorname{Case}\left(P, M_{1}, M_{2}\right) \vec{Q}$ reduces to $*$, or the Case construction cannot be removed. Therefore $\operatorname{Case}\left(P, M_{1}, M_{2}\right) \vec{Q}$ is stable and by proposition $6.1 \operatorname{Case}\left(P, M_{1}, M_{2}\right)$ is stable.

- $P$ can reduce to some $i n_{B}^{i}\left(P^{\prime}\right)$. Consider a reduction starting from $\operatorname{Case}\left(P, M_{1}, M_{2}\right) \vec{Q}$ : if in this reduction the $\operatorname{Case}\left(P, M_{1}, M_{2}\right)$ is never eliminated, then the reduction sequence actually proceeds inside the $P, M_{1}, M_{2}, \vec{Q}$, that are all strongly normalizing as they are 
stable, so this reduction sequence is finite. Otherwise, this reduction sequence removes the Case constructor and looks like:

$$
\operatorname{Case}\left(P, M_{1}, M_{2}\right) \vec{Q} \Longrightarrow{ }^{*} \operatorname{Case}\left(i n_{B}^{i}\left(P^{\prime}\right), M_{1}^{\prime}, M_{2}^{\prime}\right) \overrightarrow{Q^{\prime}} \Longrightarrow M_{i}^{\prime} P^{\prime} \overrightarrow{Q^{\prime}} \Longrightarrow \ldots
$$

where $M_{i} \Longrightarrow{ }^{*} M_{i}^{\prime}$ and $Q_{i} \Longrightarrow{ }^{*} Q_{i}^{\prime}$.

Since $P, M_{i}$ and the $Q_{i}$ in $\vec{Q}$ are stable, then $P^{\prime}$ is stable by definition and by lemma 6.6 also the $M_{i}^{\prime}$ and the $Q_{i}^{\prime}$ in $\overrightarrow{Q^{\prime}}$ are stable. Therefore $M_{i}^{\prime} P^{\prime} \overrightarrow{Q^{\prime}}$ is stable and then strongly normalizing, so the reduction $M_{i}^{\prime} P^{\prime} \overrightarrow{Q^{\prime}} \Longrightarrow \ldots$ is finite and then in turn the whole reduction sequence is finite. We conclude that $\operatorname{Case}\left(P, M_{1}, M_{2}\right) \vec{Q}$ is strongly normalizing. On the other hand, if $\operatorname{Case}\left(P, M_{1}, M_{2}\right) \vec{Q}$ reduces to a pair we have necessarily a reduction sequence removing the Case that looks like:

$$
\operatorname{Case}\left(P, M_{1}, M_{2}\right) \vec{Q} \Longrightarrow{ }^{*} \operatorname{Case}\left(i n_{B}^{i}\left(P^{\prime}\right), M_{1}^{\prime}, M_{2}^{\prime}\right) \overrightarrow{Q^{\prime}} \Longrightarrow M_{i}^{\prime} P^{\prime} \vec{Q}^{\prime} \Rightarrow^{*}\left\langle L_{1}, L_{2}\right\rangle
$$

As seen above, $M_{i}^{\prime} P^{\prime} \overrightarrow{Q^{\prime}}$ is stable, so $L_{1}$ and $L_{2}$ are stable too.

If $\operatorname{Case}\left(P, M_{1}, M_{2}\right) \vec{Q}$ reduces to $i_{C}^{i}(L)$, we have necessarily a reduction sequence removing the Case that looks like:

$$
\operatorname{Case}\left(P, M_{1}, M_{2}\right) \vec{Q} \Longrightarrow \text { Case }\left(i n_{B}^{i}\left(P^{\prime}\right), M_{1}^{\prime}, M_{2}^{\prime}\right) \overrightarrow{Q^{\prime}} \Longrightarrow M_{i}^{\prime} P^{\prime} \vec{Q}^{\prime} \Longrightarrow{ }^{*} n_{C}^{i}(L)
$$

Again, $M_{i}^{\prime} P^{\prime} \overrightarrow{Q^{\prime}}$ is stable, so $L$ is also stable.

We conclude that $\operatorname{Case}\left(P, M_{1}, M_{2}\right) \vec{Q}$ is stable and by proposition $6.1 \operatorname{Case}\left(P, M_{1}, M_{2}\right)$ is stable.

- We will prove that $\langle M, N\rangle$ is stable if $M$ and $N$ are stable. By hypothesis $M$ and $N$ are strongly normalizing and by lemma $6.4\langle M, N\rangle$ is strongly normalizing. It is clear that $\langle M, N\rangle$ cannot reduce to an $i^{i}$. If $\langle M, N\rangle \Longrightarrow^{*}\left\langle M^{\prime}, N^{\prime}\right\rangle$, then $M \Longrightarrow^{*} M^{\prime}$ and $N \Longrightarrow{ }^{*} N^{\prime}$. By hypothesis $M$ and $N$ are stable and then by lemma $6.6 M^{\prime}$ and $N^{\prime}$ are stable. We can conclude that $\langle M, N\rangle$ is stable.

- We will prove that (rec $y: B . M)^{0}$ is stable if $M$ is stable. Take arbitrary stable terms $\vec{Q}$ such that $(\text { rec } y: B . M)^{0} \vec{Q}$ is not of functional type. By lemma $6.4(\text { rec } y: B . M)^{\circ} \vec{Q}$ is strongly normalizing as the stable terms $M, \vec{Q}$ are strongly normalizing.

On the other hand, the term (rec $y: B \cdot M)^{0} \vec{Q}$ cannot reduce to a pair, nor to an $i n^{i}$ because either it reduces to $*$, or the rec with exponent 0 is not removed. Therefore $(\operatorname{rec} y: B . M)^{0} \vec{Q}$ is stable and by proposition $6.1(\operatorname{rec} y: B . M)^{0}$ is stable.

\subsection{Abstraction and recursion}

Lemma 6.8 If $M[N / x]$ is stable, then $(\lambda x: A . M) N$ is stable provided that $N$ is stable if $x$ is not free in $M$.

Proof. Let $\vec{Q}$ be stable terms such that $M[N / x] \vec{Q}$ is not of functional type. By proposition 6.1 $M[N / x] \vec{Q}$ is stable and then strongly normalizing. We want to show that $(\lambda x: A . M) N$ is stable, but this follows from proposition 6.1 once we show that $(\lambda x: A . M) N \vec{Q}$ is stable. 
Notice first of all that $N$ is strongly normalizing in any case: if $x$ is not free in $M$ we know by hypothesis that $N$ is stable, hence also strongly normalizing, while if $x$ is free in $M$, then $N$ is strongly normalizing because it is a subterm of the stable (hence strongly normalizing) term $M[N / x]$. Secondly, $M$ is also strongly normalizing, because from a non terminating reduction sequence starting at $M$ we can build a non terminating reduction sequence starting at $M[N / x]$, which is impossible, because this last term is stable, hence strongly normalizing.

We can now show that $(\lambda x: A . M) N \vec{Q}$ is strongly normalizing.

Every reduction sequence starting at $(\lambda x: A . M) N \vec{Q}$ yields to $*$, proceeds inside $M, N, \vec{Q}$ or looks like:

$$
(\lambda x: A . M) N \vec{Q} \Longrightarrow{ }^{*}\left(\lambda x: A \cdot M^{\prime}\right) N^{\prime} \vec{Q}^{\prime} \Longrightarrow M^{\prime}\left[N^{\prime} / x\right] \overrightarrow{Q^{\prime}} \Longrightarrow \ldots
$$

where $M \Rightarrow M^{\prime}, N \Longrightarrow N^{\prime}$ and $Q_{i} \Longrightarrow Q_{i}^{\prime}$.

In the first case the reduction stops at $*$, and in the second case the reduction is finite because the terms $M, N, \vec{Q}$ are all strongly normalizing $(\vec{Q}$ are stable, while $M$ and $N$ are strongly normalizing as seen above).

In the last case, by lemma 4.5 and corollary $4.8 M[N / x] \Longrightarrow^{*} M^{\prime}\left[N^{\prime} / x\right]$ and since $M[N / x]$ is stable by hypothesis, applying lemma 6.6 we obtain that $M^{\prime}\left[N^{\prime} / x\right]$ is also stable. Once again by lemma 6.6 every $Q_{i}^{\prime}$ is stable and by proposition $6.1 M^{\prime}\left[N^{\prime} / x\right] \overrightarrow{Q^{\prime}}$ is stable and hence strongly normalizing. Then $(\lambda x: A . M) N \vec{Q}$ is also strongly normalizing.

Hence we have proved that $(\lambda x: A . M) N \vec{Q}$ is strongly normalizing.

On the other hand, if $(\lambda x: A . M) N \vec{Q}$ reduces to a pair $\left\langle L_{1}, L_{2}\right\rangle$, we have necessarily to remove the redex $(\lambda x: A . M) N$ and we obtain a reduction sequence similar to the previous one with more steps from $M^{\prime}\left[N^{\prime} / x\right] \overrightarrow{Q^{\prime}}$ to a pair $\left\langle L_{1}, L_{2}\right\rangle$. Since $M^{\prime}\left[N^{\prime} / x\right] \overrightarrow{Q^{\prime}}$ is stable, $L_{1}$ and $L_{2}$ are stable. Similarly, if $(\lambda x: A . M) N \vec{Q}$ reduces to $i n_{B}^{i}(L)$, we obtain a reduction sequence with more steps from $M^{\prime}\left[N^{\prime} / x\right] \overrightarrow{Q^{\prime}}$ to a $i_{B}^{i}(L)$. Since $M^{\prime}\left[N^{\prime} / x\right] \overrightarrow{Q^{\prime}}$ is stable, $L$ is stable.

We can conclude that $(\lambda x: A . M) N \vec{Q}$ is stable and thus we have $(\lambda x: A . M) N$ stable by proposition 6.1 .

Lemma 6.9 If $M\left[(\operatorname{rec} y \cdot M)^{i-1} / y\right]$ is stable, then $(\operatorname{rec} y \cdot M)^{i}$ is stable.

Proof. Take stable terms $\vec{Q}$ such that (rec $y: B . M)^{i} \vec{Q}$ is not of functional type. We first show that (rec $y: B . M)^{i} \vec{Q}$ is strongly normalizing. For this, notice that any reduction starting from $(\operatorname{rec} y: B . M)^{\prime} \vec{Q}$ that does not eliminate the rec construct is finite, as it must proceed in the $M, \vec{Q}$, which are all stable and hence also strongly normalizing. We are left with those reductions that do eliminate the rec construct. Any such reduction sequence looks like:

$$
(\operatorname{rec} y: B . M)^{i} \vec{Q} \Longrightarrow *\left(\operatorname{rec} y: B \cdot M^{\prime}\right)^{i} \overrightarrow{Q^{\prime}} \Longrightarrow M^{\prime}\left[\left(\operatorname{rec} y: B \cdot M^{\prime}\right)^{i-1} / y\right] \overrightarrow{Q^{\prime}}
$$

where $Q_{i} \Longrightarrow^{*} Q_{i}^{\prime}, M \Longrightarrow^{*} M^{\prime}$ and hence also $M\left[(\text { rec } y: B \cdot M)^{i-1} / y\right] \Longrightarrow^{*} M^{\prime}\left[\left(\text { rec } y: B \cdot M^{\prime}\right)^{i-1} / y\right]$. Since $M\left[(\operatorname{rec} y: B . M)^{i-1} / y\right]$ and the $Q_{i}$ are stable, by lemma $6.4 M^{\prime}\left[\left(\operatorname{rec} y: B . M^{\prime}\right)^{i-1} / y\right]$ and the $Q_{i}^{\prime}$ are also stable, so $M^{\prime}\left[\left(\text { rec } y: B \cdot M^{\prime}\right)^{i-1} / y\right] \overrightarrow{Q^{\prime}}$ is stable and thus strongly normalizing. This means that the whole reduction sequence is finite, and concludes this first part of the proof.

Now, if (rec $y: B . M)^{i} \vec{Q}$ reduces to a pair, the rec has been removed and the reduction sequence looks like:

$$
(\operatorname{rec} y: B . M)^{i} \vec{Q} \Longrightarrow^{*}\left(\operatorname{rec} y: B \cdot M^{\prime}\right)^{i} \overrightarrow{Q^{\prime}} \Longrightarrow M^{\prime}\left[\left(\operatorname{rec} y: B \cdot M^{\prime}\right)^{i-1} / y\right] \overrightarrow{Q^{\prime}} \Longrightarrow *\left\langle L_{1}, L_{2}\right\rangle
$$

As $M^{\prime}\left[\left(\operatorname{rec} y: B \cdot M^{\prime}\right)^{i-1} / y\right] \overrightarrow{Q^{\prime}}$ is stable, $L_{1}$ and $L_{2}$ are also stable. 
Similarly, if (rec $y: B \cdot M)^{i} \vec{Q}$ reduces to $i n_{B}^{i}(L)$, the rec has been removed and the reduction sequence looks like:

$$
(\operatorname{rec} y: B \cdot M)^{i} \vec{Q} \Longrightarrow^{*}\left(\operatorname{rec} y: B \cdot M^{\prime}\right)^{i} \overrightarrow{Q^{\prime}} \Longrightarrow M^{\prime}\left[\left(\operatorname{rec} y: B \cdot M^{\prime}\right)^{i-1} / y\right] \overrightarrow{Q^{\prime}} \Longrightarrow^{*} i n_{B}^{i}(L)
$$

As $M^{\prime}\left[\left(\operatorname{rec} y: B . M^{\prime}\right)^{i-1} / y\right] \overrightarrow{Q^{\prime}}$ is stable, $L$ is also stable.

We can conclude that (rec $y: B . M)^{i} \vec{Q}$ is stable and thus by proposition $6.1(\operatorname{rec} y: B . M)^{i}$ is stable.

Lemma 6.10 Let $M$ be a term such that all the free variables are among $\left\{x_{i}\right\}_{i=1 \ldots n}$. If $\bar{N}$ are stable terms, then $M\left[N_{1} \ldots N_{n} / x_{1} \ldots x_{n}\right]$ is stable.

Proof. We show the property by induction on the structure of $M$.

- $M \equiv *$. Then $*\left[N / x_{i}\right]=*$ and $*$ is strongly normalizing and hence stable.

- $M \equiv x_{i}$. Then $x_{i}\left[N / x_{i}\right]=N$ and $N$ is stable by hypothesis.

- $M \equiv \operatorname{Case}(P, Q, R)$. We have $\operatorname{Case}(P, Q, R)[\bar{N} / \bar{x}]=\operatorname{Case}(P[\bar{N} / \bar{x}], Q[\bar{N} / \bar{x}], R[\bar{N} / \bar{x}]) . \quad$ By induction hypothesis the terms $P[\bar{N} / \bar{x}], Q[\bar{N} / \bar{x}]$ and $R[\bar{N} / \bar{x}]$ are stable, so we can apply lemma 6.7 and we get that $\operatorname{Case}(P[\bar{N} / \bar{x}], Q[\bar{N} / \bar{x}], R[\bar{N} / \bar{x}])$ is stable.

- $M \equiv \pi_{i}(P)$, for $i=1,2$. We have $\pi_{i}(P)[\bar{N} / \bar{x}]=\pi_{i}(P[\bar{N} / \bar{x}])$. By induction hypothesis $P[\bar{N} / \bar{x}]$ is stable and by lemma $6.7 \pi_{i}(P[\bar{N} / \bar{x}])$ is stable.

- $M \equiv i n_{B}^{i}(P)$, for $i=1,2$. We have $i n_{B}^{i}(P)[\bar{N} / \bar{x}]=i n_{B}^{i}(P[\bar{N} / \bar{x}])$. By induction hypothesis $P[\bar{N} / \bar{x}]$ is stable and by lemma $6.7 \mathrm{in}_{B}^{i}(P[\bar{N} / \bar{x}])$ is stable.

- $M \equiv\left\langle M_{1}, M_{2}\right\rangle$. We have $\left\langle M_{1}, M_{2}\right\rangle[\bar{N} / \bar{x}]=\left\langle M_{1}[\bar{N} / \bar{x}], M_{2}[\bar{N} / \bar{x}]\right)$. By induction hypothesis $M_{1}[\bar{N} / \bar{x}]$ and $M_{2}[\bar{N} / \bar{x}]$ are stable and by lemma $6.7\left\langle M_{1}[\bar{N} / \bar{x}], M_{2}[\bar{N} / \bar{x}]\right\rangle$ is stable.

- $M \equiv \lambda y: B . P$. Then $(\lambda y: B . P)[\bar{N} / \bar{x}]=\lambda y: B . P[\bar{N} / \bar{x}]$. Consider any stable term $R$. By inductive hypothesis $P[\bar{N} / \bar{x}][R / y]$ is stable and by lemma $6.8(\lambda y: B \cdot P[\bar{N} / \bar{x}]) R$ is stable. By definition of stability $(\lambda y: B . P[\bar{N} / \bar{x}])$ is stable.

- $M \equiv\left(M_{1} M_{2}\right)$. We have $\left(M_{1} M_{2}\right)[\bar{N} / \bar{x}]=\left(M_{1}[\bar{N} / \bar{x}] M_{2}[\bar{N} / \bar{x}]\right)$. By induction hypothesis $M_{1}[\bar{N} / \bar{x}]$ and $M_{2}[\bar{N} / \bar{x}]$ are stable and then by definition of stability for arrow types we conclude that $\left(M_{1}[\bar{N} / \bar{x}] M_{2}[\bar{N} / \bar{x}]\right)$ is stable.

- $M \equiv(\operatorname{rec} y: B \cdot P)^{i}$. Then $(\operatorname{rec} y: B \cdot P)^{i}[\bar{N} / \bar{x}]=(\operatorname{rec} y: B \cdot P[\bar{N} / \bar{x}])^{i}$. By induction hypothesis $P[\bar{N} / \bar{x}][R / y]$ is stable for a stable term $R$. In particular $y$ is stable and then $P[\bar{N} / \bar{x}][y / y]=$ $P[\bar{N} / \bar{x}]$ is stable.

We will now prove that $(\text { rec } y: B \cdot P[\bar{N} / \bar{x}])^{i}$ is stable by induction on $i$.

$-i=0$. Since $P[\bar{N} / \bar{x}]$ is stable, this comes from lemma 6.7 .

$-i>0$. Due to lemma 6.9 , it suffices to show that $P[\bar{N} / \bar{x}]\left[(\operatorname{rec} y: B \cdot P[\bar{N} / \bar{x}])^{i-1} / y\right]$ is stable. But we know by the inductive hypothesis on $i$ that $(\text { rec } y: B \cdot P[\bar{N} / \bar{x}])^{i-1}$ is stable, and we can then conclude by the inductive hypothesis on the structure.

$\square$

Corollary 6.11 Every term is stable, and hence strongly normalizing.

\section{Strong Normalization via reducibility}

In this section we will prove the strong normalization property for our calculus $\lambda_{\pi * \mu \sigma}$, with labeled recursion, but no expansions, using the reducibility method as in [GLT90], with an additional astute twist to take care of the sum type and labeled recursion. 


\subsection{Reducibility}

We define the set $R E D_{A}$ of reducible terms of type $\mathrm{A}$ by induction on the type $\mathrm{A}$ as follows:

- For $M$ of atomic type A, $M \in R E D_{A}$ iff $M$ is strongly normalizable

- For $M$ of product type, $M \in R E D_{A_{1} \times A_{2}}$ iff $\pi_{i}(M) \in R E D_{A_{i}}$

- For $M$ of a sum type, $M \in R E D_{A_{1}+A_{2}}$ iff, for fresh variables $w_{i}: A_{i}$, we have $\operatorname{Case}\left(M, \lambda x: A_{1} \cdot\left\langle x, w_{2}\right\rangle, \lambda y: A_{2} \cdot\left\langle w_{1}, y\right)\right) \in R E D_{A_{1} \times A_{2}}$ (in the case $A_{i}$ is $\mathbf{T}$, we take * instead of $u_{i}$ )

- For $M$ of a functional type, $M \in R E D_{A_{1} \rightarrow A_{2}}$ iff for all $N \in R E D_{A_{1}},(M N) \in R E D_{A_{2}}$

Some comment on the sum type are needed here: first of all notice that the notion of reducibility is well defined: reducibility for a sum type is given in term of reducibility for a product type, which has been defined before. Secondly, notice that for all other types, reducibility is either given directly as in the case of the base types, or given in terms of reducibility for types that are strictly smaller. This is not possible for the sum type, because we have no destructor associated to it, but only a case expression, so reducibility for $A+B$ really depends on reducibility of $\mathrm{A}$ and $\mathrm{B}$ together, and we express this fact by reducing it to reducibility of the product $A \times B$.

\subsection{Properties of reducibility}

Following [GLT90], we define a notion of neutrality: a term is neutral if does not interact with the surrounding context giving raise to redexes. In our case, the neutral terms are:

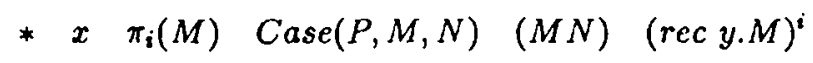

We will prove that $R E D_{A}$ enjoys the following properties, for all types $\Lambda$ :

(CR1) If $M \in R E D_{A}$, then $M$ is strongly normalizable.

(CR2) If $M \in R E D_{A}$, and $M$ reduces to $M^{\prime}$, then $M^{\prime} \in R E D_{A}$.

(CR.3) If $M$ is neutral and whenever we perform on it one step of reduction we obtain a term $M^{\prime} \in R E D_{A}$, then $M \in R E D_{A}$.

As a special case of the last clause:

(CR4) If $M$ is neutral and no reduction is applicable to it, then $M \in R E D_{A}$.

In particular, * and the variables are reducible (also the variables of type $\mathbf{T}$, as they can only reduce to $*$, which is reducible).

Proposition 7.1 (Properties of reducibility) For every type $A$, the set $R E D_{A}$ satisfies (CR1), (CR.2) and (CR3).

Proof. We will proceed by induction on the type A.

\subsubsection{Atomic types}

(CR1) A reducible term of atomic type is strongly normalizable by definition

(CR2) If $M$ is strongly normalizable, then so is every reduct of $M$ (as reduction preserves the type)

(CR3) Suppose all one step reducts of $M$ are reducible, i.e. strongly normalizable. Any reduction path leaving $M$ must pass through one of its one-step reducts, which are in a finite number, so that the longest reduction sequence starting from $M$ has length the maximum among the $1+\nu\left(M^{\prime}\right)$, as $M^{\prime}$ varies over the (one-step) reducts of $M$. Since these lengths are all finite, $M$ is strongly normalizing. 


\subsubsection{Product types}

(CR1) Suppose $M \in R E D_{A_{1} \times A_{2}}$. Then by definition we know that $\pi_{i}(M)$ are reducible and so strongly normalizing by induction hypothesis. This implies that $M$ is strongly normalizing also, because any reduction sequence starting from $M$ can be turned into a reduction sequence starting from $\pi_{i}(M)$.

(CR2) We know that $\pi_{i}(M) \in R E D_{A_{1}}$, by definition. Now consider the possible one step reducts of $M$ :

- $M$ reduces to $M^{\prime}$. Then also $\pi_{i}(M)$ reduces to $\pi_{i}\left(M^{\prime}\right)$ via the same reduction, and $M^{\prime}$ is then reducible by definition

(CR3) Let now $M$ be neutral (not necessarily reducible) such that all its one step reducts are reducible. We must show that $\pi_{i}(M)$ is reducible of type $A_{i}$. Since $M$ cannot be a pair (as it is neutral), any one step reduction of $\pi_{i}(M)$ must be to a term $\pi_{i}\left(M^{\prime}\right)$, with $M^{\prime}$ one step from $M$. By (CR2), $M^{\prime}$ is reducible, and then by definition also $\pi_{i}\left(M^{\prime}\right)$ is reducible. Now, $\pi_{i}(M)$ is neutral and all its one step reducts are reducible, hence by induction hypothesis (CR.3) for $A_{i}, \pi_{i}(M)$ is reducible, hence $M$ is, by definition.

\subsubsection{Arrow types}

(CR1) Suppose $M \in R E D_{A_{1} \rightarrow A_{2}}$. Then by definition we know that $M N$ is reducible for all reducible $N$. In particular, $M x$ is reducible for a fresh variable $x$, which is reducible by induction hypothesis (CR3) for $A_{1}$, hence $M x$ is strongly normalizable. This implies that also $M$ is strongly normalizing, as all reduction sequences starting from $M$ can be performed also on $M x$.

(CR2) Let $M \in R E D_{A_{3} \rightarrow A_{2}}$ reduce to $M^{\prime}$. For all $N \in R E D_{A_{1}}$ we have $\left(M^{\prime} N\right) \in R E D_{A_{2}}$, since it is a reduct of $(M N)$, which is reducible because $M$ and $N$ are. Hence $M^{\prime}$ is reducible by definition.

(CR3) Let now $M$ be neutral (not necessarily reducible) such that all one step reductions lead to reducible terms. We show that $M N$ is reducible for all reducible $N$ by induction on $\nu(N)$, using (CR3) for $A_{2}$. Consider a one step reduction of $M N$ : since $M$ is neutral, this reduction must be either inside $M$ or inside $N$ and leads to:

- $M^{\prime} N$, with $M^{\prime}$ one step from $M$, so $M^{\prime}$ is reducible and hence $M^{\prime} N$ is

- $M N^{\prime}$, with $N^{\prime}$ one step from $N ; N^{\prime}$ is reducible by (CR2) for $A_{1}$, and $\nu\left(N^{\prime}\right)<\nu(N)$, so by induction hypothesis $M N^{\prime}$ is reducible

Hence all reductions leaving $M N$ lead to a reducible term and hence $M N$ is reducible for all reducible $N$, so that $M$ is reducible by definition.

\subsubsection{Sum types}

(CR1) Suppose $M \in R E D_{A_{1}+A_{2}}$. Then by definition $\operatorname{Case}\left(M, \lambda x .\left(x, w_{2}\right), \lambda y .\left(w_{1}, y\right)\right) \in R E D_{A_{1} \times A_{3}}$ is reducible, hence strongly normalizable, hence $M$ is strongly normalizable too.

(CR2) Suppose $M \in R E D_{A_{1}+A_{2}}$ reduces to $M^{\prime}$. Then $\operatorname{Case}\left(M, \lambda x \cdot\left\langle x, w_{2}\right\rangle, \lambda y \cdot\left\langle w_{1}, y\right\rangle\right) \in R E D_{A_{2} \times A_{2}}$ reduces to Case $\left(M^{\prime}, \lambda x \cdot\left\langle x, w_{2}\right\rangle, \lambda y \cdot\left\langle w_{1}, y\right\rangle\right)$, so that, by (CR2) for $A \times B$ which has been proved before, $\operatorname{Case}\left(M, \lambda x .\left\langle x, w_{2}\right\rangle, \lambda y \cdot\left\langle w_{1}, y\right\rangle\right) \in R E D_{A_{1} \times A_{2}}$. Hence $M^{\prime}$ is reducible too.

(CR3) Let now $M$ be neutral, and suppose all its one step reducts are reducible. We will show that $\operatorname{Case}\left(M, \lambda x .\left\langle x, w_{2}\right\rangle, \lambda y \cdot\left\langle w_{1}, y\right\rangle\right)$ (which is neutral) is reducible using (CR3) for $A_{1} \times A_{2}$, which has already been proved to hold. Consider the possible one step reducts:

- $\operatorname{Case}\left(M^{\prime}, \lambda x \cdot\left\langle x, w_{2}\right\rangle, \lambda y \cdot\left(w_{1}, y\right\rangle\right)$ with $M^{\prime}$ one step from $M:$ then $M^{\prime}$ is reducible, hence $\operatorname{Case}\left(M^{\prime}, \lambda x \cdot\left\langle x, w_{2}\right\rangle, \lambda y \cdot\left\langle w_{1}, y\right\rangle\right)$ is reducible by definition 
- there is no other one step reduct as $M$ is neutral and the terms $\lambda x .\left\langle x, w_{2}\right\rangle$ and $\lambda y \cdot\left\langle w_{1}, y\right\rangle$ are normal

\subsection{Reducibility theorem}

We are left to show a few more lemmas:

Lemma 7.2 (Pairing) Let $M_{1}: A_{1}, M_{2}: A_{2}$ be reducible terms. Then $\left\langle M_{1}, M_{2}\right\rangle \in R E D_{A_{1} \times A_{2}}$.

Proof. We need to show that $\pi_{i}\left(\left\langle M_{1}, M_{2}\right\rangle\right) \in R E D_{A_{i}}$.

Since $\pi_{i}\left(\left\langle M_{1}, M_{2}\right\rangle\right)$ is neutral, we prove the statement using (CR3): we will show that all one step reductions are reducible. We proceed by induction on the sum $\nu\left(M_{1}\right)+\nu\left(M_{2}\right)$ of the maximum reduction lengths for $M_{1}$ and $M_{2}$, (which are finite, as these terms are strongly normalizable by (CR1)).

The possible reducts are:

- $M_{i}$, which is reducible by hypothesis

- $\pi_{i}\left(\left\langle M_{1}^{\prime}, M_{2}\right\rangle\right)$ : now, $M_{1}^{\prime}$ is one step from $M_{1}$, so that $\nu\left(M_{1}^{\prime}\right)+\nu\left(M_{2}\right)<\nu\left(M_{1}\right)+\nu\left(M_{2}\right)$, and $M_{1}^{\prime}$ is reducible by (CR2), so $\pi_{i}\left(\left\langle M_{1}^{\prime}, M_{2}\right\rangle\right)$ is reducible by induction hypothesis

- $\pi_{i}\left(\left\langle M_{1}, M_{2}^{\prime}\right\rangle\right)$ : this is shown reducible as the term in the previous case

$\square$

Lemma 7.3 (Abstraction) Let $M: A_{2}$ be a term where the variable $x: A_{1}$ may occur free. If for every $N \in R E D_{A_{1}}$ we have $M[N / x] \in R E D_{A_{2}}$, then $\lambda x: A_{1} . M \in R E D_{A_{1} \rightarrow A_{2}}$.

Proof. We want to show that $(\lambda x . M) P$ is reducible for all reducible $P$. Since this term is neutral, we can prove our Lemma using (CR3). We are then left to show that all one step reducts of $(\lambda x . M) P$ are reducible if for all $N \in R E D_{A_{1}}$ we have $M[N / x] \in R E D_{A_{2}}$. Since $M=M[x / x]$ is reducible by hypothesis (as any variable is reducible), it is strongly normalizable by (CR1), and we can proceed to prove this last statement by induction on $\nu(M)+\nu(P)$. The term $(\lambda x . M) P$ converts to:

- $M[P / x]$ which is reducible by hypothesis

- $\left(\lambda x . M^{\prime}\right) P$ with $M^{\prime}$ a reduct of $M$; now, by (CR2), $M^{\prime}$ is still reducible and furthermore $\nu\left(M^{\prime}\right)+$ $\nu(P)<\nu(M)+\nu(P)$ and $M[P / x]$ reduces to $M^{\prime}[P / x]$, and this last term is also reducible, because it is a multi-step reduct of $M[P / x]$ by Lemma 4.5 . So the induction hypothesis tells us that $\left(\lambda x . M^{\prime}\right) P$ is reducible.

- $(\lambda x . M) P^{\prime}$ with $P^{\prime}$ a reduct of $P$; now, by (CR2), $P^{\prime}$ is still reducible and furthermore $\nu(M)+$ $\nu\left(P^{\prime}\right)<\nu(M)+\nu(P)$ and $M[P / x]$ reduces to $M\left[P^{\prime} / x\right]$, by Corollary 4.8 , so this last term is also reducible. The induction hypothesis tells us that $(\lambda x . M) P^{\prime}$ is reducible.

Lemma 7.4 (Injections) For all terms, $M \in R E D_{A_{1}}$ iff in ${ }_{A_{1}+A_{2}}^{i}(M) \in R E D_{A_{1}+A_{2}}$.

Proof. $(\Rightarrow)$

We must show that $\operatorname{Case}\left(i n_{A_{1}+A_{2}}^{i}(M), \lambda x \cdot\left\langle x, w_{2}\right\rangle, \lambda y \cdot\left\langle w_{1}, y\right\rangle\right)$ is reducible of type $A_{1} \times A_{2}$, i.e. that $\pi_{i}\left(\operatorname{Case}\left(i n_{A_{1}+A_{2}}^{i}(M), \lambda x .\left\langle x, w_{2}\right\rangle, \lambda y \cdot\left(w_{1}, y\right\rangle\right)\right) \in R E D_{A_{1}}$. Since $\pi_{i}\left(\operatorname{Case}\left(i n_{A_{1}+A_{2}}^{i}(M), \lambda x .\left\langle x, w_{2}\right\rangle, \lambda y \cdot\left\langle w_{1}, y\right\rangle\right)\right)$ is neutral, we will proceed using (CR3), by induction on $\nu(M)$.

Consider then all its one step reducts:

- $\left(\left(\lambda x .\left(x, w_{2}\right\rangle\right) M\right)$, which is reducible because $M$ is reducible and $\lambda x .\left\langle x, w_{2}\right\rangle$ is reducible (by Lemma 7.2 applied to the variables $x$ and $w_{2}$ we know that $\left\langle x, w_{2}\right\rangle$ is reducible, and we get reducibility of $\lambda x \cdot\left\langle x, w_{2}\right\rangle$ by Lemma 7.3 ; similarly if we have * instead of $\left.w_{2}\right)$ 
- $\pi_{i}\left(\operatorname{Case}\left(i n_{A_{1}+A_{2}}^{i}\left(M^{\prime}\right), \lambda x \cdot\left\langle x, w_{2}\right\rangle, \lambda y \cdot\left(w_{1}, y\right\rangle\right)\right)$ with $M^{\prime}$ one step from $M$, hence reducible and $\nu\left(M^{\prime}\right)<\nu(M)$, so that it is reducible by induction hypothesis

$(\Leftrightarrow)$

Suppose now, $i n_{A_{1}+A_{2}}^{i}(M) \in R E D_{A_{1}+A_{2}}$. This means, by definition of reducibility over sum types,

$$
\operatorname{Case}\left(\operatorname{in}_{A_{1}+A_{2}}^{i}(M), \lambda x .\left\langle x, w_{2}\right\rangle, \lambda y \cdot\left\langle w_{1}, y\right\rangle\right) \in R E D_{A_{1} \times A_{2}},
$$

which implies, by definition of reducibility over product types,

$$
\pi_{1}\left(\operatorname{Case}\left(i n_{A_{1}+A_{2}}^{i}(M), \lambda x \cdot\left\langle x, w_{2}\right\rangle, \lambda y \cdot\left(w_{1}, y\right\rangle\right)\right) \in R E D_{A_{1}}
$$

This term reduces to $\pi_{1}\left(\left(\lambda x .\left\langle x, w_{2}\right\rangle M\right)\right)$, which in turn reduces to $\pi_{1}(\langle M, w\rangle)$ and then to $M$, which is then reducible by repeated use of (CR2).

Lemma 7.5 (Sum) Let $P: A+B, M: A \rightarrow C$ and $N: B \rightarrow C$ be reducible terms. Then $\operatorname{Case}(P, M, N) \in$ $R E D_{C}$.

Proof. We will work by cases on C.

$\mathrm{C}$ is an atomic type We can use (CR3) for $\mathrm{C}$, as $\operatorname{Case}(P, M, N)$ is neutral. We will show by induction on $\nu(P)+\nu(M)+\nu(N)$ that all one step reducts of $\operatorname{Case}(P, M, N)$ are reducible. Consider the possible one step reducts:

- $\operatorname{Case}\left(P^{\prime}, M, N\right)$, or $\operatorname{Case}\left(P, M^{\prime}, N\right)$, or $\operatorname{Case}\left(P, M, N^{\prime}\right)$ : they are reducible by induction hypothesis as all primed terms are reducible by (CR2) on $A+B, A \rightarrow C, B \rightarrow C$, and the measure decreases strictly.

- $(R M)$ if $P \equiv i n_{A_{1}+A_{2}}^{i}(R)$ : then $R$ is also reducible by Lemma 7.4, and this term is reducible as $M$ is

$C \equiv C_{1} \times C_{2}$ We must show $\pi_{i}(\operatorname{Case}(P, M, N)) \in R E D_{C_{i}}$. Since $\pi_{i}(\operatorname{Case}(P, M, N))$ is neutral, we can use (CR3) for $C_{i}$. Since $P, M, N$, are all reducible, they are all strongly normalizable and we can proceed by induction on the measure $\nu(P)+\nu(M)+\nu(N)$. Consider the possible one step reducts:

- $\pi_{i}\left(\operatorname{Case}\left(P^{\prime}, M, N\right)\right)$ or $\pi_{i}\left(\operatorname{Case}\left(P, M^{\prime}, N\right)\right)$ or $\pi_{i}\left(\operatorname{Case}\left(P, M, N^{\prime}\right)\right)$ : they are reducible by induction hypothesis as all primed terms are reducible by (CR2) on $A+B, A \rightarrow C$, $B \rightarrow C$, and the measure decreases strictly.

- $\pi_{i}((M R))$ if $P \equiv i n_{A_{1}+A_{2}}^{i}(R)$ : then $R$ is also reducible by Lemma 7.4 , so $M R$ is reducible and $\pi_{i}((M R))$ too

$C \equiv C_{1} \rightarrow C_{2}$ We must show $\operatorname{Case}(P, M, N) Q \in R E D_{C_{2}}$ for all $Q \in R E D_{C_{1}}$. Since Case $(P, M, N) Q$ is neutral, we can use (CR3) for $C_{2}$. Since $P, M, N, Q$ are all reducible, they are all strongly normalizable and we can proceed by induction on the measure $\nu(P)+\nu(M)+\nu(N)+\nu(Q)$. Consider the possible one step reducts:

- $\operatorname{Case}\left(P^{\prime}, M, N\right) Q$, or $\operatorname{Case}\left(P, M^{\prime}, N\right) Q$, or $\operatorname{Case}\left(P, M, N^{\prime}\right) Q$, or $\operatorname{Case}(P, M, N) Q^{\prime}:$ they are reducible by induction hypothesis as all primed terms are reducible by (CR2) on $A+B$, $A \rightarrow C, B \rightarrow C$ and $C_{1}$, and the measure decreases strictly.

- $(R M) Q$ if $P \equiv i n_{A_{1}+A_{2}}^{i}(R)$ : then $R$ is also reducible by Lemma 7.4, and this term is reducible as $M$ and $Q$ are

$C \equiv C_{1}+C_{2}$ We must show $\operatorname{Case}\left(\operatorname{Case}(P, M, N), \lambda x .\left\langle x, w_{2}\right\rangle, \lambda y \cdot\left\langle w_{1}, y\right\rangle\right) \in R E D_{C_{1} \times C_{2}}$. We can use (CR3) for $C_{1} \times C_{2}$ because $\operatorname{Case}\left(\operatorname{Case}(P, M, N), \lambda x \cdot\left\langle x, w_{2}\right\rangle, \lambda y \cdot\left\langle w_{1}, y\right\rangle\right)$ is neutral. Since $P$, $M, N$, are all reducible, thcy are all strongly normalizable and we can proceed by induction on the measure $\nu(P)+\nu(M)+\nu(N)$. Consider the possible one step reducts: 
- $\operatorname{Case}\left(\operatorname{Case}\left(P^{\prime}, M, N\right), \lambda x .\left\langle x, w_{2}\right\rangle, \lambda y \cdot\left\langle w_{1}, y\right\rangle\right)$ or $\operatorname{Case}\left(\operatorname{Case}\left(P, M^{\prime}, N\right), \lambda x .\left\langle x, w_{2}\right\rangle, \lambda y .\left\langle w_{1}, y\right\rangle\right)$ or $\operatorname{Case}\left(\operatorname{Case}\left(P, M, N^{\prime}\right), \lambda x .\left\langle x, w_{2}\right\rangle, \lambda y \cdot\left\langle w_{1}, y\right\rangle\right)$ : they are reducible by induction hypothesis as all primed terms are reducible by (CR2) on $A+B, A \rightarrow C, B \rightarrow C$, and the measure decreases strictly.

- Case $\left((R M), \lambda x \cdot\left\langle x, w_{2}\right\rangle, \lambda y \cdot\left(w_{1}, y\right\rangle\right)$ if $P \equiv i n_{A_{1}+A_{2}}^{i}(R)$ : then $R$ is also reducible by Lemma 7.4, and this term is reducible by definition as $M$, hence also $(R M)$, is

We will now prove that every reducible instance of a (not necessarily reducible) term $M$ is reducible. As a consequence, all terms will be reducible.

Theorem 7.6 (Reducibility) Let $M$ be any term (not assumed to be reducible), and suppose all the free variables of $M$ are among $x_{1}, \ldots x_{n}$ of types $A_{1}, \ldots A_{n}$. If $N_{1}, \ldots N_{n}$ are reducible terms of types $A_{1}, \ldots A_{n}$, then $M[\bar{N} / \bar{x}]$ is reducible.

Proof. By induction on the structure of $M$.

1. $M$ is *. It is neutral and normal, so it is reducible.

2. $M$ is $x_{i}$ for some $i$, then $M[\bar{N} / \bar{x}]=N_{i}$ is reducible

3. $M \equiv \pi_{i}\left(M^{\prime}\right)$. By induction hypothesis, $M^{\prime}[\bar{N} / \bar{x}]$ is reducible, hence, by definition, $\pi_{i}\left(M^{\prime}[\bar{N} / \bar{x}]\right)=$ $\pi_{i}\left(M^{\prime}\right)[\bar{N} / \bar{x}]$ is reducible.

4. $M \equiv\left\langle M_{1}, M_{2}\right\rangle$. By induction hypothesis, the terms $M_{i}[\bar{N} / \bar{x}]$ are reducible, so we conclude that the term $\left\langle M_{1}[\bar{N} / \bar{x}], M_{2}[\bar{N} / \bar{x}]\right)=\left\langle M_{1}, M_{2}\right\rangle[\bar{N} / \bar{x}]$ is reducible.

5. $M \equiv i n_{A_{1}+A_{2}}^{i}\left(M^{\prime}\right)$. By induction hypothesis, $M^{\prime}[\bar{N} / \bar{x}]$ is reducible, hence by Lemma 7.4, $i n_{A_{1}+A_{2}}^{i}\left(M^{\prime}[\bar{N} / \bar{x}]\right)=i n_{A_{1}+A_{2}}^{i}\left(M^{\prime}\right)[\bar{N} / \bar{x}]$ is reducible.

6. $M \equiv$ Case $\left(M_{1}, M_{2}, M_{3}\right)$. By induction hypothesis, the terms $M_{i}[\bar{N} / \bar{x}]$ are reducible, hence $\operatorname{Case}\left(M_{1}[\bar{N} / \bar{x}], M_{2}[\bar{N} / \bar{x}], M_{3}[\bar{N} / \bar{x}]\right)=\operatorname{Case}\left(M_{1}, M_{2}, M_{3}\right)[\bar{N} / \bar{x}]$ is reducible.

7. $M \equiv\left(M_{1} M_{2}\right)$. By induction hypothesis, the terms $M_{\mathrm{i}}[\bar{N} / \bar{x}]$ are reducible, so we conclude that the term $\left(M_{1}[\bar{N} / \bar{x}] M_{2}[\bar{N} / \bar{x}]\right)=\left(M_{1} M_{2}\right)[\bar{N} / \bar{x}]$ is reducible.

8. $M \equiv \lambda y . M^{\prime}$. Then by induction hypothesis $M^{\prime}[\bar{N} / \bar{x}]\left[N^{\prime} / y\right]$ is reducible for all reducible terms $N^{\prime}$. By Lemma $7.3, \lambda y \cdot M^{\prime}[\bar{N} / \bar{x}]=\left(\lambda y \cdot M^{\prime}\right)[\bar{N} / \bar{x}]$ is reducible.

9. $M \equiv\left(\text { recy. } M^{\prime}\right)^{i}$. By induction hypothesis, $M^{\prime}[\bar{N} / \bar{x}]$ is reducible. We will show reducibility for $\left(\text { recy. } M^{\prime}\right)^{i}[\bar{N} / \bar{x}]$ by induction on $i+\nu\left(M^{\prime}\right)$. Since (recy. $\left.M\right)^{i}[\bar{N} / \bar{x}]$ is neutral, we will use (CR3) for the type A of (recy.M) $)^{i}$. Consider the one step reducts of $(\text { recy. } M)^{i}[\bar{N} / \bar{x}]$

- $\left(\text { recy. } M^{\prime \prime}\right)^{i}[\bar{N} / \bar{x}]$ with $M^{\prime \prime}$ one step from $M^{\prime}$. Then $M^{\prime \prime}[\bar{N} / \bar{x}]$ is reducible for all reducible $\vec{N}$, because it is a multi-step reduct of the reducible term $M^{\prime}[\bar{N} / \bar{x}]$ (Lemma 4.5). Furthermore, $i+\nu\left(M^{\prime \prime}\right)<i+\nu\left(M^{\prime}\right)$, so by induction hypothesis $\left(\text { recy. } M^{\prime \prime}[\bar{N} / \bar{x}]\right)^{i}=$ $\left(\text { recy. } M^{\prime \prime}\right)^{i}[\bar{N} / \bar{x}]$ is reducible.

- $M^{\prime}[\bar{N} / \bar{x}]\left[\left(\operatorname{rec} y \cdot M^{\prime}[\bar{N} / \bar{x}]\right)^{i-1} / y\right]$. Then $\left(\text { recy. } M^{\prime}\right)^{i-1}[\bar{N} / \bar{x}]=\left(\text { recy. } M^{\prime}[\bar{N} / \bar{x}]\right)^{i-1}$ is reducible by induction hypothesis, and this tells us that $[\bar{N} / \bar{x}]\left[\left(\text { recy. } M^{\prime}[\bar{N} / \bar{x}]\right)^{i-1} / y\right]$ is a substitution of reducible terms for a set of variables containing the free variables of $M^{\prime}$, which gives us reducibility of the term $M^{\prime}[\bar{N} / \bar{x}]\left[\left(\text { recy. } M^{\prime}[\bar{N} / \bar{x}]\right)^{i-1} / y\right]$.

Corollary 7.7 (Strong Normalization) All terms are reducible, hence strongly normalizable. 


\section{Confluence of the Full Calculus}

In this section we deduce the confluence property for the calculus with bounded recursion as well as for the version with unbounded recursion.

We can immediately deduce the confluence property for the bounded system from the weak confluence and strong normalization properties using Newman's Lemma, however, we can also provide an extremely simple and neat proof that does not need the weak confluence property for the expansionary system.

Theorem 8.1 (Confluence) The relation $\Longrightarrow$ is Church-Rosser.

Proof. Since $\Longrightarrow$ is weakly confluent by theorem 4.11 and strongly normalizing by theorem 5.14 we can conclude that it is Church Rosser by the well known Newman's lemma.

The other proof of confluence proceeds as follows.

Let $M$ be a term s.t. $P_{1}^{*} \Longleftarrow M \Longrightarrow^{*} P_{2}$. Since $\Longrightarrow$ is strongly normalizing, we can reduce the terms $P_{i}$ to their normal forms $\overline{P_{i}}$. Then we have $\overline{P_{1}}{ }^{*} \Longleftarrow M \Longrightarrow{ }^{*} \overline{P_{2}}$, and by theorem 5.13 ${\overline{P_{1}}}^{\circ}+\Longleftarrow M^{\circ} \Longrightarrow+{\overline{P_{2}}}^{\circ}$ without expansions in the reduction sequences. As the system without expansions is confluent (we showed that it is strongly normalizing, and weak confluence without expansions can be shown as easily as for the simply typed lambda calculus), we can close the internal diagram with $\overrightarrow{P_{1}} \Rightarrow{ }^{*} R^{*} \Longleftarrow \overrightarrow{P_{2}}$. Now, ${\overrightarrow{P_{i}}}^{0}=$ lemma $5.6 \overline{P_{i}}$ and therefore we con complete the proof using the reductions $P_{1} \Longrightarrow^{*} \overline{P_{1}} \Longrightarrow^{*} R^{*} \Longleftarrow \overline{P_{2}}{ }^{*} \Longleftarrow P_{2}$ (notice that $\overline{P_{1}}=R=\overline{P_{2}}$ ). The following figure shows the reduction diagram:

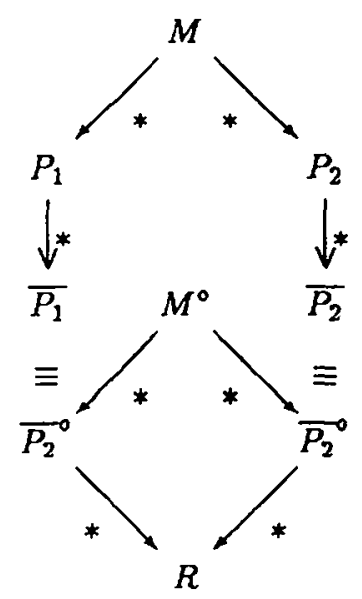

In order to show confluence of the full calculus we relate in the first place the bounded reduction $\Longrightarrow$ and the unbounded one $\stackrel{\infty}{\Longrightarrow}$, and then we use the confluence of $\Longrightarrow$ to show the confluence of $\stackrel{\infty}{\Longrightarrow}$. This very same technique, that originates from early work of Lévy [Lév76], was used in [PV87]. The connection between the reductions $\Longrightarrow$ and $\stackrel{\infty}{\Longrightarrow}$ comes from the following:

Remark 8.2 If $M \Longrightarrow^{*} N$, then $|M| \stackrel{\infty}{\Longrightarrow}|N|$, where $|M|$ is obtained from $M$ by removing all the indices from the rec terms.

Lemma 8.3 For any reduction sequence $M_{0} \stackrel{\infty}{\Longrightarrow} M_{1} \stackrel{\infty}{\Longrightarrow} \ldots \stackrel{\infty}{\Longrightarrow} M_{n}$, there exists an indexed computation $N_{0} \Longrightarrow N_{1} \Longrightarrow \ldots \Longrightarrow N_{n}$ such that $\left|N_{i}\right|=M_{i}$, for $i=0 \ldots n$.

Proof. Index all the rec constructors in $M_{0}$ by a number $n+k$, with $k \geq 0$.

Confluence of the full calculus results now from the confluence of the bounded calculus.

Theorem $8.4 \stackrel{\infty}{\Longrightarrow}$ is Church Rosser. 
Proof. Let $M \equiv P_{0} \stackrel{\infty}{\Longrightarrow} P_{1} \stackrel{\infty}{\Longrightarrow} \ldots \stackrel{\infty}{\Longrightarrow} P_{n}$ and $M \equiv Q_{0} \stackrel{\infty}{\Longrightarrow} Q_{1} \stackrel{\infty}{\Longrightarrow} \ldots \stackrel{\infty}{\Longrightarrow} Q_{m}$.

By lemma 8.3 there are indexed computations

$P_{0}^{\prime} \Longrightarrow P_{1}^{\prime} \Longrightarrow \ldots \Longrightarrow P_{n}^{\prime}$ and $Q_{0}^{\prime} \Longrightarrow Q_{1}^{\prime} \Longrightarrow \ldots \Longrightarrow Q_{m}^{\prime}$

where $\left|P_{i}\right|=P_{i}$, for $i=0 \ldots n$ and $\left|Q_{i}^{\prime}\right|=Q_{i}$, for $i=0 \ldots m$.

We can assume that $P_{0}^{\prime} \equiv Q_{0}^{\prime}$ by indexing $P^{0}$ and $Q^{0}$ with $n+m$. As $\Longrightarrow$ is Church Rosser by theorem 8.1, there exists a term $R$ such that $P_{n}^{\prime} \Longrightarrow^{*} R$ and $Q_{m}^{\prime} \Longrightarrow^{*} R$. By $8.2 P_{n}=\left|P_{n}^{\prime}\right| \stackrel{\infty}{\Longrightarrow}|R|$ and $Q_{m}=\left|Q_{m}^{\prime}\right| \stackrel{\infty}{\Longrightarrow}|R|$.

\section{Adding weak extensionality for the sum type}

In this section we show how to apply our techniques in order to accomodate in our system the weak extensionality for the sum type, that is described by the following equality, which tells us that any term $P$ of sum type $A_{1}+A_{2}$ is definitely an injection from one of the two types $A_{i}$.

$$
\operatorname{Case}\left(P, \lambda x \cdot \operatorname{in}^{1}(x), \lambda y \cdot \operatorname{in}^{2}(y)\right)=P
$$

This is the usual equality that is found in proof theory, associated to the logical connective for disjunction (see for example [GLT90, Gir72]). We call this rule "weak" because in category theory there is another stronger kind of extensional equality associated to the sum, that is used to axiomatize the uniqueness of the sum of two arrows in the diagram for the coproduct, namely

$$
\operatorname{Case}\left(P, M \circ \lambda x \cdot i n^{1}(x), M \circ \lambda y \cdot i n^{2}(y)\right)=M P
$$

where $M \circ N$ is the usual abbreviation for the composition $\lambda x .(M(N x))$.

One can easily see that this strong rule really breaks down into two simpler rules: the weak rule 1 we just introduced and the following commutation rule:

$$
\operatorname{Case}\left(P, M \circ N_{1}, M \circ N_{2}\right)=M \operatorname{Case}\left(P, N_{1}, N_{2}\right)
$$

If one really wants the equality 2 , it seems to be a difficult task to provide a confluent system for the extensional theory with arrow, product and coproduct types, as discussed in [Dou90], and to the author's best knowledge, there are no positive results in that direction.

Notice also that the equation 1 can be easily added to a reduction system with no $\mathbf{T}$ type, where all the extensional equalities are turned into contractions, as done for example in [Gal93]. In the presence of the $\mathbf{T}$ type, to use contraction rules one is forced to proceed along the lines of [CDC91], and to generatc an infinite set of reduction rules.

It is not obvious to add weak extensionality for the sum to our system, as the naive idea of adding the equality 1 as a contraction rule breaks confluence, as the following example shows:

$$
\begin{gathered}
C a s e\left(w, \lambda x: A \rightarrow B \cdot \operatorname{in}_{(A \rightarrow B)+C}^{1}(x), \lambda y: C \cdot \operatorname{in}_{(A \rightarrow B)+C}^{2}(y)\right) \longrightarrow w \\
\prod_{C a s e}\left(w, \lambda x: A \rightarrow B \cdot \sin _{(A \rightarrow B)+C}^{1}(\lambda z: A \cdot x z), \lambda y: C \cdot \sin _{(A \rightarrow B)+C}^{2}(y)\right)
\end{gathered}
$$

This problem comes from the fact that the term $\lambda x: A \rightarrow B \cdot i_{(A \rightarrow B)+C}^{1}(x)$ is not in normal form w.r.t. the rules $\eta, \delta$ and $T o p$. This also suggests the solution: it suffices to completely expand the terms $I N^{1}=\lambda x \cdot \operatorname{in}^{1}(x)$ and $I N^{2}=\lambda y \cdot i^{2}(y)$ w.r.t. the rules $\eta, \delta$ and $T o p$ (which we know now are strongly normalizing) before performing the contraction for the weak sum extensional equality.

So we are led to consider the contraction rule:

$$
\operatorname{Case}\left(P,\left\|I N^{1}\right\|,\left\|I N^{2}\right\|\right) \stackrel{+}{\longrightarrow}
$$

where $\|M\|$ denotes the normal form of $M$ w.r.t. $\eta, \delta$ and Top espansions.

It is now straightforward to check that the weak confluence property still holds, and one is left to check that the simulation theorem stays valid. 
For that, we have to verify that $\operatorname{Case}\left(P,\left\|I N^{1}\right\|,\left\|I N^{2}\right\|\right)^{\circ} \Longrightarrow+P^{\circ}$ without using expansion rules in the reduction sequence, and this is obtained by:

$$
\begin{aligned}
& \operatorname{Case}\left(P,\left\|I N^{1}\right\|,\left\|I N^{2}\right\|\right)^{\circ}= \\
& \Delta_{A+B}^{k} \operatorname{Case}\left(P^{\circ},\left\|I N^{1}\right\|^{\circ},\left\|I N^{2}\right\|^{\circ}\right)= \\
& \operatorname{Case}\left(P^{\circ},\left\|I N^{1}\right\|^{\circ},\left\|I N^{2}\right\|^{\circ}\right)= \\
& \operatorname{Case}\left(P^{\circ},\left\|I N^{1}\right\|,\left\|I N^{2}\right\|\right) \pm \\
& P^{\circ}
\end{aligned}
$$

Notice that the rules $\eta, \delta$ and Top do not create new redexes, as shown in lemma 3.8 , so in particular $\left\|I N^{i}\right\|$ is still in normal form, and the equality $\left\|I N^{i}\right\|^{\circ}=\left\|I N^{i}\right\|$ can be obtained from lemma 5.6.

\section{Conclusion and Future Work}

We have provided a confluent rewriting system for an extensional typed $\lambda$-calculus with product, sum, terminal object and recursion, which is also strongly normalizing in case the recursion operator is bounded. There are mainly two relevant technical contributions in this paper: the weak confluence proof and the simulation theorem.

On one hand, let us remark once again that the weak confluence property for a context-sensitive reduction system is not as straightforward as for the reduction systems that are congruencies. The proof is no longer just a matter of a boring but trivial case analysis, so we had to explore and analyze here the fine structure of the reduction system, showing clearly how substitution and reduction interact in the presence of context-sensitive rules.

The simulation theorem, on the other hand, turns out to be the real key tool for this expansionary system: it allows to reduce both confluence and strong normalization properties to those for the underlying calculus without expansions, that can be proved using the standard techniques. In a sense, this is all that you really need to prove.

It is also important to remark that our techniques can be applicd to many other calculi with expansionary rules. For example, these techniques are also well-behaved in polymorphic calculi, like Girard's Systems $F$ and $F_{\omega}$, which will be the argument of forthcoming work.

\section{References}

[Aka93] Y. Akama. On mints' reductions for ccc-calculus. In Typed Lambda Calculus and Applications, number 664 in LNCS. Springer Verlag, 1993.

[Bar84] H. Barendregt. The Lambda Calculus; Its syntax and Semantics (revised edition). North Holland, 1984.

[CDC91] P.-L. Curien and R. Di Cosmo. A confluent reduction system for the $\lambda$-calculus with surjective pairing and terminal object. In Leach, Monien, and Artalejo, editors, Intern. Conf. on Automata, Languages and Programming (ICALP), number 510 in LNCS, pages 291-302. Springer-Verlag, 1991.

[CF58] H. Curry and R. Feys. Combinatory Logic, volume 1. North Holland, 1958.

[Cub92] D. Cubric. On free ccc. Distributed on the types mailing list, 1992.

[DCK93] R. Di Cosmo and D. Kesner. A confluent reduction for the extensional typed $\lambda$-calculus with pairs, sums, recursion and terminal object. In A. Lingas, editor, Intern. Conf. on Automata, Languages and Programming (ICALP), LNCS. Springer-Verlag, 1993.

[Dou90] D. J. Dougherty. Some reduction properties of a lambda calculus with coproducts and recursive types. Technical report, Wesleyan University, 1990. E-mail: ddougherty eagle. wesleyan. edu. 
[Dou93] D. J. Dougherty. Some lambda calculi with categorical sums and products. In Proc. of the Fifth International Conference on Rewriting Techniques and Applications (RTA), 1993.

[Gal93] J. Gallier. On the correspondence between proofs and $\lambda$-terms. Available by anonymous ftp from ftp.cis.upenn.edu. Author's address: jean@saul.cis.upenn.edu., January 1993.

[Gir72] J.-Y. Girard. Interprétation fonctionelle et élimination des coupures dans l'arithmétique d'ordre supérieure. Thèse de doctorat d'état, Université de Paris VII, 1972.

[GLT90] J.-Y. Girard, Y. Lafont, and P. Taylor. Proofs and Types. Cambridge University Press, 1990.

[Hue76] G. Huet. Résolution d'équations dans les langages d'ordre $1,2, \ldots, \omega$. Thèse d'Etat, Université Paris VII, 1976.

[Jay92] C. B. Jay. Long $\beta \eta$ normal forms and confluence (and its revised version). Technical Report ECS-LFCS-91-183, LFCS, University of Edimburgh, 1992.

[JG92] C. B. Jay and N. Ghani. The virtues of eta-expansion. Technical Report ECS-LFCS-92-243, LFCS, University of Edimburgh, 1992.

[Klo80] J. W. Klop. Combinatory reduction systems. Mathematical Center Tracts, 27, 1980.

[Kri90] J.-L. Krivine. Lambda calculus. Types et Modéles. Masson, 1990.

[Lév76] J.-J. Lévy. An algebraic interpretation of the $\lambda \beta \kappa$-calculus and a labelled $\lambda$-calculus. Theoretical Computer Science, 2:97-114, 1976.

[LS86] J. Lambek and P. J. Scott. An introduction to higher order categorical logic. Cambridge University Press, 1986.

[Min77] G. Mints. Closed categories and the theory of proofs. Zapiski Nauchnykh Seminarov Leningradskogo Otdeleniya Matematicheskogo Instituta im. V.A. Steklova AN SSSR, 68:83$114,1977$.

[Min79] G. Mints. Teorija categorii i teoria dokazatelstv.I. Aktualnye problemy logiki i metodologii nauky, pages 252-278, 1979 .

[Pot81] G. Pottinger. The Church Rosser Theorem for the Typed lambda-calculus with Surjective Pairing. Notre Dame Journal of Formal Logic, 22(3):264-268, 1981.

[Pra71] D. Prawitz. Ideas and results in proof theory. Proceedings of the 2nd Scandinavian Logic Symposium, pages $235-307,1971$.

[PV87] A. Poigné and J. Voss. On the implementation of abstract data types by programming language constructs. Journal of Computer and System Science, 34(2-3):340-376, April/June 1987.

[Tro86] A. S. Troelstra. Strong normalization for typed terms with surjective pairing. Notre Dame Journal of Formal Logic, 27(4), 1986. 


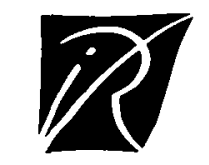

Unité de Recherche INRIA Rocquencourt

Domaine de Voluceau - Rocquencourt - B.P. 105 - 78153 LE CHESNAY Cedex (France)

Unité de Recherche INRI A Lorraine Technopôle de Nancy-Brabois - Campus Scientifique

615, rue du Jardin Botanique - B.P. 101 - 54602 VII.I.FRS LES NANCY Cedex (France)

Unité de Recherche INRIA Rennes IRISA, Campus Universitaire de Beaulieu 35042 RENNES Cedex (France)

Unité de Recherche INRIA Rhòne-AIpes 46, avenue Félix Viallet - 38031 GRENOBLE Cedex (France)

linité de Recherche INRIA Sophia Antipolis 2004, route des l.ucioles - B.P. 93 - 06902 SOPHIA ANTIPOLIS Cedex (France)

EDITEUR

INRIA - Domaine de Voluccau - Rocquencourt - B.P. 105 - 78153 LE CHESNAY Cedex (France)

ISSN $0249-6399$ 